\title{
Systematic Assessment of Neutron and Gamma Backgrounds Relevant to Operational Modeling and Detection Technology Implementation
}

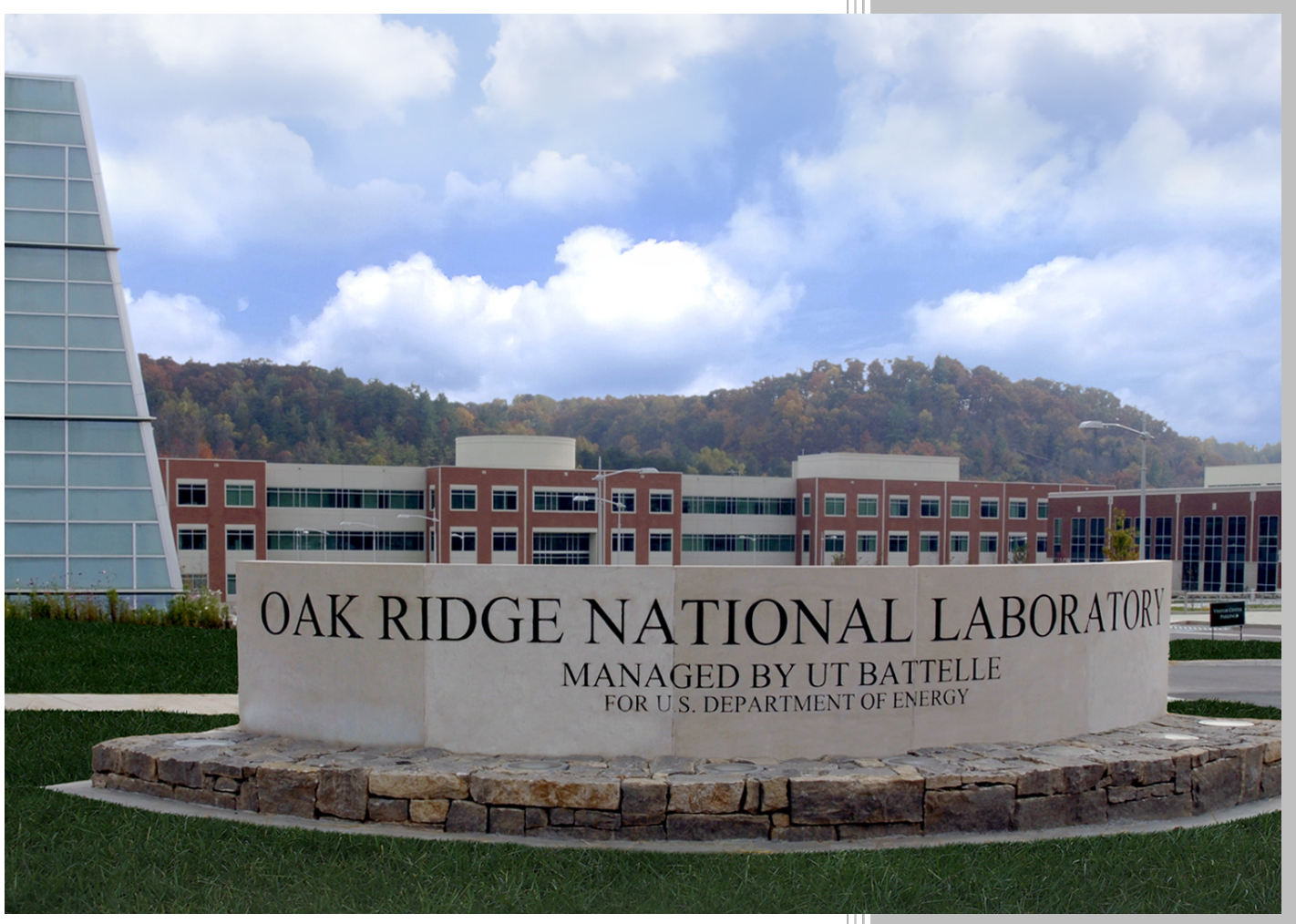

Daniel E. Archer Donald E. Hornback Jeffrey O. Johnson Thomas M. Miller Andrew D. Nicholson Bruce W. Patton Douglas E. Peplow Birsen Ayaz-Maierhafer January 2015 


\title{
DOCUMENT AVAILABILITY
}

Reports produced after January 1, 1996, are generally available free via US Department of Energy (DOE) SciTech Connect.

Website: http://www.osti.gov/scitech/

Reports produced before January 1, 1996, may be purchased by members of the public from the following source:

\author{
National Technical Information Service \\ 5285 Port Royal Road \\ Springfield, VA 22161 \\ Telephone: 703-605-6000 (1-800-553-6847) \\ TDD: $703-487-4639$ \\ Fax: 703-605-6900 \\ E-mail: info@ntis.fedworld.gov \\ Website: http://www.ntis.gov/help/ordermethods.aspx
}

Reports are available to DOE employees, DOE contractors, Energy Technology Data Exchange representatives, and International Nuclear Information System representatives from the following source:

Office of Scientific and Technical Information

PO Box 62

Oak Ridge, TN 37831

Telephone: $865-576-8401$

Fax: 865-576-5728

E-mail: report@osti.gov

Website: http://www.osti.gov/contact.html

This report was prepared as an account of work sponsored by an agency of the United States Government. Neither the United States Government nor any agency thereof, nor any of their employees, makes any warranty, express or implied, or assumes any legal liability or responsibility for the accuracy, completeness, or usefulness of any information, apparatus, product, or process disclosed, or represents that its use would not infringe privately owned rights. Reference herein to any specific commercial product, process, or service by trade name, trademark, manufacturer, or otherwise, does not necessarily constitute or imply its endorsement, recommendation, or favoring by the United States Government or any agency thereof. The views and opinions of authors expressed herein do not necessarily state or reflect those of the United States Government or any agency thereof. 
Nuclear Security and Isotope Technology Division

\title{
Systematic Assessment of Neutron and Gamma Backgrounds Relevant to Operational Modeling and Detection Technology Implementation
}

\author{
Daniel E. Archer \\ Donald E. Hornback \\ Jeffrey O. Johnson \\ Thomas M. Miller \\ Andrew D. Nicholson \\ Bruce W. Patton \\ Douglas E. Peplow \\ Birsen Ayaz-Maierhafer
}

Date Published: January 2015

\author{
Prepared by \\ OAK RIDGE NATIONAL LABORATORY \\ P.O. Box 2008 \\ Oak Ridge, Tennessee 37831-6285 \\ managed by \\ UT-Battelle, LLC \\ for the \\ US DEPARTMENT OF ENERGY \\ under contract DE-AC05-00OR22725
}




\section{CONTENTS}

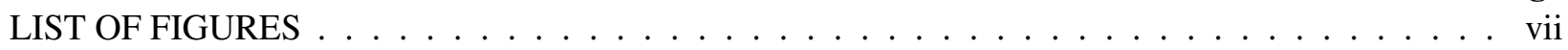

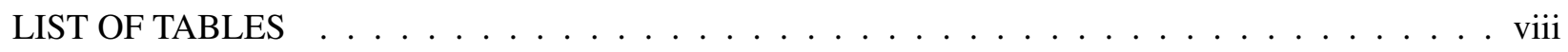

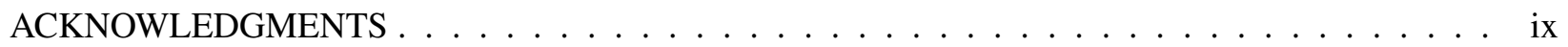

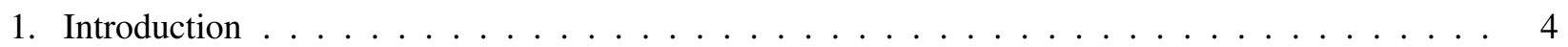

1.1 Review of findings from FY 2013 reports: Tasks 1 and $2 \ldots \ldots \ldots$. . . . . . . . 4

1.2 Discussion of the approach taken for the completion of FY 2014 Tasks 1 and 2 . . . . . . 4

1.2.1 Statement of FY 2014 Tasks . . . . . . . . . . . . . . . . . . . . . 4

1.2.2 Discussion of approach taken to complete the FY 14 Tasks . . . . . . . . . . . . 5

2. MCNP Urban Canyon Simulation Campaign . . . . . . . . . . . . . . . . . . 6

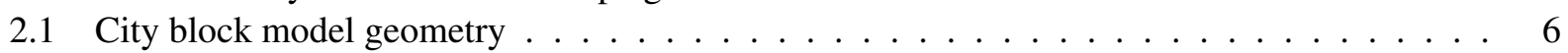

2.2 Applying hybrid calculation methods for accelerated Monte Carlo Solutions . . . . . . . . 8

2.3 Background MCNP calculations with diffuse sources . . . . . . . . . . . . . . 9

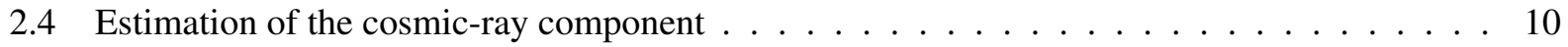

2.5 The car model and detector location . . . . . . . . . . . . . . . . . . . 10

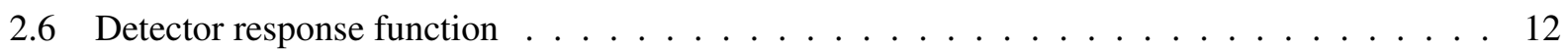

2.7 "Threat" Source (non-background) MCNP Calculations . . . . . . . . . . . . . . . . 12

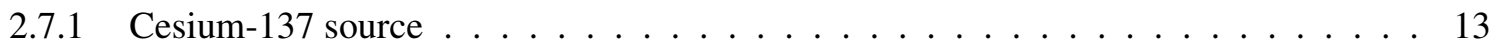

2.7.2 Highly Enriched Uranium (HEU) source . . . . . . . . . . . . . . . . . . 13

3. Simulation results and data preparation for dynamic algorithm evaluation $\ldots \ldots \ldots \ldots$

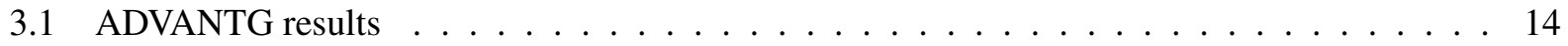

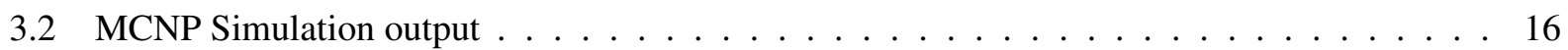

3.3 Background source component analysis . . . . . . . . . . . . . . 17

3.4 Representing dynamic conditions using sampling and interpolation . . . . . . . . . . . 17

3.5 Representative time series results with associated energy spectra . . . . . . . . . . . 18

3.6 Creation of Moving Time Windows for Synthetic Detector Output ～. . . . . . . . . . . 20

4. Analysis of Algorithm Performance on Synthetic Data _ . . . . . . . . . . . . . . . 20

4.1 Algorithm Definitions . . . . . . . . . . . . . . . . . . . . 21

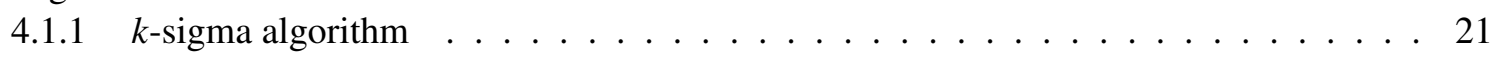

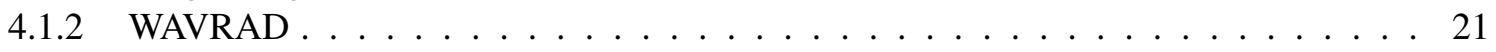

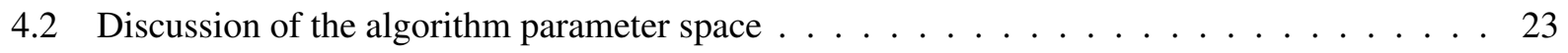

4.3 Algorithm Performance . . . . . . . . . . . . . . . . . . . . 24

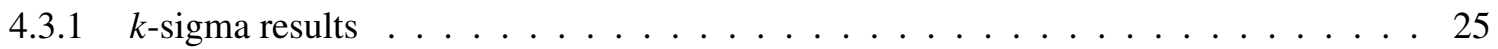

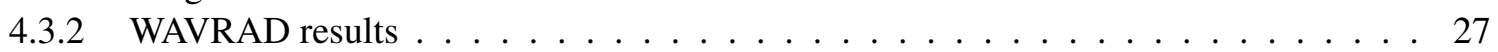

4.4 Expected performance of algorithms on real data $\ldots \ldots \ldots \ldots$

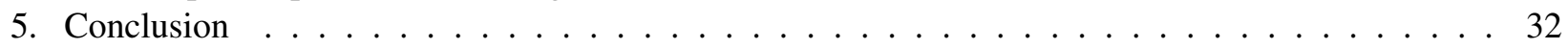

5.1 Key Findings . . . . . . . . . . . . . . . . . . . . . . . . . 32

5.2 Software Development Strategy $\ldots \ldots \ldots \ldots \ldots \ldots \ldots \ldots$

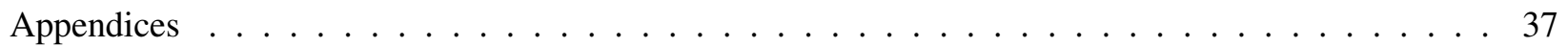

Appendix A Cosmic Ray Contributions to Photon Background in City Block Simulations . . . . . . 37

A.1 The Model, Geometry and Materials . . . . . . . . . . . . . . . . 37

A.2 The GCR Source . . . . . . . . . . . . . . . . . . . . 37

A.3 Step 1 Results and Sources for Step $2 \ldots \ldots \ldots \ldots \ldots$

A.4 Step 2 Results . . . . . . . . . . . . . . . . . . . . . . . . 43 


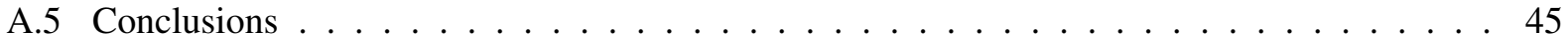

Appendix B Computational Studies with a Simplified Model of Gay Street . . . . . . . . . . . 47

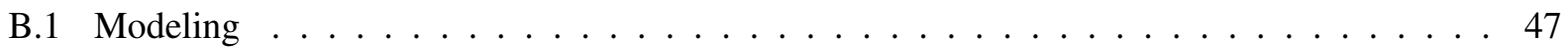

B.1.1 Geometry ........................... 47

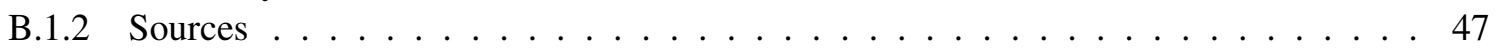

B.2 Detector Response Function $\ldots \ldots \ldots \ldots \ldots \ldots$

B.3 Source Importance Study . . . . . . . . . . . . . . . . . . . . 51

B.4 Detector Count Rate Along Gay Street Study . . . . . . . . . . . . . . . . . 53 


\section{LIST OF FIGURES}

\section{Figures}

1 Count rate vs. time for a car-mounted 2"x4"x16" NaI detector for several passes up and down Gay Street in Downtown Knoxville, Tennessee. . . . . . . . . . . . . . . . . . . 5

$23 \mathrm{D}$ view of the modeled city block highlighting the different building compositions, KUT concentrations $(\mathrm{Bq} / \mathrm{kg})$, and coordinate positions in units of meters. . . . . . . . . . . . $3 \mathrm{D}$ view of the modeled city block with labeled dimensions and building surfaces. The car model is shown with an engine block (not seen) and metal skin body (red). When source simulations are run, the source is located at the labeled location. . . . . . . . . . . . . .

4 Top-down 2D view of the center section of the modeled city block. The vehicle carrying the detector is shown along with representative individual MCNP calculation locations marked as small dots. The effective path of the vehicle is from top (+y) to bottom (-y). Details for the source indicated by the small cyan dot on the side of the road are discussed in Section 2.7. 8

5 Source probability functions for KUT as a function of energy used for background simulations. 11

6 Side view of the MCNP model used for both parked cars and the detector vehicle. . . . . . . 12

7 Comparison of MCNP detector energy spectrum to laboratory measurements of a ${ }^{137} \mathrm{Cs}$ source with a $2 " x 4 " x 16 " \mathrm{NaI}$ detector. . . . . . . . . . . . . . . . . . 13

8 HEU source term taken from GADRAS. . . . . . . . . . . . . . . . . . . . . . 14

9 Fluxes (particles/ $\mathrm{cm}^{2}$ ) from a $100 \mu \mathrm{Ci}{ }^{137} \mathrm{Cs}$ source located 0.573 meters above the street in a trash can. The source is represented by the red spot, surrounded by concentric yellow and green areas. The impact of the engine block and car shielding is demonstrated, indicating the relatively large impact that parked vehicles have in mobile detection scenarios. . . . . . .

10 Background count rates as a function of y-position in the street. The building boundaries and material activities (in $\mathrm{Bq}$ ) are denoted on the plot. . . . . . . . . . . . 16

11 Background component based simulation analyses indicate the relative importance of each background source for five different locations in the street. . . . . . . . . . . . . 17

12 Calculated background energy spectrum in a $2 " \times 4 " \times 16$ " NaI detector. The individual contributions from the decay chains of ${ }^{40} \mathrm{~K},{ }^{238} \mathrm{U},{ }^{232} \mathrm{Th}$ are shown separately, and the fractional contribution to the total count rate is shown. . . . . . . . . . . . . . . .

$13 \mathrm{MCNP}$ and interpolated background count rate as a function of discrete calculation position. The black circles represent an individual MCNP calculation position. The red points are the result of using an assumed detector speed $(4 \mathrm{~m} / \mathrm{s})$ and integration time $(0.2 \mathrm{sec})$ to interpolate between the MCNP points. . . . . . . . . . . . . . . . . . . . .

14 MCNP and sampled background spectra. (Left) A one-second sample and (right) 1000 onesecond samples averaged together. The green line represents the MCNP simulation spectrum and the blue the sampled spectrum.

15 A cartoon depiction of the data analysis of synthetic time series data. For time moving from right to left, each point represents a single position with an associated integration time and energy spectrum. Analysis integration windows of varying length for foreground (orange) and background (green) can be defined. . . . . . . . . . . . . . . . . .

16 An example plot of an interpolated count rate, foreground and background mean values, and alarm threshold for a specific detector speed and threat source strength. Since the detector moves from positive to negative relative y-coordinate (right to left), the alarm thresholds are not defined until enough points have entered into the background window (at about a relative y-coordinate of $100 \mathrm{~m}) \ldots \ldots \ldots \ldots \ldots \ldots$ 
17 An example plot from the synthetic data analysis for probability of alarm and probability of false alarm vs. a $k$-sigma value for a specific choice of FG, BG, speed, and source strength. The optimal value is found for a maximum probability of alarm for the defined tolerable

18 Probability of alarm distributions (with cars) for two different $k$-sigma choices for detection scenarios with a 1 second FG and 20 second BG. False alarm rates are denoted by horizontal dashed lines. An increase from $k=2.5$ (left plot) to $k=2.75$ (right plot) reduces the FAR to the predefined level. At the same time the probability of alarm shifts to the right, indicating reduced sensitivity to source detection. . . . . . . . . . . . . . . . . 26

19 Probability of alarm distributions for the $k$-sigma algorithm (with cars) for a ${ }^{137} \mathrm{Cs}$ spectral window 620-720 keV (left plot) and full spectrum (right plot). . . . . . . . . . . . . . 27

20 Impact of background variation on probability of detection for $25 \mathrm{~kg} \mathrm{HEU} \mathrm{(with} \mathrm{parked} \mathrm{cars).} 28$

21 WAVRAD results for ${ }^{137} \mathrm{Cs}$ for FOM's of 1.1 (top), 1.15 (middle), and 1.2 (bottom). The probability of detection values listed in Table 7 use $\mathrm{FOM}=1.15$. Solid lines are for detection without cars, while dashed lines are for calculations with cars. . . . . . . . . . . . . . 29

22 Probability of alarm for HEU as a function of speed. The blue line is for without parked cars; the dashed line is for parked cars. The reduction in the detection of the HEU for increasing speeds dramatically illustrates the increased shielding effect of parked cars. . . . . . . . . . 30

23 Count rates from RSL mobile data [12] based on the sum of four 2" $\times 4$ " $\times 16$ " NaI detectors. The dashed lines are for $k$-sigma alarms based on a $16-\sigma$ foreground deviation from the background window. Additional details are provided in the text.

A.1 GCR proton and alpha spectra during the October 1996 solar minimum $200 \mathrm{~km}$ above New York City [3] . . . . . . . . . . . . . . . . . . . . . . . . 38

A.2 City block model with parked cars and black dotted lines outlining step 1 tally surfaces and step 2 source surfaces. . . . . . . . . . . . . . . . . . . . . 39

A.3 Ground level cosmic neutron sources for city block model. . . . . . . . . . . . . . . 40

A.4 Ground level cosmic photon sources for city block model. . . . . . . . . . . . . . . . . . 40

A.5 Ground level cosmic electron sources for city block model. . . . . . . . . . . . . . . . . 41

A.6 Ground level cosmic proton sources for city block model. . . . . . . . . . . . . . . . . 41

A.7 Ground level cosmic muon sources for city block model. . . . . . . . . . . . . . . . 42

A.8 Ground level cosmic anti-muon sources for city block model. . . . . . . . . . . . . . . . 42

A.9 Total source strengths for step 2 by particle type. . . . . . . . . . . . . . . 43

A.10 Total photon count rates, by source particle type, from 50 to $3072 \mathrm{keV}$. Count rates include energy deposition by photons and electrons only. Direct energy deposition by muons and protons are not included. Indirect contributions from muons and protons are included through decay daughters, and secondary photons and electrons produced by muon and pro-

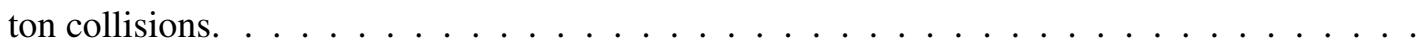

B.1 Model geometry of the 400 block of Gay Street. Materials are brick (red), granite (light gray) and concrete (dark gray) . . . . . . . . . . . . . . . . . 48

B.2 Gamma emission probabilities for the ${ }^{40} \mathrm{~K}$ source - 1Bq (0. 1344 gammas/sec). Both logarithmic scale (top) and linear scale (bottom) are shown. . . . . . . . . . . . . .

B.3 Gamma emission probabilities for the U source - 1Bq (3.4725 gammas/sec). Both logarithmic scale (top) and linear scale (bottom) are shown. . . . . . . . . . . . . . . . .

B.4 Gamma emission probabilities for the ${ }^{232} \mathrm{Th}$ source - 1Bq (3.9974 gammas/sec). Both logarithmic scale (top) and linear scale (bottom) are shown. . . . . . . . . . . . . . . . . . 
B.5 Computed detector response function ([count $\left./ \mathrm{sec}] /\left[1 / \mathrm{cm}^{2} / \mathrm{sec}\right]\right)$ for a $2 \times 4 \times 16$ inch NaI detector with a $1 \mathrm{~mm}$ thick aluminum cover $\ldots \ldots \ldots \ldots$

B.6 (Left) Urban canyon consisting of the main street, sidewalks and building faces. (Right) The canyon laid out flat $-\mathrm{a}$ basis for interpreting latter plots. . . . . . . . . . . . . . 53

B.7 Importance for a $100 \mathrm{~Bq} \mathrm{K-40} \mathrm{source} \mathrm{using} \mathrm{a} \mathrm{canyon} \mathrm{view.} \mathrm{.} \mathrm{.} \mathrm{.} \mathrm{.} \mathrm{.} \mathrm{.} \mathrm{.} \mathrm{.} \mathrm{.} \mathrm{.} \mathrm{.} \mathrm{.} \mathrm{.} \mathrm{.} \mathrm{.} \mathrm{.} 54$

B.8 Importance for a $100 \mathrm{~Bq} \mathrm{U} 238$ source using a canyon view. . . . . . . . . . . . . . . . . 55

B.9 Importance for a $100 \mathrm{~Bq}^{232} \mathrm{Th}$ source using a canyon view. . . . . . . . . . . . . . . . 56

B.10 Count rates (counts/sec) in Gay Street from ${ }^{40} \mathrm{~K}$ (left), ${ }^{238} \mathrm{U}$ (center), ${ }^{232} \mathrm{Th}$ (right). Relative uncertainties were $2 \%$ in the street . . . . . . . . . . . . . . . 57

B.11 Total detector count rates (counts/sec) from Denovo (left) and MAVRIC (middle). Relative uncertainties for MAVRIC were typically less than $1 \%$ (right) . . . . . . . . . . . 58

B.12 Count rates (counts/sec) along the center of the north-bound lane of Gay Street. The three sharp peaks correspond to the locations of the brick inlays near intersections. . . . . . . . 59

B.13 Count rate relative uncertainties along the center of the north-bound lane of Gay Street. . . . 60 


\section{LIST OF TABLES}

Tables

Page

1 KUT source ratios for model buildings and roadway . . . . . . . . . . . . . 10

2 Calculation timing study. MCNP F8 tally results (errors in parentheses) for 60 minute CPU runs. Additional details are provided in the text. . . . . . . . . . . . . . 15

3 Values for the variables used in synthetic data set generation for algorithm evaluation. . . . . 23

4 Dwell time for the modeled city street for different speeds. Since the source is placed at essentially the center of the street, the time available for background detection windows is half of the total distance transit time. . . . . . . . . . . . . . . . . . . 24

5 Foreground and background windows considered in the analysis. Combinations of foreground and background times are restricted due to a detector speed and the finite dimensions of the modeled city street. . . . . . . . . . . . . . . . . . . . . . . . 24

$6 \quad{ }^{137} \mathrm{Cs}$ source strengths (in $\mu \mathrm{Ci}$ ) required to reach a 50\% detection probability for the $k$-sigma algorithm. Data is determined from fits in Fig. 19. In the fits, $k=2.5$ was used for the ${ }^{137} \mathrm{Cs}$ spectral portion, while $k=4.0$ was used for the full spectrum. For $\mathrm{FG}=1$ second and $\mathrm{BG}=20$ seconds, speeds greater than $4 \mathrm{~m} / \mathrm{s}$ are excluded due to limited dwell time. . . . . . . . . . 26

$7 \quad{ }^{137} \mathrm{Cs}$ source strengths required to reach a $50 \%$ detection probability using the WAVRAD

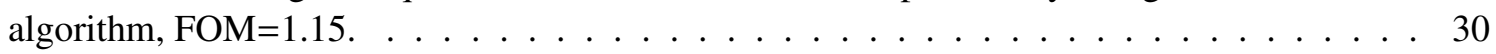

A.1 Parameters of Base Computational Model . . . . . . . . . . . . . . . 38

A.2 Directional properties of GCR induced photon background . . . . . . . . . . . 45

B.1 The buildings along Gay Street (addresses go north to south) $\ldots \ldots \ldots$. . . . . . . . 47

B.2 Data used to determine the starting masses for constructing the gamma emission spectra

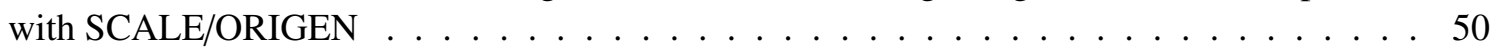

B.3 Activity densities $(\mathrm{Bq} / \mathrm{kg})$ used in the model . . . . . . . . . . . . . . . . . 50

B.4 Source strengths in the model . . . . . . . . . . . . . . . . . . 61

B.5 Computed detector response function ([count $/ \mathrm{sec}] /\left[1 / \mathrm{cm}^{2} / \mathrm{sec}\right]$ ) for a $2 \times 4 \times 16$ inch NaI detector with a $1 \mathrm{~mm}$ thick aluminum cover $\ldots \ldots \ldots \ldots$. . . . . . . . . . 62

B.6 Relative contribution to the count rate, sorted from highest contributor to lowest . . . . . . 63 


\section{ACKNOWLEDGMENTS}

- James Peltz of the National Nuclear Security Administration (NNSA) Defense Nuclear Nonproliferation (DNN) Office of Nonproliferation and Verification Research and Development (DNN R\&D) for his support and patience while serving as the program manager.

- Steven A. Schubert of Pacific Northwest National Laboratory for conducting our mid-project review with reviewers Mark S. Strickman of the U.S. Naval Research Laboratory, Prof. William S. Charlton of Texas A\&M University, and Bill Noonan of the Johns Hopkins Applied Physics Laboratory.

- Tim McCullough of RSL for enabling the use of the WAVRAD detection algorithm in this project.

- Matthew R. Kiser and colleagues at the Remote Sensing Laboratory (RSL) who supplied data.

- Daniel H. Chivers of Lawrence Berkeley National Laboratory and the University of California-Berkeley, who graciously shared data from the "Gamma-Ray Data Cloud" Project.

- Prof. Lawrence W. Townsend of the University of Tennessee (UT) for some initial guidance on space radiation codes and Wouter $\mathrm{C}$. de Wet, an undergraduate nuclear engineering student at the UT who assisted with the cosmic radiation transport calculations. 


\section{EXECUTIVE SUMMARY}

This report summarizes the findings of a two year effort to systematically assess neutron and gamma backgrounds relevant to operational modeling and detection technology implementation. The first year effort focused on reviewing the origins of background sources and their impact on measured rates in operational scenarios of interest. The second year has focused on the assessment of detector and algorithm performance as they pertain to operational requirements against the various background sources and background levels.

In the first year of effort, several operational scenarios were considered, with emphasis placed on terrestrial mobile and backpack search. High-quality data from these scenarios, defined as having associated metadata of position, weather, visual records, etc., were sought for an extensive study of background components and variability to inform operational modeling implementation. Despite a large amount of data having been collected over the past several years, the availability of high-quality data remains severely limited. This fact strongly impacted the study.

Both terrestrial and extraterrestrial sources of radiation were considered, and while extraterrestrial sources of radiation contribute to background radiation levels, it is clear that their impact to operational detector counts is essentially negligible. The well-known impact of weather on background radiation levels was studied. For instance, rain events are shown to increase gamma-ray background counts in a transitory fashion by as much as $150 \%$. At the same time, neutron count rates are strongly affected by changes in barometric pressure, with rates changing by about $30 \%$ over normal swings in pressure.

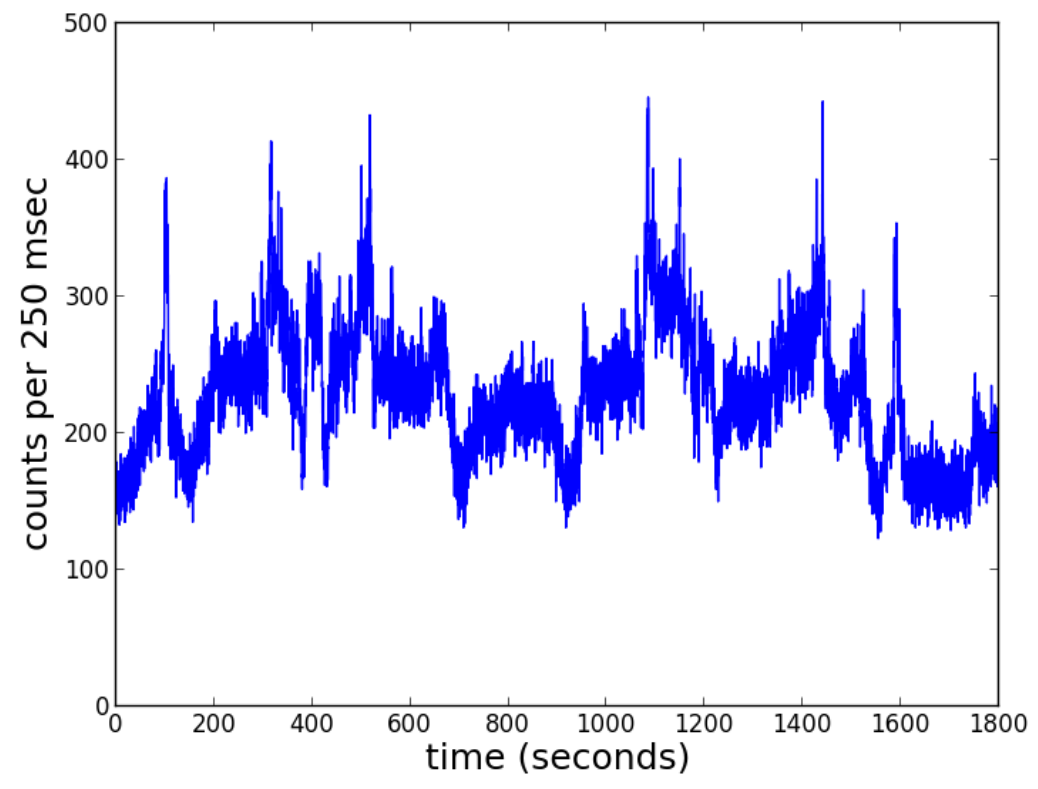

Fig. E.S.1. Count rate vs. time for a car-mounted 2"x4"x16" NaI detector for several passes up and down Gay Street in Downtown Knoxville, Tennessee.

The second year effort presented in the current report has focused on a specific detection scenario. A single detector is evaluated in an operational model of a mobile search in a city street. A realistic mixture of road surface and building materials is used to generate background gamma-ray rates. Buildings are 
assigned different materials, and thereby different background source strengths. Parked cars are included in the model to provide an additional layer of realism and complexity. Non-background threat sources are placed in this model, and a realistic detector response is implemented. From this, a synthetic detector data stream is generated consisting of a count rate and a corresponding energy spectrum for each point as the detector passes through the city street. Detection algorithms are then run against the mixture of background and threat sources, and detection probabilities are calculated. Background levels are then modified to assess the impact on detection performance.

While the desire for an operational model to provide actionable information is understandable, the complexity of the task to accurately model an urban street area cannot be overstated. Figure E.S.1 is a measured gamma-ray count rate of a standard $\mathrm{NaI}$ detector driven down a street in downtown Knoxville, TN. Peak-to-valley-fluctuations vary by more than a factor of two and presumably are attributable to the intricately changing geometries of building materials and configurations viewed by the moving detector. While capturing the full details of an actual city street in a model is prohibitive, if not impossible, the effort of implementing backgrounds in a model of this scale can be explored, and the modifications of background levels on detection probabilities can be studied. This was the focus of the effort reported in this document.

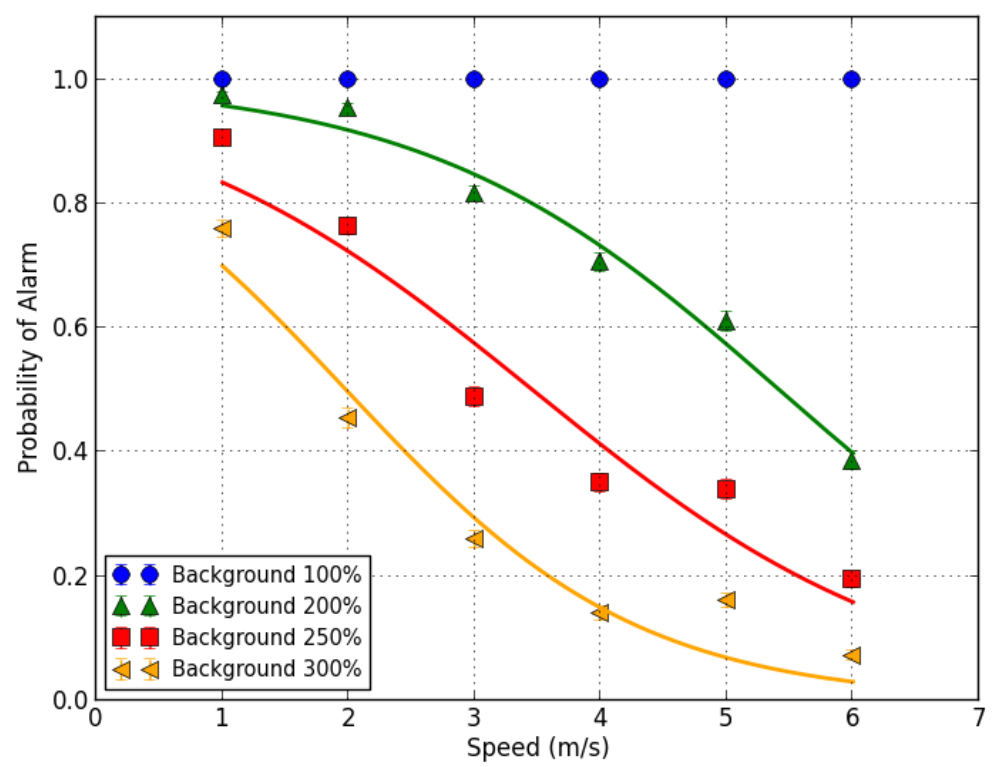

Fig. E.S.2. Impact of background variation on probability of detection for $25 \mathrm{~kg}$ highly enriched uranium (HEU) (with parked cars).

Figure E.S.2 provides a succinct summary of the results of this report. For an established background level in an operational model of a mobile search in a city street, background levels are modified and the probability of alarm is calculated as a function of speed. For relatively slow speeds $(6 \mathrm{~m} / \mathrm{s} \approx 13.4 \mathrm{mph})$, the impact on detection of doubling the background levels is dramatic, dropping the probability of alarm from $100 \%$ at $6 \mathrm{~m} / \mathrm{s}$ to less than $40 \%$. Given the large variability normally observed in measured data (as in Fig. E.S.1) which can be attributed primarily to changing background conditions, the reduction in detection probabilities associated with a doubling of the background rates is severe. It should also be noted that this reduction in detection probabilities is observed for a simple, uniform scaling of the backgrounds in the 
entire city street. A more realistic model would both scale and change background source levels and component composition with a higher spatial resolution than what was implemented in this study.

The results of this report support several key findings, some of which are already widely understood. The primary contributors to the detector background rates are those surfaces closest to the detector, which for urban search are the roadway and buildings, as well as curbs and sidewalks. Parked cars and other randomly placed objects (statues, dumpsters, etc.) have significant effects in the background rates as well as detection probabilities.

The implementation of a large operational model for this study has highlighted several key points. Model size matters, as does model detail. Significant design and implementation time for the modeling of relatively simple geometry was required. It is clear from this effort that even more modeling detail is desired, which would require significantly more effort to implement. Also, for just a few hundred meters of city block, significant computational resources were required to conduct this study. Detailed models representing larger areas of study will take a prohibitively large amount of resources to properly implement. And for the speeds associated with mobile search, a few hundred meters of city street corresponds to just a handful of seconds of dwell time.

Evaluating detection algorithms on synthetic data sets of sources with different background levels highlights the importance of background levels on detection probabilities. The modeling of background levels to the needed accuracy for a specific situation is an extremely difficult proposition. This study also highlights the sensitivity of detection to the detection parameters and details of algorithm implementation (e.g., threshold, speed, source strength, energy bins, time bins).

Based on this study, several recommendations can be made on how to proceed. From a detection perspective, there is a common sentiment that the best possible characterization of a scenario's background levels constitutes the most recent 30 seconds of (non-source) data. Regardless, more careful characterization of background levels and sources should be conducted for specific detection scenarios and locations. With recent efforts to have modelers and detector operators coordinate efforts, it could be beneficial to conduct a study that simultaneously models and measures a specific scenario with the goal of understanding the required precision and detail needed to model the observed variability in the measured data.

This project has not specifically looked at detector algorithm development, but experience in this project suggests that algorithm development may be a better way to cope with backgrounds than exquisite or exhaustive modeling techniques. For example, using pre-computed energy spectra from known background contributors in a given measurement scenario, fitting them to the measured peaks and subtracting them from the measured spectrum could help reduce the fluctuations in the important parts of the spectrum to be analyzed. Other examples could include normalizing the measured spectra to remove large changes in magnitude and then examine the spectral changes to trigger an alarm.

One of the overall results of this study is that taking a measurement and subtracting out-in real time-an estimate of background constructed from modeling is not realistic. Modeling has its place (system characterization, evaluation, constructing exercise data, etc.), but modeling the background radiation field of a specific detection scenario requires a great deal of information that cannot be collected, organized into an input and computed anywhere close to real time. Therefore, software development should focus on the detection algorithms instead of ever more precise background simulation capabilities in transport codes. 


\section{INTRODUCTION}

\subsection{REVIEW OF FINDINGS FROM FY 2013 REPORTS: TASKS 1 AND 2}

The FY 2013 Task 1 and 2 findings are briefly reviewed to provide context and motivation for the FY14 work presented in this report. A systematic study of the various sources of neutron and gamma backgrounds relevant to selected operational scenarios of interest to the end user community was conducted for FY 2013 Task 1. Static and dynamic terrestrial detection were identified as the two basic scenarios of greatest operational interest. Scenarios not considered include dynamic airborne, and static and dynamic maritime detection. For the purpose of this discussion the terms dynamic/static are defined relative to the detector (e.g., a static scenario would have a stationary detector but possibly a moving source).

The Task 1 report also provided an exhaustive review of the sources of background radiation as observed at the surface of the earth. The discussion organized the different sources of backgrounds in the following way:

Total Background $=$ Static NORM + Static Nuisance + Dynamic NORM + Dynamic Nuisance.

Static NORM (Naturally Occurring Radioactive Material) describes unchanging natural sources (e.g., terrestrial). Closely related, static nuisance sources are typically building materials (e.g., granite, street surface) that can vary according to location yet contain significant radioactive material. Dynamic NORM describes naturally occurring quantities that can change with respect to time and location (e.g., weather and cosmic rays). Dynamic nuisance sources are primarily understood to be medical isotopes. While all of these components are important to radiation detection operations, this report focuses on the static NORM and related static nuisance sources attributed to building materials. Furthermore, while background sources were studied for both gamma rays and neutrons, this report focuses on the variation of gamma ray measurements, which are identified as the more important operational detection modality.

The effects of dynamic NORM attributed to weather and cosmic rays are known to be significant and directly measurable, causing significant background gamma-ray detector count rates to increase by as much as $150 \%$ (as observed in our data analysis) for time periods of minutes and hours. Dynamic background sources are inherently more difficult to study, especially in an operational modeling environment. This project has chosen to focus on the effects of static NORM and nuisance sources that cause similar large detector count rate fluctuations over relatively small distances and detector integration times. Figure 1 is a sample plot of a $\mathrm{NaI}$ detector response driven in downtown Knoxville, TN, where the variations in count rate of more than a factor of two are due to the background changes in geography and building materials. It is this changing background environment that this report seeks to provide some insight on with regard to the contributions and importance of the background components as related to operational performance for radioactive threat detection.

\subsection{DISCUSSION OF THE APPROACH TAKEN FOR THE COMPLETION OF FY 2014 TASKS 1 AND 2}

FY 2014 Task 1 and 2 are stated, and a discussion of the approach taken for the combined report is presented.

\subsubsection{Statement of FY 2014 Tasks}

1. Task 1: Assess current detector technology spectral regions of interest and detection algorithms. Description: ORNL sends NA-22 a report describing the assessment of current detector technology 


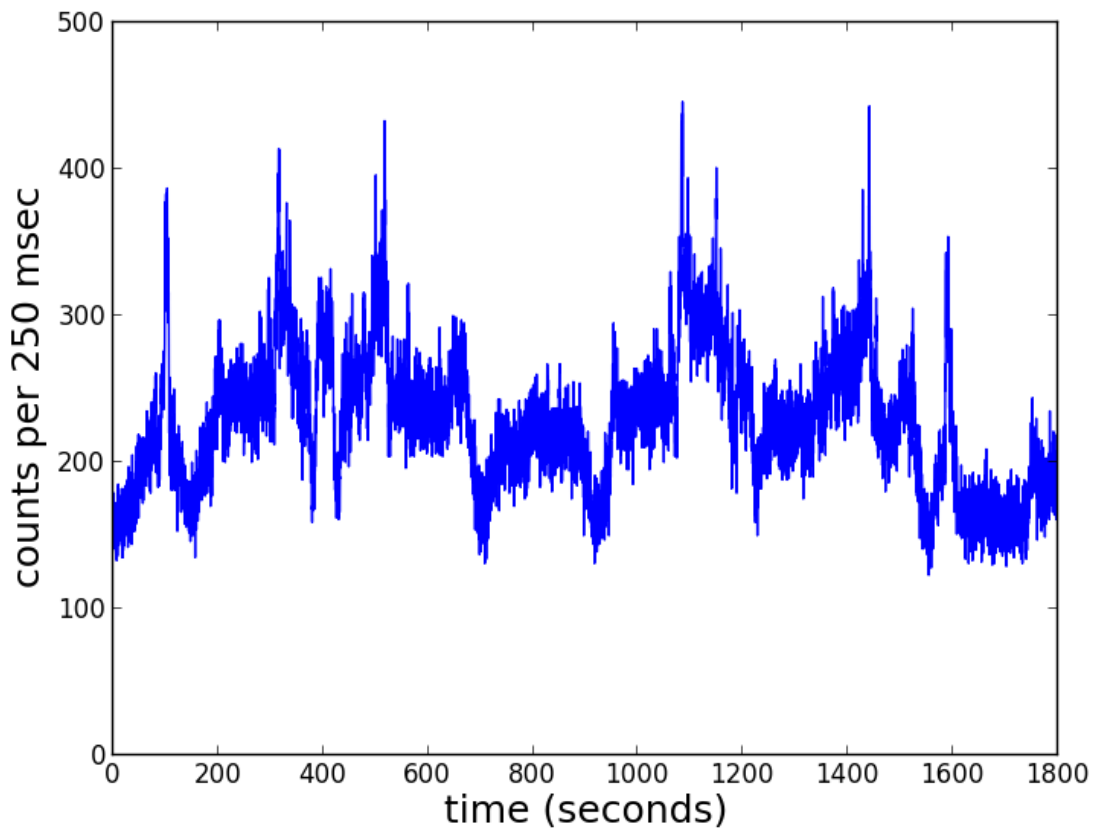

Fig. 1. Count rate vs. time for a car-mounted 2"x4"x16" NaI detector for several passes up and down Gay Street in Downtown Knoxville, Tennessee.

spectral regions of interest and detection algorithms against the magnitudes of the various sources of background as they pertain to operational requirements.

2. Task 2: Prioritize the results of the assessment into a software development strategy. Description: ORNL sends NA-22 a report describing a modeling and simulation tool development plan addressing the prioritized list of background impacts that can be reasonably addressed via improved methods development.

\subsubsection{Discussion of approach taken to complete the FY 14 Tasks}

This project studies the impact of background sources on the operational performance of detection algorithms in the following way:

1. An operational scenario is identified, and a near true-to-life model of the scenario is created. For this project an urban canyon city block was modeled in MCNP (Monte Carlo N-Particle Transport Code) [1] using a realistic scale (274 meters long, 18 meters wide, and 6.1 meter tall) and with a realistic mixture of building materials for a number of different buildings. Modeled cars are parked on the side of the street. Due to the diffuse background sources and physical scale represented, special calculation approaches were required and are discussed.

2. A mobile detector represented by a standard 2"x4"x16" NaI detector is modeled inside a car. Static simulations are performed with the detector positioned at 1 meter increments. A realistic detector response is implemented using an appropriate MCNP Gaussian energy broadening. 
3. Operationally relevant detector data are synthesized from the series of static simulations to represent a moving detector. The detector data are represented as a time series with full energy spectra available for each point.

4. A static source calculation is performed with the detector at the same positions as the background simulation points.

5. Source and background calculations are combined for different source strengths.

6. Operationally relevant algorithms are evaluated for detection performance for different source strengths and different background levels.

A real urban canyon environment is a great deal more complicated than the model described in this report, yet this simplified "real-life" model presented here affords some insight into both the challenges and complexities of operationally modeling background effects in detector performance. This effort informs the difficulties associated with operational modeling methodology.

\section{MCNP URBAN CANYON SIMULATION CAMPAIGN}

The goal of the MCNP urban canyon simulation campaign is to provide operationally relevant simulation results for a generic urban canyon environment that enables the assessment of different detection algorithm sensitivities against varying sources of background due to ${ }^{40} \mathrm{~K},{ }^{238} \mathrm{U}$, and ${ }^{232} \mathrm{Th}\left({ }^{208} \mathrm{Tl}\right)$, known collectively as KUT. This is accomplished by modeling a representative city street with realistic sources of background radiation for different buildings. Parked cars are also included to add an additional layer of realistic complexity. To represent a mobile search operation, a detector is modeled inside a car in a discrete path down one lane of the street. Separate calculations are made for the same geometry for a source located on the sidewalk at the center of the city block. All of these results based on static individual calculations are combined to produce a dynamic model of a detector moving in a complex background environment. The detector output is then analyzed using a number of known detection algorithms in order to assess their operational sensitivities against a varying background environment.

\subsection{CITY BLOCK MODEL GEOMETRY}

The MCNP city block model is based upon the dimensions of a standard New York City street. From the center of the street, these streets have two travel lanes with a width of $3.96 \mathrm{~m}(13 \mathrm{ft})$. On each side of these travel lanes is a parking lane of $2.44 \mathrm{~m}(8 \mathrm{ft})$ width, and on the outsides of the parking lanes are the sidewalks with widths of $2.44 \mathrm{~m}$ as well. The length of the city block is taken as $274 \mathrm{~m}$. In an attempt to fully characterize and describe the modeled city block, three figures are provided. Figure 2 illustrates the building dimensions (in meters) and compositions. Figure 3 provides a three-dimensional perspective looking from one end of the street. Figure 4 shows a top-down 2D view of the central $30 \mathrm{~m}$ section of the model, including the modeled vehicles and sample detector locations. The coordinate system is shown in both Fig. 3 and 4. The z-coordinate is vertical, the x-coordinate runs transverse to the street, and the $y$-coordinate runs along the street. The origin is placed at ground level at the center of the street.

The street and sidewalks are composed of concrete. The buildings facing the street are composed of concrete, upon which is placed a $0.15 \mathrm{~m}$ (6") thick covering of brick, concrete or granite as architectural enhancements. No windows were modeled for the buildings; hence the background radiation emitted from 


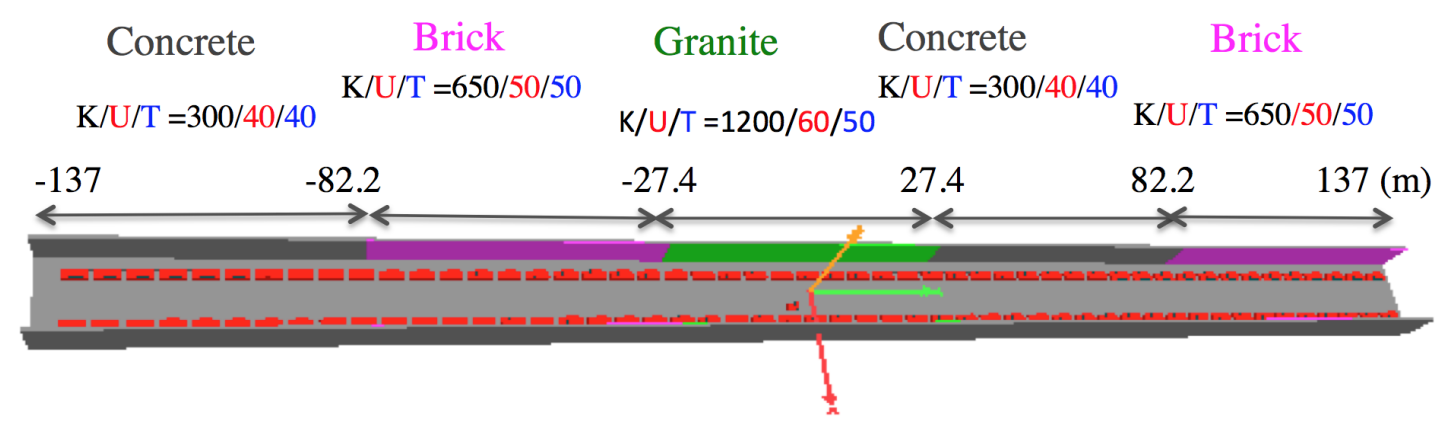

Fig. 2. 3D view of the modeled city block highlighting the different building compositions, KUT concentrations $(\mathrm{Bq} / \mathrm{kg})$, and coordinate positions in units of meters.

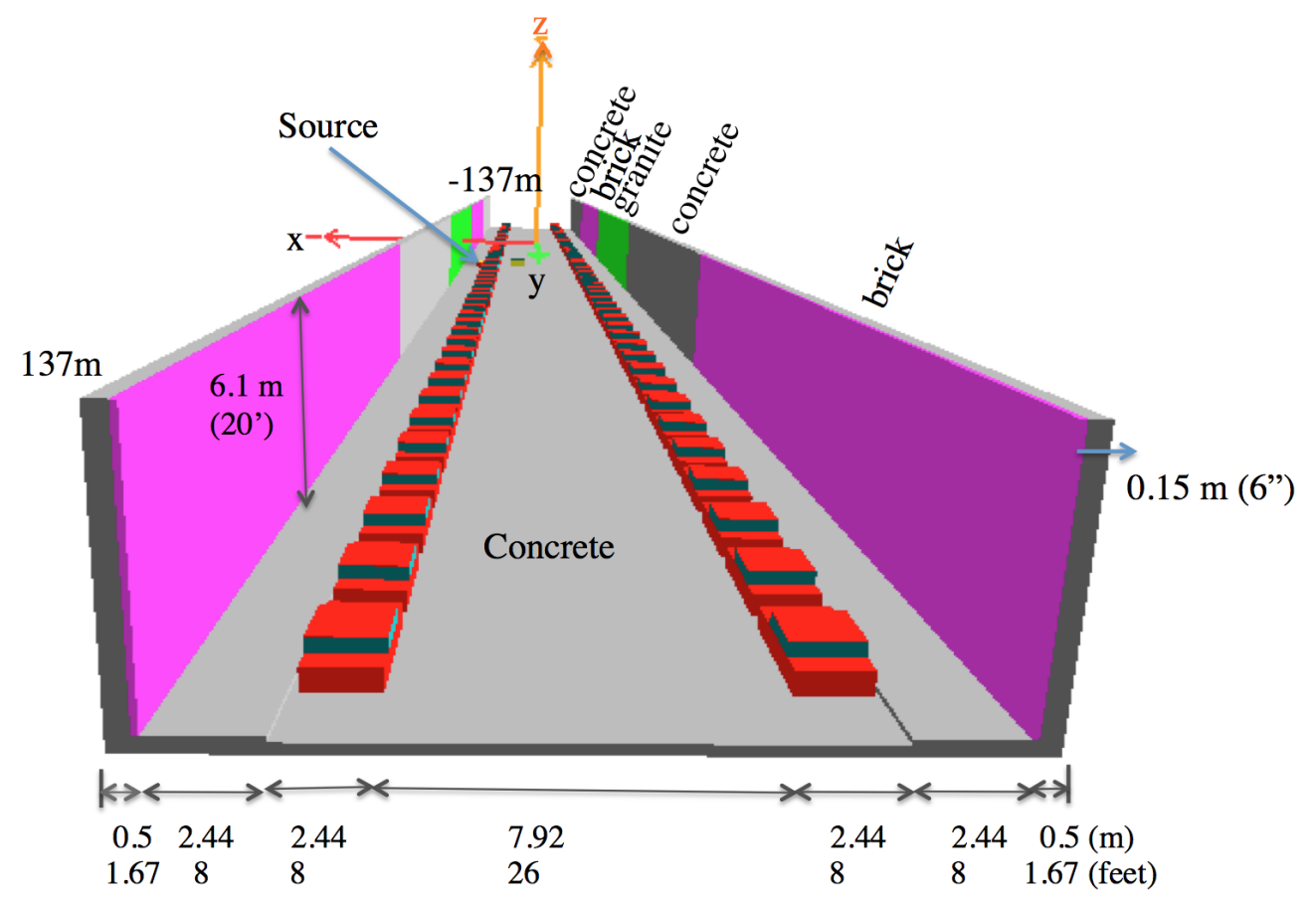

Fig. 3. 3D view of the modeled city block with labeled dimensions and building surfaces. The car model is shown with an engine block (not seen) and metal skin body (red). When source simulations are run, the source is located at the labeled location. 
the building sides will be larger than similar buildings with windows. The sidewalks are $0.15 \mathrm{~m}$ higher than the street level.

The block is divided into five segmented architectural zones each $54.8 \mathrm{~m}$ long and $6.1 \mathrm{~m}$ tall, as depicted in Fig. 3. Moving from $+y$ to $-y$, the first zone is brick, the second zone is concrete, the third (middle) zone is granite, the fourth zone is brick, and the last zone is concrete.

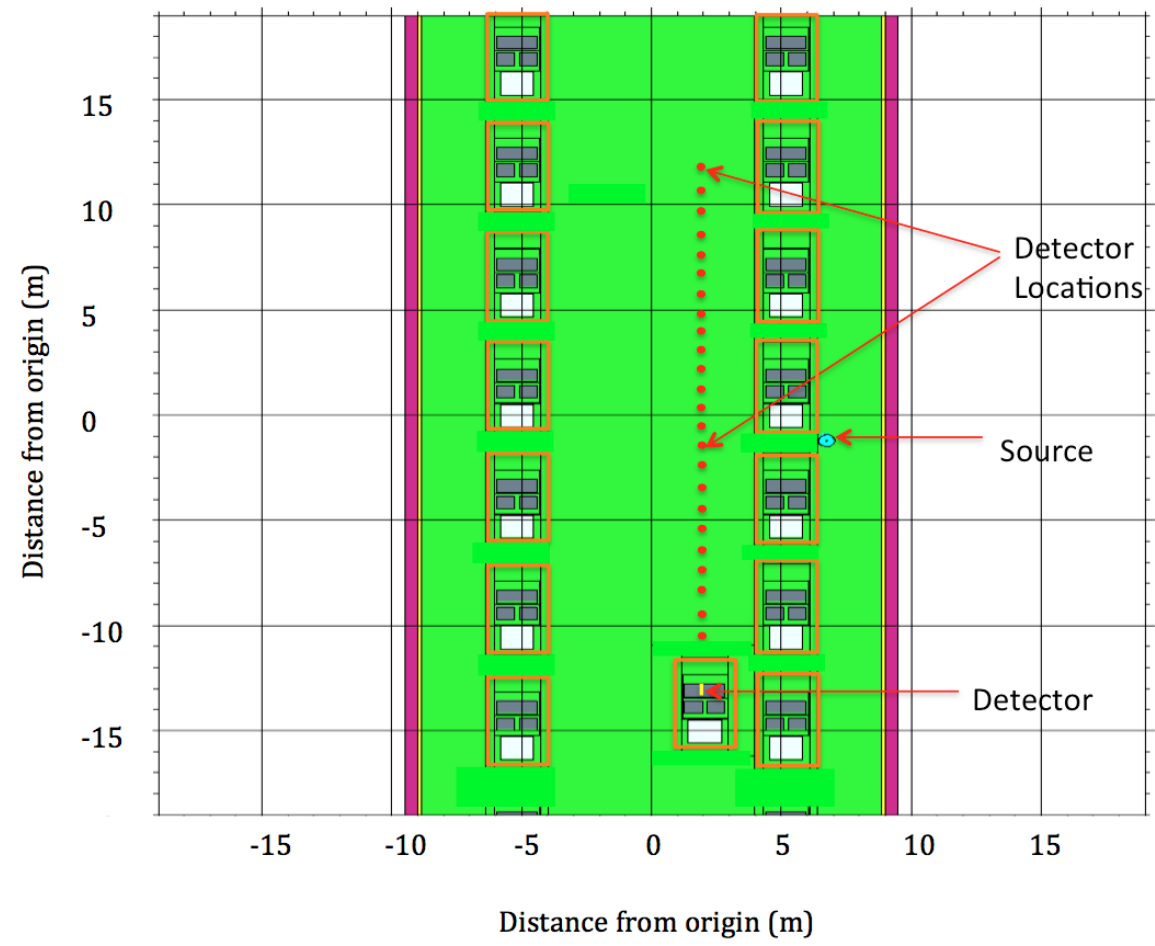

Fig. 4. Top-down 2D view of the center section of the modeled city block. The vehicle carrying the detector is shown along with representative individual MCNP calculation locations marked as small dots. The effective path of the vehicle is from top (+y) to bottom (-y). Details for the source indicated by the small cyan dot on the side of the road are discussed in Section 2.7.

\subsection{APPLYING HYBRID CALCULATION METHODS FOR ACCELERATED MONTE CARLO SOLUTIONS}

Monte Carlo particle transport calculations (such as MCNP) for deep penetration problems can require very long run times in order to achieve an acceptable level of statistical uncertainty in the final answers. Discrete ordinates codes can be faster but have limitations relative to detailed geometry modeling and large memory requirements. Monte Carlo calculations can be modified (biased) to produce results with the same variance in less time if an approximate answer or some other additional information is already known about the problem. Over time, there have been many Monte Carlo variance reduction methods developed and used. One of the most successful methods has been weight windows, which controls the weights of particles in a portion of phase space by splitting high-weight particles and rouletting low-weight particles. The difficultly with weight windows is mostly in assigning weight window values as a function of both space and energy. Another problem commonly encountered with weight windows is determining the 
source biasing properly so that source particles are not immediately split or rouletted just after birth, which could reduce the overall effectiveness of the variance reduction.

It has been recognized for a long time that the adjoint solution to a source/detector problem represents the importance of particles to contributing to that detector and that weight windows in a Monte Carlo code should be inversely proportional to the importance of a particle. Wagner and Haghighat [2] [3] developed the Consistent Adjoint Driven Importance Sampling (CADIS) method to develop weight window target values and consistent source biasing parameters which would optimize the Monte Carlo calculation for the calculation of an integrated response at a single detector location. The source used in the adjoint calculation is a detector response function at the location of the detector. Using a deterministic solution to develop the biasing parameters for the Monte Carlo calculation is now referred to as "hybrid methods." For a variety of deep penetration source/detector problems, the CADIS method can greatly reduce the Monte Carlo statistical uncertainty or required calculation time.

The CADIS method has been extended to optimize large mesh tallies by creating an importance map that converges the high-flux areas and the low-flux areas of the mesh tally at nearly the same rate, giving more uniform relative uncertainties across the mesh tally. The forward-weighted CADIS method (FW-CADIS) [4] uses the deterministic estimate of forward flux in creating the adjoint source. The adjoint source still has the energy distribution of the detector response function but has a spatial extent covering the mesh tally to be optimized, with the amount of adjoint source inversely proportional to the forward flux estimate. Like CADIS, both weight window target values and consistent source biasing parameters are produced.

The CADIS and FW-CADIS methods have been implemented into the ADVANTG package [5], which uses the Denovo discrete ordinates code to make weight window input files and source biasing cards for MCNP problems. ADVANTG has been demonstrated on many nonproliferation detection problems and has been recently integrated into the DNDO/DTRA SWORD package to accelerate MCNP calculations. ADVANTG has been well automated - requiring only a small amount of extra input with the original MCNP Monte Carlo input file.

For this work, ADVANTG and the CADIS method were used to optimize the detector response tally in each MCNP simulations. For each detector vehicle position, an importance map and source energy biasing parameters were used to increase the number of particles arriving at the detector and decrease the number of particles moving away from the detector. At each vehicle position, the calculation of the count rate in the detector from such a large distributed background source (each building face, the road, and the sidewalks), ADVANTG was essential in obtaining results with low statistical uncertainties in a reasonable number of cpu-hours. Using typical run times for ADVANTG and MCNP for a single car position, it can be shown that it takes approximately 5.3 days to do the entire block (268 car positions) for one background case using 144 Intel Xeon processors each running at $1.6 \mathrm{GHz}$. An additional calculation for just a single source adds about 1 day to the total.

\subsection{BACKGROUND MCNP CALCULATIONS WITH DIFFUSE SOURCES}

The buildings and road surfaces for the modeled urban canyon use representative KUT concentrations and are described in Table 1. The different levels of KUT for the different buildings are generated within a single MCNP calculation using geometry dependent source terms. Geometries are divided into three general categories, concrete, granite, and brick. Buildings are assigned one of these materials. The street is modeled as concrete.

Since the KUT values for building materials used in the zones can exhibit a wide variation, values for each of the architectural materials were selected based on ranges of values found in the literature and 
Table 1. KUT source ratios for model buildings and roadway

\begin{tabular}{|c|c|c|c|}
\hline \hline Building Source Material & ${ }^{40} \mathrm{~K}(\mathrm{~Bq} / \mathrm{kg})$ & Uranium $(\mathrm{Bq} / \mathrm{kg})$ & Thorium $(\mathrm{Bq} / \mathrm{kg})$ \\
\hline Concrete & 300 & 40 & 40 \\
Brick & 650 & 50 & 50 \\
Granite & 1200 & 60 & 50 \\
\hline
\end{tabular}

reported in the FY 2013 task reports [6]. The values of KUT utilized in the model are given in Table 1.

The radiation from the KUT content of NORM is due to the buildup of daughter products from long-term decay of materials from the Earth's crust. The uranium and thorium series from materials constructed from soils are in secular equilibrium. To calculate the source term for the materials in Table 1, ORIGEN [7] in the SCALE [8] package was used to decay uranium and thorium to the point of secular equilibrium. The normalized magnitude and spectra from these calculations formed the basis for the MCNP source cards. It is worth pointing out that the values in Table 1 are taken as representative values, and may or may not reflect the actual values of any particular street. The values selected were designed to give enough variation in background spectra when moving down the street to reasonably model real variations in building materials that would permit the testing of different detector algorithms.

Using the KUT values and ORIGEN calculated spectra, source energy probability distributions for concrete, granite and brick wall were calculated for use in MCNP and are shown in Fig. 5. These distributions are used for particle generation in the MCNP calculations.

The following sets of purely background calculations were performed:

1. Full city block background with parked cars

2. Full city block background without parked cars

3. Detector placed in front of single building source locations, performed separately for brick, granite, and concrete buildings. This is done with and without parked cars.

\subsection{ESTIMATION OF THE COSMIC-RAY COMPONENT}

For completeness, contributions to the gamma-ray count rate from cosmic-ray sources were considered. A conservative estimate of the galactic cosmic ray (GCR) induced photon background along the street of the city block model is approximately 20 counts/sec. Full details supporting this assertion are available in Appendix A. The measured terrestrial photon background count rates along the street of the city block model vary between 800 and 1200 counts/sec, so the estimated GCR induced photon background is less than a few percent of the modeled terrestrial photon background. Therefore, the GCR induced photon background was ignored while the various detection algorithms were tested.

\subsection{THE CAR MODEL AND DETECTOR LOCATION}

Since all of the results presented in this report are related through a detector response occurring within a modeled car, some care is now taken to describe the details of the car model. In some simulations, the parking lanes were filled with cars along each side of the street, and a single car containing the NaI detector was moved at various positions along the travel lane closest to the sidewalk containing the source. The cars are modeled rather simply, with an engine compartment, passenger compartment with seats, and a back trunk area. Figure 6 shows a side view of the model used for both the parked cars and the detector vehicle. 

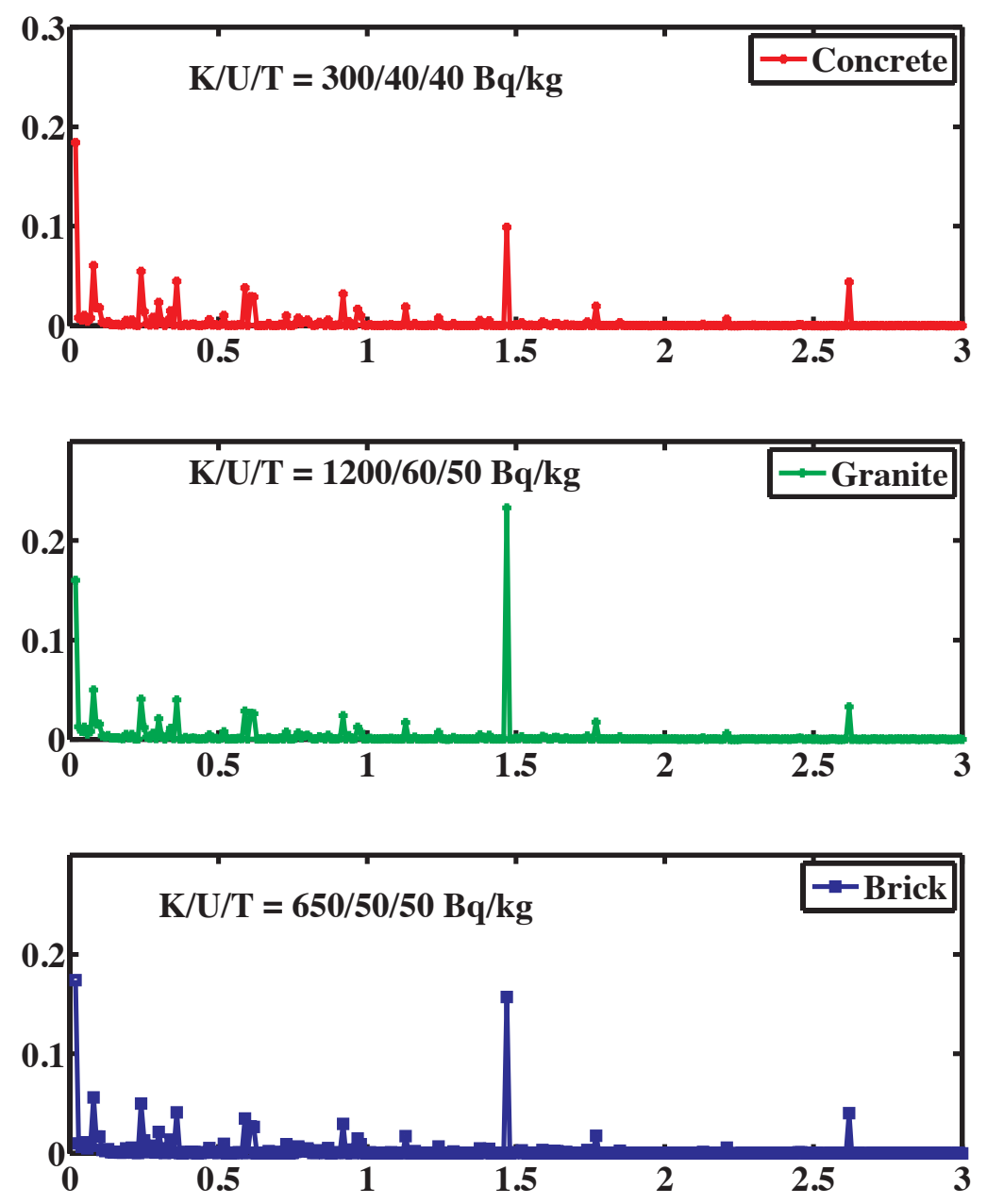

Fig. 5. Source probability functions for KUT as a function of energy used for background simulations. 
The car length is $433 \mathrm{~cm}\left(\sim 14^{\prime}\right)$ with $93.5 \mathrm{~cm}\left(\sim 3^{\prime}\right)$ of total spacing between the parked cars. A reasonable thicknesses of steel (1/16") is used for the top, bottom and sides of the car. Glass windows $(1 / 8$ ") are used for the front, back, and sides of the car. A 1/4" thick layer of cellulose was used for the car interior to mimic carpet, door padding and roof liners. The seats are also modeled using cellulose. The car engine was modeled as a 50/50 mixture of air and Fe/water/rubber combination. The tires of the vehicle were neglected. The detector is placed in the backseat of the car at window height.

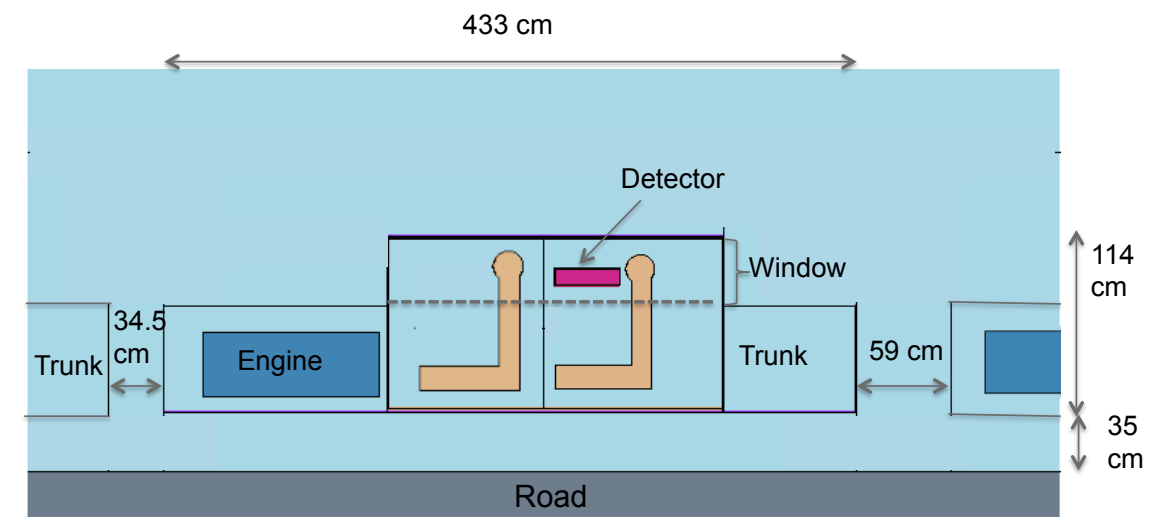

Fig. 6. Side view of the MCNP model used for both parked cars and the detector vehicle.

\subsection{DETECTOR RESPONSE FUNCTION}

Gaussian broadening is performed using the built-in MCNP Gaussian Energy Broadening with the parameters found in [9]. The resulting MCNP F8 tally for energy deposition spectrum is binned into $3 \mathrm{keV}$ wide bins. The simulated detector output was compared to an actual 2"x4"x16" laboratory measurement, as shown in Fig. 7. In order to properly reproduce the backscatter peak observed around $200 \mathrm{keV}$, the simulation geometry was closely matched to that of the laboratory. Even with the level of care taken to fully model the details of the surrounding laboratory materials, the Compton edge at $480 \mathrm{keV}$ and multiple Compton events between this edge and the $662 \mathrm{keV}$ peak are not exactly produced. Some of this sensitivity was shown to depend on the modeled choice of detector casing material and thickness. Regardless, it was determined that the modeled detector response was adequate for the purpose of this investigation.

\section{7 “THREAT"SOURCE (NON-BACKGROUND) MCNP CALCULATIONS}

Source calculations are performed with the detector in the same positions used for background calculations. The source is placed at a fixed location half-way through the city block and on the sidewalk. For source concealment, a standard city trash can (24" $(0.61 \mathrm{~m})$ OD x 33" (0.84 m) height) was modeled and filled with cellulose (density $0.25 \mathrm{~g} / \mathrm{cm}^{3}$ ) to represent paper waste. The source was placed in the middle of the trash can in both the radial and height directions. The trash can is between the bumpers of two parked cars, thus allowing a direct leakage path to the travel lane and the detector car. This will constitute the most optimistic case for detection.

Changing the source placement in the trash can is expected to affect the simulation results. A source placed at the bottom of the trash can will be more effectively shielded by surrounding cars than one placed at the top of the trash can. The effect of the source placement in a trash can packed entirely with cellulose shows about a $4 \%$ reduction in the total count rate. For the ${ }^{137} \mathrm{Cs}$ point source, the photo peak is reduced by 


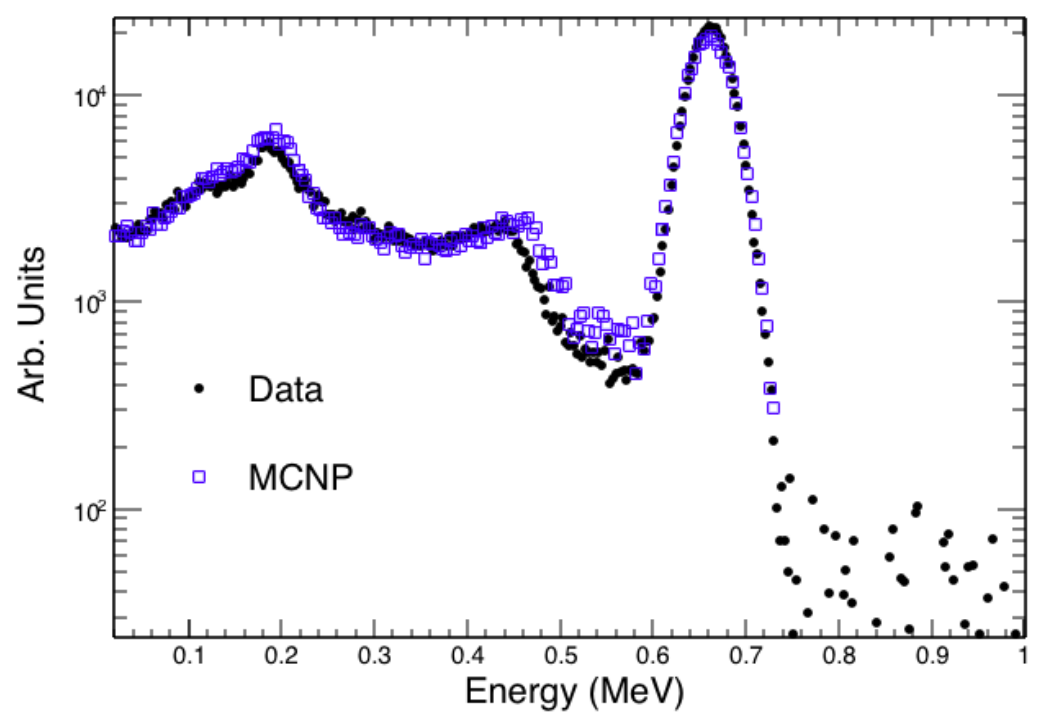

Fig. 7. Comparison of MCNP detector energy spectrum to laboratory measurements of a ${ }^{137}$ Cs source with a 2"x4"x16" NaI detector.

$\sim 50 \%$ with a commensurate increase in the down scatter continuum. All source calculation results are combined/injected into the KUT background calculation sets in order to provide a full detector response of source + background.

\subsubsection{Cesium-137 source}

The ${ }^{137} \mathrm{Cs}$ threat source MCNP calculation is performed by implementing a $662 \mathrm{keV}$ gamma source (and ignoring the associated low-energy $\mathrm{x}$-rays). The output is in units of counts/second $/{ }^{137} \mathrm{Cs}$ per $662 \mathrm{keV}$ gamma ray. As ${ }^{137} \mathrm{Cs}$ beta decays to ${ }^{137 m} \mathrm{Ba}$ with a branching ratio of $94.6 \%$, and ${ }^{137 m} \mathrm{Ba}$ decays via a 662 $\mathrm{keV}$ gamma ray with a $89.9 \%$ branching ratio, the total branching ratio for ${ }^{137} \mathrm{Cs}$ to a $662 \mathrm{keV}$ gamma ray is $0.946 \cdot 0.899=0.851$. The source simulation results are scaled by this total branching ratio to determine the amount of activity in terms of becquerels of ${ }^{137} \mathrm{Cs}$.

\subsubsection{Highly Enriched Uranium (HEU) source}

The HEU source was modeled using the gamma spectrum obtained off the outer surface of a $25 \mathrm{~kg}$ HEU sphere using GADRAS. The distribution from GADRAS using a 2"x4"x16" NaI at $8 \%$ full width half maximum (FWHM) resolution is shown in Fig. 8, and the uranium percentages are as follows: ${ }^{232} \mathrm{U}$ : $1.5 \mathrm{e}-8 \%,{ }^{234} \mathrm{U}: 0.7 \%,{ }^{235} \mathrm{U}: 93.5 \%,{ }^{236} \mathrm{U}: 0.5 \%,{ }^{238} \mathrm{U}: 5.3 \%$.

This gamma distribution was used as input into MCNP, and the HEU sphere was placed in the same location in the trash can as the original ${ }^{137} \mathrm{Cs}$ source. For the $25 \mathrm{~kg} \mathrm{HEU}$ source, the movement of the detector car was limited to $+/-10 \mathrm{~m}$ from the source location in $1 \mathrm{~m}$ increments. The calculation positions were selected so that the detector has an unobstructed view of the source trash can at the distance of closest approach. 


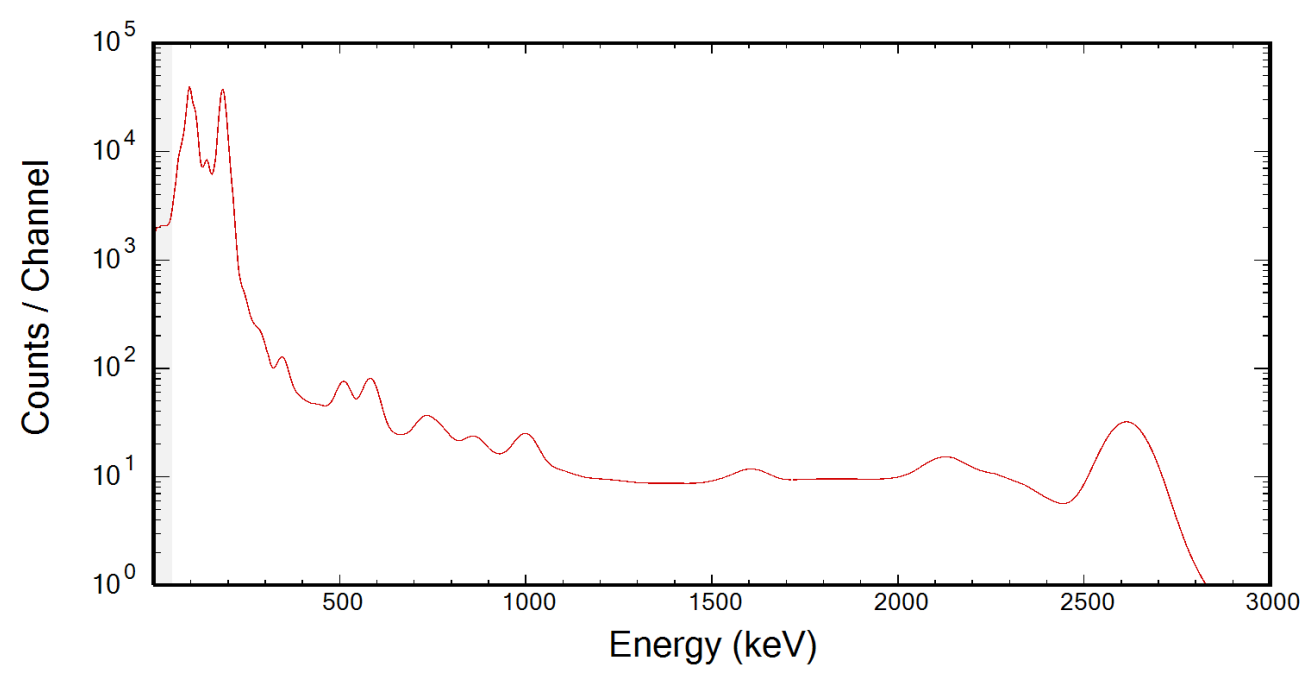

Fig. 8. HEU source term taken from GADRAS.

\section{SIMULATION RESULTS AND DATA PREPARATION FOR DYNAMIC ALGORITHM EVALUATION}

MCNP simulation results not related to algorithm evaluation are presented in this section. The series of static MCNP background calculations of a single detector positioned inside a car are performed in 1 meter increments for the entire length of the urban canyon. These simulations can be sub-sampled to approximate "dynamic" motion of the detector moving through the urban canyon.

\subsection{ADVANTG RESULTS}

In order to provide a pictorial representation of the expected radiation field in the presence of a source, ADVANTG (Section 2.2) was applied to the same street geometry used for the MCNP simulation sets. Figure 9 illustrates the importance of these leakage paths with a contour plot of the total flux from a 100 $\mu \mathrm{Ci}{ }^{137} \mathrm{Cs}$ threat source. The different colors of the contours each represent a factor of 2.5 decrease in the photon flux. The iron car engine represents the largest shielding material in the geometry. Between cars the source flux increases. Note the effectiveness of the adjacent car engines (dark blue or white rectangular areas) in reducing the flux for areas shielded by the engines. The strong leakage into the street due to the source being located between the bumpers is evident by the green contours extending into the nearest travel lane/street.

The impact of the use of ADVANTG in conjunction with MCNP was also studied. Table 2 illustrates the effect of using the ADVANTG code in conjunction with MCNP. The F8 tally values and the associated relative errors (shown in parentheses) are listed in the table. Three different detector positions were calculated: mid-point of street length $(0 \mathrm{~m})$, halfway to end of street $(66 \mathrm{~m})$, and near the end of the street $(132 \mathrm{~m})$. The MCNP CPU times were limited to 60 minutes total. The additional time for the ADVANTG calculations ranged from 16 to 24 minutes. For an F8 tally, it is generally desired to have the relative error less than 5\% (.050). Results with relative errors greater than 0.10 should be viewed as suspect in MCNP. In a Monte Carlo calculation a general rule of thumb is that it takes four times longer run times to reduce the relative error by half. Hence, for the background calculations, the analog MCNP would require four hours of total run time to get the same acceptable statistics range as the 1 hour 20 minutes MCNP-ADVANTG 


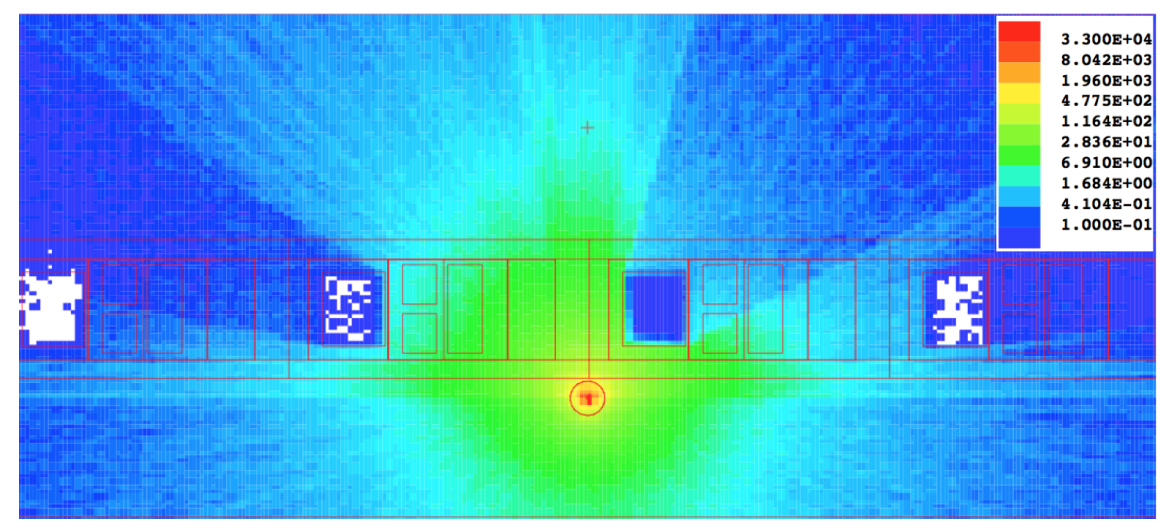

Fig. 9. Fluxes (particles/cm ${ }^{2}$ ) from a $100 \mu \mathrm{Ci}{ }^{137} \mathrm{Cs}$ source located 0.573 meters above the street in a trash can. The source is represented by the red spot, surrounded by concentric yellow and green areas. The impact of the engine block and car shielding is demonstrated, indicating the relatively large impact that parked vehicles have in mobile detection scenarios.

Table 2. Calculation timing study. MCNP F8 tally results (errors in parentheses) for 60 minute CPU runs. Additional details are provided in the text.

\begin{tabular}{|c|c|c|c|c|c|c|}
\hline \hline & \multicolumn{3}{|c|}{ Background } & \multicolumn{3}{c|}{${ }^{137}$ Cs } \\
\hline $\begin{array}{c}\text { Detector } \\
\text { Position (m) }\end{array}$ & $\begin{array}{c}\text { MCNP } \\
\text { analog }\end{array}$ & $\begin{array}{c}\text { MCNP } \\
\text { with } \\
\text { ADVANTG }\end{array}$ & $\begin{array}{c}\text { ADVANTG } \\
\text { additional } \\
\text { CPU time (min) }\end{array}$ & $\begin{array}{c}\text { MCNP } \\
\text { analog } \\
\text { with } \\
\text { ADVANTG }\end{array}$ & $\begin{array}{c}\text { ADVANTG } \\
\text { additional } \\
\text { CPU time (min) }\end{array}$ \\
\hline \hline 0 & 2213 & 2324.78 & 24.21 & $\begin{array}{c}2.48 \times 10^{-5} \\
(0.027)\end{array}$ & $\begin{array}{c}2.57 \mathrm{e}-5 \\
(0.013)\end{array}$ & 17.1 \\
& $(0.087)$ & $(0.040)$ & & $3.67 \times 10^{-8}$ & $1.84 \times 10^{-8}$ & 16.0 \\
66 & 2261.46 & 1819.82 & 24.88 & $(0.71)$ & $(0.087)$ & \\
& $(0.085)$ & $(0.041)$ & & 0.0 & $3.02 \times 10^{-9}$ & 22.2 \\
\hline 132 & 226842 & 2364.74 & 23.40 & $(0.0)$ & $(0.087)$ & \\
& $(0.085)$ & $(0.040)$ & & & & \\
\hline
\end{tabular}

run time. So, for the background case MCNP-ADVANTG converges approximately a factor of three faster than MCNP. Since, for the background case, the source (i.e., background radiation) is dispersed throughout the entire block in the building and street materials, these MCNP calculations are expected to be significantly slower than that for discrete point sources.

The same detector locations were utilized for the ${ }^{137} \mathrm{Cs}$ source located in the trash can, close to the middle of the block, offset by $-1.22 \mathrm{~m}$. Of particular interest is the improvement in answers when the detector is far from the source. Even when the detector was at $66 \mathrm{~m}$, and hence closer to the source, the analog MCNP had very poor statistics (0.71). Using our previous rule of thumb in which a four times longer run time provides half the relative error, we would have to run the analog MCNP $(0.71 \times 0.5 \times 0.5 \times$ $0.5=0.089) 64$ hours to get the same statistics as the 1 hour 16 minutes of the MCNP-ADVANTG combination. Thus, the overall speed increase is approximately a factor of 50 ( 64 hours/1.25 hours $=51.2$ ).

For the detector at $132 \mathrm{~m}$, not a single photon was tallied during a $60 \mathrm{~min}$ analog MCNP calculation, while the MCNP-ADVANTG combination obtained decent statistics in approximately 1 hour 22 min total cpu time. Therefore, it can be concluded that for these problems the speed increase due to the MCNP-ADVANTG combination ranges from a factor of three to better than 50. For the ${ }^{137}$ Cs source with 
the detector located at the extreme ends of the block, it is valid to assume that analog MCNP would never reach an acceptable answer.

\subsection{MCNP SIMULATION OUTPUT}

Several simulation sets were calculated with one meter position spacing down the length of the city block (as detailed in Fig. 4). This report includes results for the following:

1. Background without cars

2. Background with cars

3. ${ }^{137} \mathrm{Cs}$ source with cars, positioned behind a car $(-1.22 \mathrm{~m}$ from $\mathrm{y}=0)$, combined with background with cars set

4. ${ }^{137} \mathrm{Cs}$ source with cars, positioned between cars $(+1.80 \mathrm{~m}$ from $\mathrm{y}=0)$, combined with background with cars set

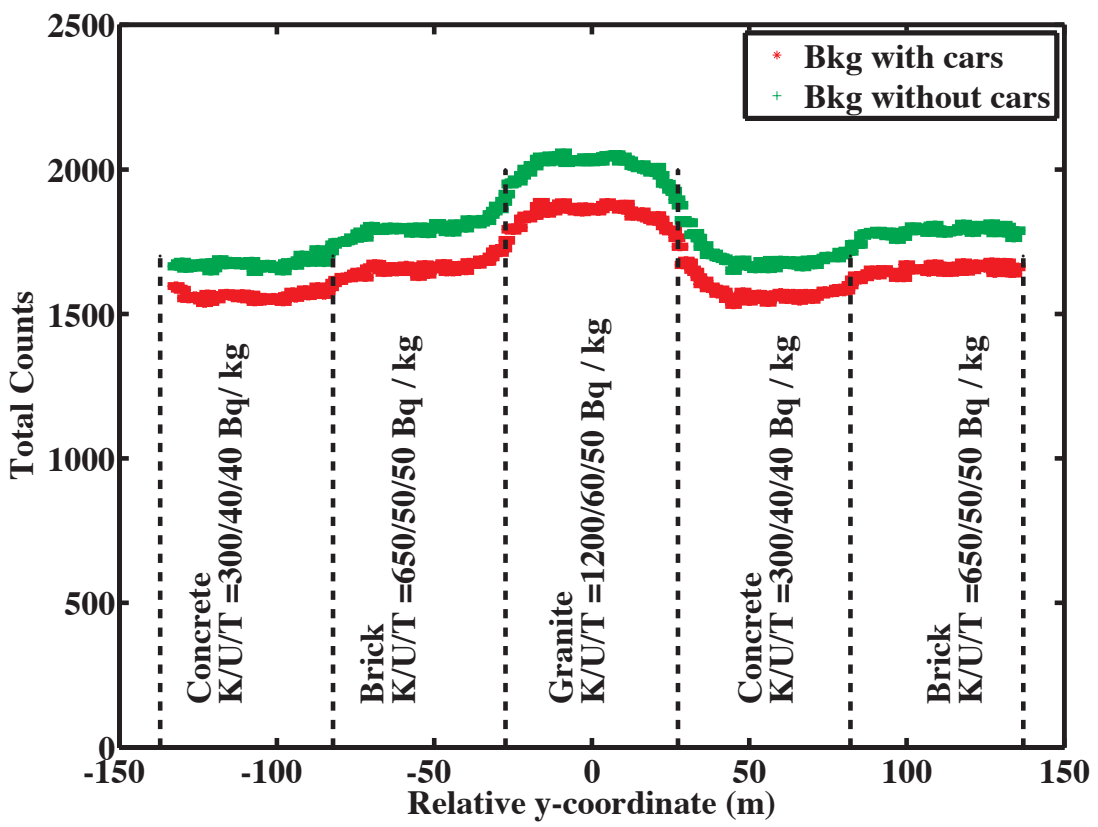

Fig. 10. Background count rates as a function of y-position in the street. The building boundaries and material activities (in $\mathrm{Bq}$ ) are denoted on the plot.

Figure 10 shows the calculated count rate as a function of position in the street using the detector response described in Section 2.6. The count rates shown in the figure are due strictly to the background radiation sources assigned according to Table 1 . Two simulation set results are shown, one with cars parked in the street and one without cars. As expected the presence of cars is shown to decrease the detected gamma count rates by about $10 \%$. The importance of the building materials is also highlighted. The building boundaries noted on the plot are clearly reflected in the related count rates. Even though the count 
rates differ by only $10 \%$, it is worth noting that for the detector moving down a traffic lane, the spectral shape will be affected by the cars, with photo peaks suppressed and a corresponding increase in the down-scatter continuum.

\subsection{BACKGROUND SOURCE COMPONENT ANALYSIS}

In order to study the importance of different background components, namely, the road surface, the sidewalks, and the different building materials, the simulated detector was placed in front of each building on the street. Separate calculations were performed in front of each building, and the source of particles reaching the detector are identified. The road surface accounts for $60-70 \%$ of the count rate, with the second largest contribution coming from the closest building. These results are shown in Fig. 11.

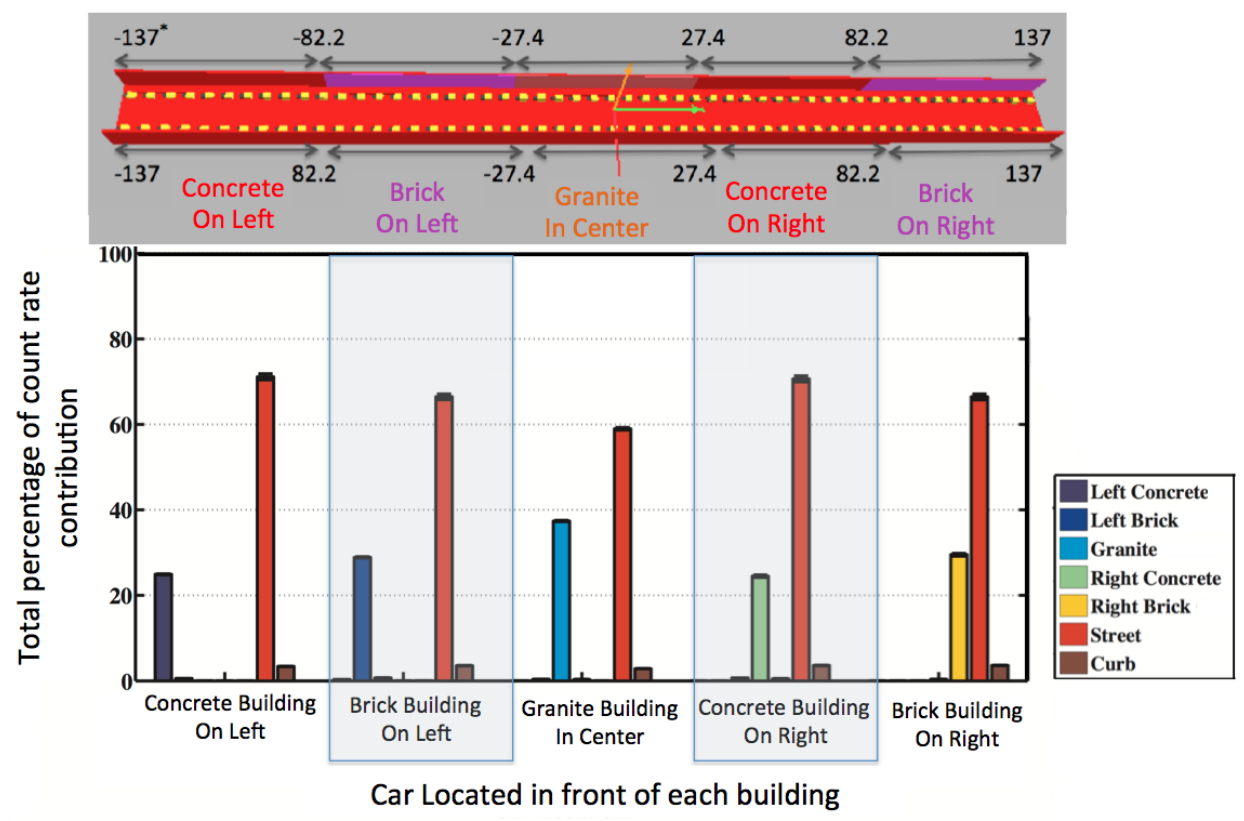

Fig. 11. Background component based simulation analyses indicate the relative importance of each background source for five different locations in the street.

Similarly, tallying separate contributions in the calculated detector response from ${ }^{40} \mathrm{~K},{ }^{238} \mathrm{U}$, and ${ }^{232} \mathrm{Th}$ shows the relative contribution in count rate and spectral features of each of the primary background components (Fig. 12).

\subsection{REPRESENTING DYNAMIC CONDITIONS USING SAMPLING AND INTERPO- LATION}

The set of static MCNP simulations performed along the detector path is used to generate detector time series output. For a stated speed, a set of evenly spaced positions are calculated using a 0.2 second detector integration time. At each position a time normalized weighted average gamma ray spectrum along with the propagated error is calculated from the two nearest MCNP simulations. From this spectrum, a total count rate and error are calculated. The total count rate and error are used to create a normal distribution, which is then sampled to arrive at a sampled total count rate. The weighted average gamma ray spectrum is scaled 


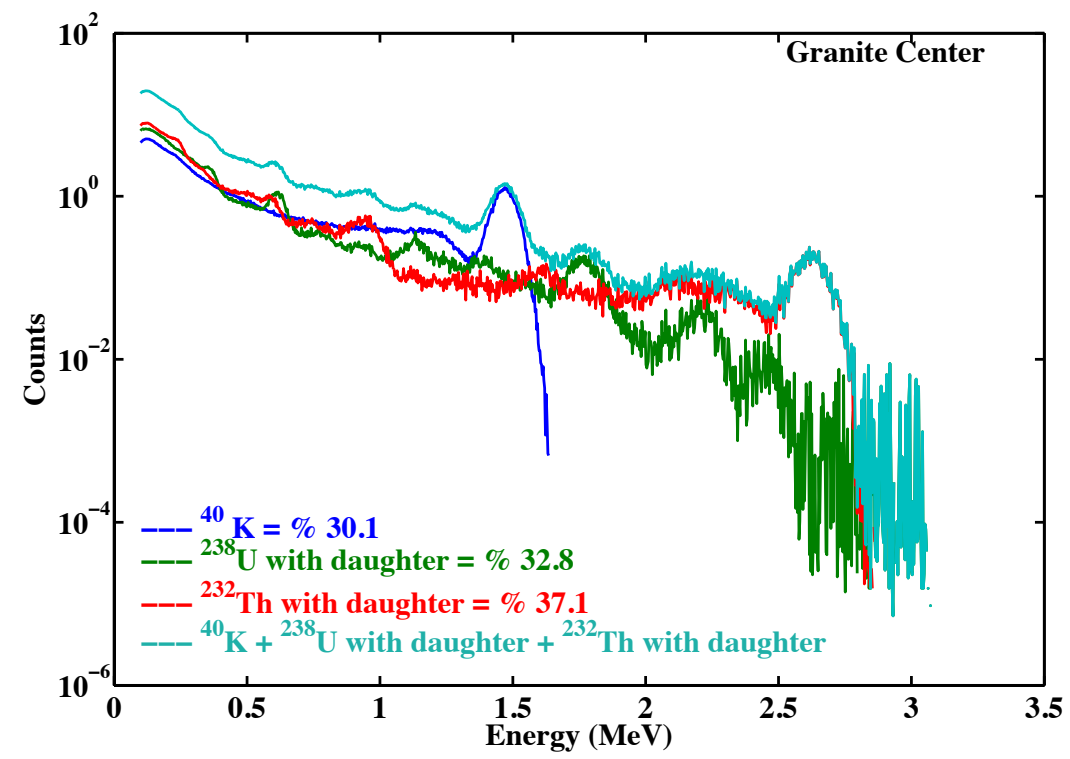

Fig. 12. Calculated background energy spectrum in a 2" $\times 4$ " $\times 16$ " NaI detector. The individual contributions from the decay chains of ${ }^{40} \mathrm{~K},{ }^{238} \mathrm{U},{ }^{232} \mathrm{Th}$ are shown separately, and the fractional contribution to the total count rate is shown.

by the ratio of the sampled total count rate and the weighted average total count rate to arrive at the interpolated gamma ray spectrum. Figure 13 shows an example interpolated background count rate as a function of street position.

This method is applied to the source and background simulations independently. Since the background MCNP simulation tally results represent a count rate, no further scaling is needed. The threat source MCNP tally results are reported on a per-particle-emitted basis, with the weights of all gamma rays equal to unity. To arrive at a source count rate, for a specified source strength, the entire source spectrum is scaled by the number of gamma rays produced per decay (including branching ratios) to arrive at a source generated count rate. At the end of the procedure, the source and background interpolations are added together to produce the total interpolated gamma ray spectra.

\subsection{REPRESENTATIVE TIME SERIES RESULTS WITH ASSOCIATED ENERGY SPEC- TRA}

For a single static calculation point, the MCNP generated spectrum represents dwell times long enough to minimize the final associated MCNP error. To create data more representative for a moving detector, each energy bin of the interpolated spectrum is sampled using Poisson statistics. Figure 14 shows a representative energy spectrum derived from neighboring static MCNP calculations. A random seed is used in the sampling so that the generated energy spectra are statistically unique each time they are generated. In this way the single set of static MCNP runs can be used to generate many different dynamic results in order to calculate both probability of detection and false alarm rates. For each source strength and detector speed combination, 1000 sets of sampled source path spectra were calculated to be used by the detection algorithms. 


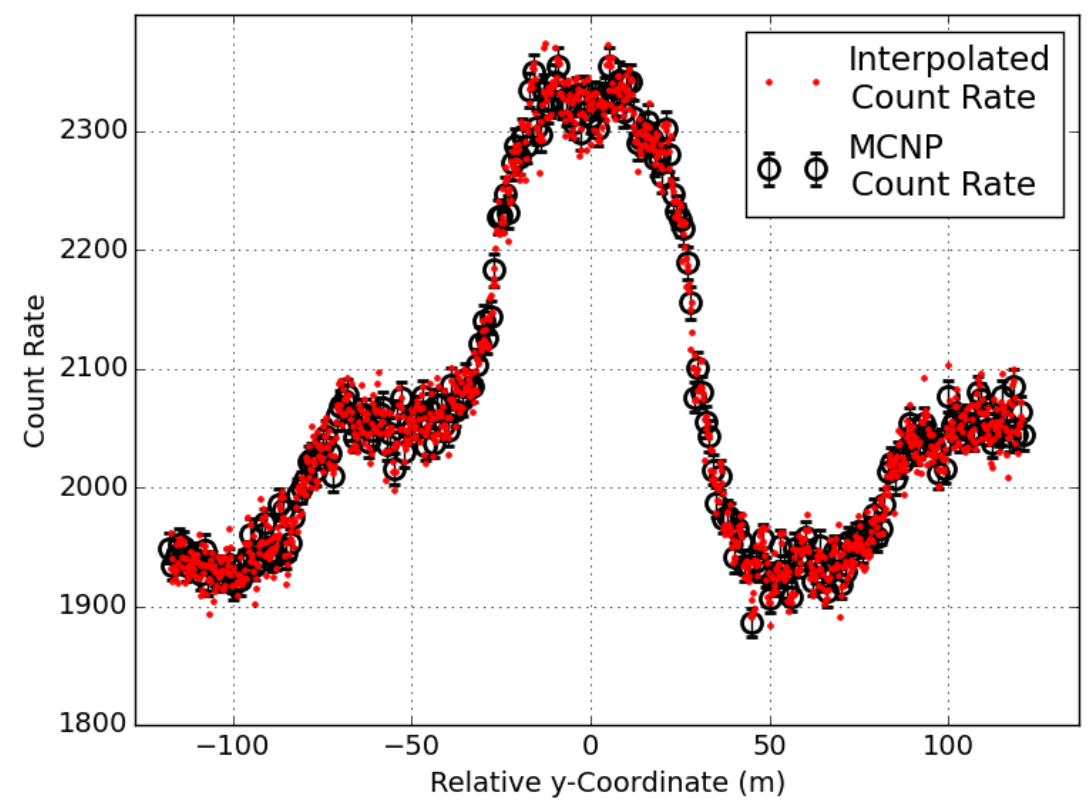

Fig. 13. MCNP and interpolated background count rate as a function of discrete calculation position. The black circles represent an individual MCNP calculation position. The red points are the result of using an assumed detector speed $(4 \mathrm{~m} / \mathrm{s})$ and integration time $(0.2 \mathrm{sec})$ to interpolate between the MCNP points.
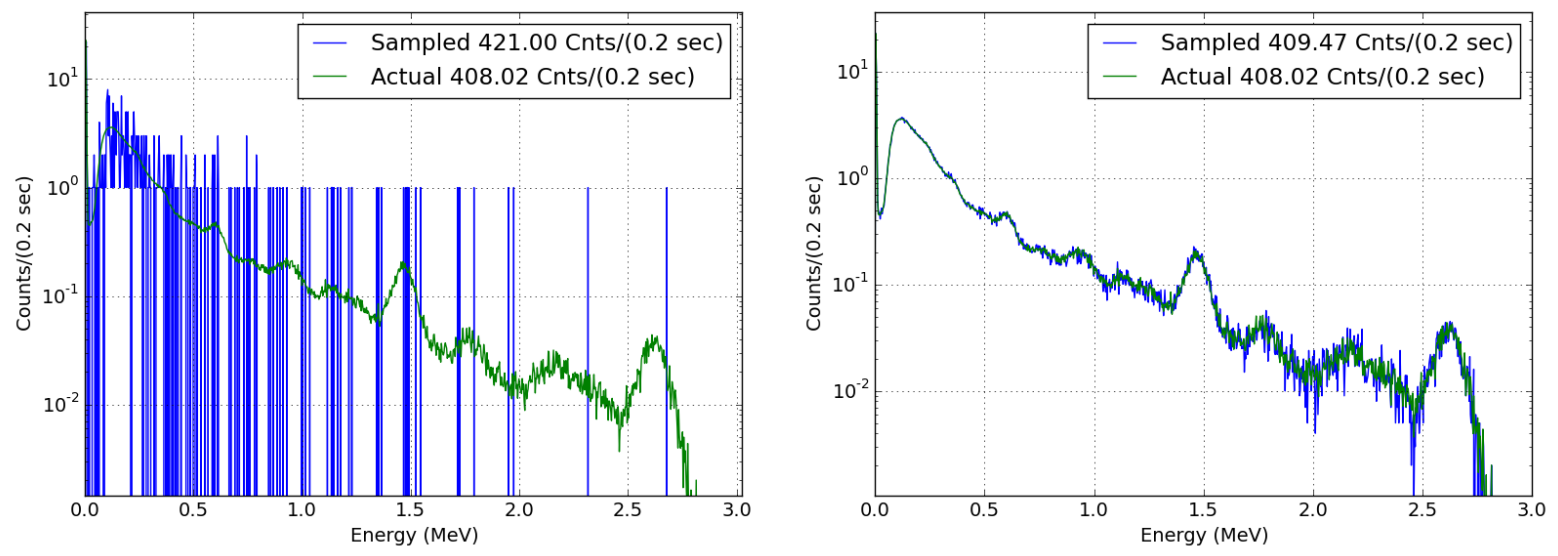

Fig. 14. MCNP and sampled background spectra. (Left) A one-second sample and (right) 1000 one-second samples averaged together. The green line represents the MCNP simulation spectrum and the blue the sampled spectrum. 


\subsection{CREATION OF MOVING TIME WINDOWS FOR SYNTHETIC DETECTOR OUT- PUT}

For a designated detector speed and integration time, each detection algorithm is run with a predefined foreground and background integration time (Fig. 15). In all detection algorithms, data in the foreground time window are compared with data from the background time window to make the determination of the presence of a source.

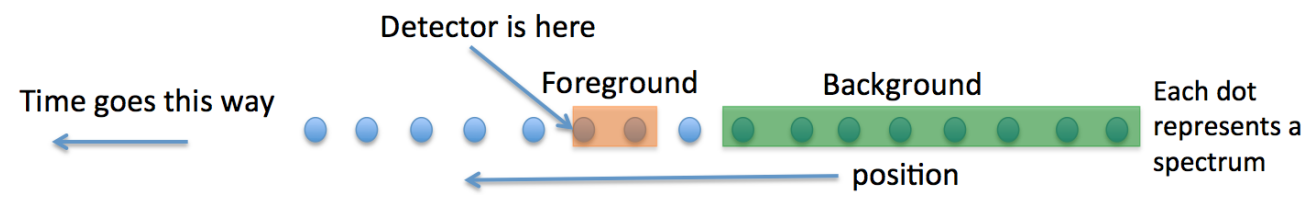

Fig. 15. A cartoon depiction of the data analysis of synthetic time series data. For time moving from right to left, each point represents a single position with an associated integration time and energy spectrum. Analysis integration windows of varying length for foreground (orange) and background (green) can be defined.

If the foreground and background time windows are too small, the probability of a false alarm is high, since two data sets containing poor statistics are being compared. If the time windows are too large, the system becomes less sensitive to quick changes in the count rate, which may be due to a radioactive source. Separate time windows can be created using different energy spectrum regions. Two energy windows are used in this work, a total energy spectrum and a source photo peak. For ${ }^{137} \mathrm{Cs}$, the energy window is defined as 652-672 keV. For HEU, the photo peak window is defined as 150-220 keV. False alarm and probability of detection analyses are conducted for a number of foreground and background windows for both total count rates and the defined energy window count rates.

\section{ANALYSIS OF ALGORITHM PERFORMANCE ON SYNTHETIC DATA}

This section describes the basic analysis approach taken to evaluate detector performance based on synthetic data as described in Sec. 3.4. The basic steps performed in the analysis process include the following.

1. Synthetic data time series are generated (discussed in Sec. 3.4).

2. Time windows are constructed, consisting of foreground and background data (discussed in Sec. 3.6).

3. As depicted by the cartoon in Fig. 15, at each time series data point, the algorithms are executed using a selected set of foreground and background time windows (discussed in Sec. 4.1). The execution of the algorithm results in either an alarm or no alarm status. These results are tallied and summarized in Sec. 4.3.

4. The analysis moves to the next time series data point. Foreground and background windows are updated, and algorithms are run again. This process is repeated for all data points created on the street.

This section first discusses the evaluated algorithms, and then it discusses the considered parameter space (such as speed, threat source strength, etc.). Finally, the algorithms performances for a collection of parameters are presented. 


\subsection{ALGORITHM DEFINITIONS}

In order to study the probability of alarms, as well as false alarm rates for the scenarios operationally modeled for this project, some form of a gamma detection algorithm must be used. However, due to the proprietary nature of many of the widely known and/or used detection algorithms, detections for the operational models in this report are performed using the widely known and implemented $k$-sigma algorithm. A second algorithm, referred to as WAVRAD [11], was made available to this project by the Remote Sensing Laboratory. The same operational models are examined by both algorithms and results are presented and compared. Both the $k$-sigma and WAVRAD are briefly described in the following sections. The results for each of these algorithms are discussed in Sec. 4.3.

\subsection{1 $k$-sigma algorithm}

The $k$-sigma algorithm can be performed using gross counts (entire energy spectrum) or a spectral region of interest (energy window). A mean background count rate (BG) is calculated by summing each time series energy spectrum or energy window count rate (CR) in the background window defined between time samples $i$ and $i+N$, where $N$ is the length of time of the background window:

$$
B G=\frac{1}{N} \sum_{j=i+1}^{i+N} C R(j) .
$$

From the distribution of these Poisson background count rates, deviations can be calculated using a standard Gaussian standard deviation, usually denoted by $\sigma$. Foreground energy spectrum count rates are then calculated using the foreground window (FG) using a selection of the most recent time samples $i$, where $M$ is the length of the foreground window:

$$
F G=\frac{1}{M} \sum_{j=i+1}^{i+M} C R(j) .
$$

The deviation of the FG window from the BG window in terms of the number of sigma values is then

$$
k=\frac{F G-B G}{\sigma} .
$$

Figure 16 illustrates a use case for the algorithm implementation. The red dots represent the count rate at each time interval, while the red line indicates the foreground window mean value. The threshold values for alarms determined by a selected $k$-sigma value for the background window are indicated by the blue region. An alarm occurs when the foreground mean (red line) exceeds the alarm threshold (blue region), as is shown around the position of zero on the horizontal axis.

\subsubsection{WAVRAD}

A short description of the WAVRAD algorithm is now presented based on [11]. WAVRAD is a shape-based detection algorithm that uses "recent" background measurements to determine the expected shape of the gamma-ray energy spectrum (the background window in Fig. 15). The algorithm then looks for deviations of the expected shape in a foreground window.

There are a few motivations toward using a shape algorithm. Capturing the effect of multiple peaks as well as changes in the continuum due to partial scatters in the detector requires a range of energy channels. 


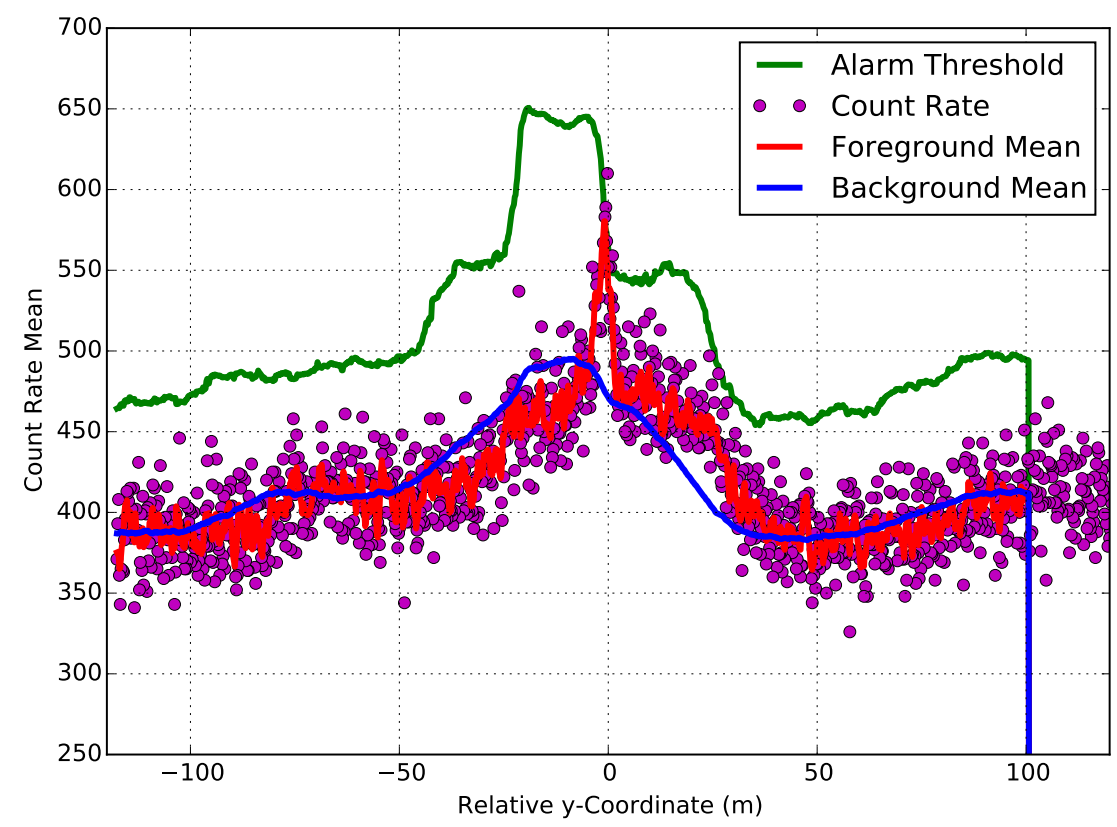

Fig. 16. An example plot of an interpolated count rate, foreground and background mean values, and alarm threshold for a specific detector speed and threat source strength. Since the detector moves from positive to negative relative y-coordinate (right to left), the alarm thresholds are not defined until enough points have entered into the background window (at about a relative y-coordinate of $100 \mathrm{~m}$ ).

At the same time and especially for time series data, the individual statistics of an energy bin will typically be very low.

Using the fact that a gamma energy spectrum is a collection of independent Poisson random variables, a linear transformation is applied to the spectrum using different Gaussian averages based on an estimated resolution. In effect this smooths the spectrum and concentrates effects spread out over multiple channels into a single channel. Since this is just a linear transform of Poisson random variables, the statistics of the transformed spectrum is readily understood, though the inclusion of multiple channels in the transform does introduce covariant errors that must be accounted for in the analysis. This transform is invertible, so none of the primary information is lost.

Typically, WAVRAD assumes a detector response using the standard Gaussian resolution $\sigma(E)$ :

$$
\sigma(E)=a_{0}+a_{1} \sqrt{\left(E+a_{2} \cdot E^{2}\right)},
$$

where $E$ is the energy/channel, and $a_{0}, a_{1}$, and $a_{2}$ are fit parameters. For data arranged in a time series (such as the synthetic time series used for this analysis), a background shape is determined by summing a number of seconds of background data. WAVRAD typically uses 30 or 60 seconds. The foreground is typically taken to be the most recent few seconds, though the exact choice is application dependent. Using the background as the expectation and the foreground as the measurement, mathematical deviations in the transforms are calculated. The "deviation from expectation" values can be plotted for each bin of the energy spectrum. These deviations are then analyzed in order to determine if identified deviations from expectation correspond to actual gamma spectrum anomalies (i.e., sources). These concepts are applied to 
the analysis presented in Sec. 4.3.2. For the purpose of this report, WAVRAD is treated as a black box, meaning that data were provided to the algorithm and the results were taken as is. The identical synthetic data sets are used for both $k$-sigma and WAVRAD algorithms to permit a more straightforward comparison of detection results.

\subsection{DISCUSSION OF THE ALGORITHM PARAMETER SPACE}

There is a large, multidimensional set of parameters explored in this algorithm analysis. All algorithms are run over two distinct synthetic data sets: those containing parked cars and those without parked cars. For each of these sets, the following parameters are explored: speed, threat source strength, foreground integration time, background integration time, and detection threshold. For ${ }^{137} \mathrm{Cs}$, the values of source strength explored range from 0 to $100 \mu \mathrm{Ci}$ in $10 \mu \mathrm{Ci}$ increments. For $\mathrm{HEU}$, a single spherical mass of $25 \mathrm{~kg}$ is considered.

In order to analyze the various algorithms' performance for probability of detection $\left(P_{D}\right)$ and false alarm rates (FAR), a set of 1000 synthetic interpolated count rates are uniquely sampled from the same source information. This is done for each combination of detector speed and source strength to be studied (listed in Table 3). Detection and false alarm probabilities can be determined for each speed and source combination by tallying the number of detections and false alarms per these 1000 test runs. In practice, the false alarm thresholds for this analysis are chosen such that there are zero false alarms per the 1000 sampled data sets (effectively a FAR $<0.001$ ).

The dimensions of the model's street represent at most 240 seconds of elapsed time when moving 1 $\mathrm{m} / \mathrm{s}$. In order to have viable background windows within the 240 meters of the modeled city street, the speed of the detector must be considered. Table 4 lists the amount of time available for a given speed. Since the source is placed at nearly the center of the city street, half of the total transit time up of the street is available for the background window.

Table 3. Values for the variables used in synthetic data set generation for algorithm evaluation.

\begin{tabular}{|c|c|}
\hline \hline Variable & Values \\
\hline Speed $(\mathrm{m} / \mathrm{s})$ & $1,2,4,5,6,7,8$ \\
${ }^{137} \mathrm{Cs}$ Source Strength $(\mu \mathrm{Ci})$ & $10,20,30,40,50,60,70,80,90,100$ \\
\hline \hline
\end{tabular}

In general, for a given speed the combination of FG and BG windows are restricted such that their sum does not exceed the times listed in Table 4 . The maximum speed considered is $8 \mathrm{~m} / \mathrm{s}$, in which the detectors passes from one end of the street to the other in about 30 seconds.

For $k$-sigma, algorithm performance is calculated for the foreground and background windows outlined in Table 5. In addition, each of these foreground/background combination sets is varied for a number of speeds (Table 3). For the case of the ${ }^{137} \mathrm{Cs}$ source, the source strengths are also varied so that a probability of detection can be expressed in terms of both source strength and speed. For each of the possible combinations of foreground window, background window, source strength, and detector speed a single optimal threshold $(k)$ is determined. In addition (and quite importantly), two large simulation data sets are created with parked cars and without parked cars. The entire analysis is conducted over both with and without parked car data sets in order to analyze the effect of street clutter on detection performance. The importance of street clutter (such as parked cars) on detection is well known operationally and is well illustrated in Fig. 9. 
Table 4. Dwell time for the modeled city street for different speeds. Since the source is placed at essentially the center of the street, the time available for background detection windows is half of the total distance transit time.

\begin{tabular}{|c|c|}
\hline \hline Speed (m/s) & time to source $(\mathrm{sec})$ \\
\hline 1 & 120 \\
\hline 2 & 60 \\
\hline 3 & 40 \\
\hline 4 & 30 \\
\hline 5 & 24 \\
\hline 6 & 20 \\
\hline 7 & 17.1 \\
\hline 8 & 15 \\
\hline 16 & 7.5 \\
\hline \hline
\end{tabular}

Table 5. Foreground and background windows considered in the analysis. Combinations of foreground and background times are restricted due to a detector speed and the finite dimensions of the modeled city street.

\begin{tabular}{|c|c|}
\hline \hline Foreground (sec) & Background (sec) \\
\hline 1 & $5,10,20,30,45,60$ \\
\hline 2 & $10,20,30,45,60$ \\
\hline 3 & $10,20,30,45,60$ \\
\hline 5 & $10,20,30,45,60$ \\
\hline \hline
\end{tabular}

For the HEU case, only a spherical mass of $25 \mathrm{~kg}$ is considered for a threat source. The parameters of foreground window, background window, and detector speed are all calculated in order to arrive at an optimal threshold $k$ value.

As previously presented, Fig. 16 shows an example of a single data set corresponding to a single speed and threat source strength. In this figure the count rates are shown along with the averages of the foreground window (red line) and background window mean and standard deviation spread of the averaged background points (blue band). Alarms (either correct or false) are defined to occur when the foreground mean value exceeds the alarm threshold defined by the blue band. This occurs in Fig. 16 for a true alarm on ${ }^{137}$ Cs peak source near the relative $y$-coordinate value of zero.

\subsection{ALGORITHM PERFORMANCE}

The detection algorithms are tested against synthetic data sets constructed in the manner previously described. The probability of alarm is plotted for different detector speeds and source strengths for a given set of algorithm parameters. The parameters varied in this study include the foreground and background window lengths, as well as the alarm thresholds which are defined in different ways for each of the two algorithms evaluated. The details of these thresholds are discussed in the relevant algorithms result subsection. 


\subsection{1 $k$-sigma results}

For the $k$-sigma algorithm, a full set of $k$ values (defined in Equation 4) are examined. Optimal $k$-sigma values vary depending on the operational parameters being considered. For a given set of parameters: FG time, BG time, speed, and source strength, an optimal $k$-sigma value can be determined. Figure 17 illustrates how an optimal $k$-sigma value is chosen for a fixed set of parameters. In Fig. 17, the probability of false alarms (green curve) is determined by running the chosen parameter set over synthetic data without a source. The probability of alarm (blue curve) is then calculated with the same parameter set using synthetic data with combined source combined with background. At $k=2$ there is a large probability of false alarm which dramatically drops at $k=3$, where the FAR is near zero but the detection rate is still near $100 \%$. The optimal value is highly dependent on source strength and FG and BG window choices. FG and BG choices are also strongly affected by the speed of the detector pass-by. For the case considered in the figure, the optimal $k$ values is somewhere between $k=2$ and $k=3$ and is determined by examining $k$ values in smaller increment steps.

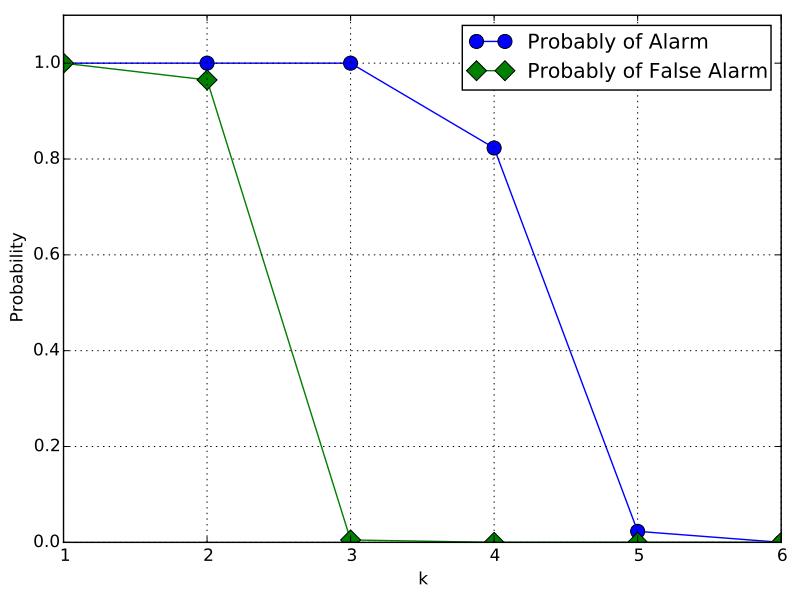

Fig. 17. An example plot from the synthetic data analysis for probability of alarm and probability of false alarm vs. a $k$-sigma value for a specific choice of FG, BG, speed, and source strength. The optimal value is found for a maximum probability of alarm for the defined tolerable level of false alarms.

The impact of the choice of a $k$-sigma value on false alarms and probabilities of detection versus source strength are shown in Fig. 18. This plot is for the detection of ${ }^{137} \mathrm{Cs}$ using a spectral energy window of $620-720 \mathrm{keV}$, and using a 1 second FG window and 20 second BG window. Speeds from 1 to $8 \mathrm{~m} / \mathrm{s}$ in 1 $\mathrm{m} / \mathrm{s}$ increments are plotted, though as shown in Table 4, the faster speeds are not applicable for this choice of FG and BG time windows. In the left plot, the false alarm rates, denoted by dashed lines, are at the level of $10 \%$ or less. Changing the alarm threshold from $k=2.5$ to $k=2.75$ eliminates the false alarms to a level below 0.001. Elimination of the false alarms from the detection curve pushes the probability to detect to larger value source strengths as expected.

A comparison of spectral window and full spectrum probability-to-detect curves is shown in Fig. 19. The spectral window choice of $k=2.5$ (left plot) and the full spectrum choice of $k=4.0$ (right plot) were separately determined by choosing the lowest $k$-sigma value that reduced false alarms to the predetermined level (FAR < 0.001). As expected the use of a spectral window for detection performs significantly better than the full spectrum. Table 6 lists the $50 \%$ probability of detection values for the $k$-sigma algorithm. For 

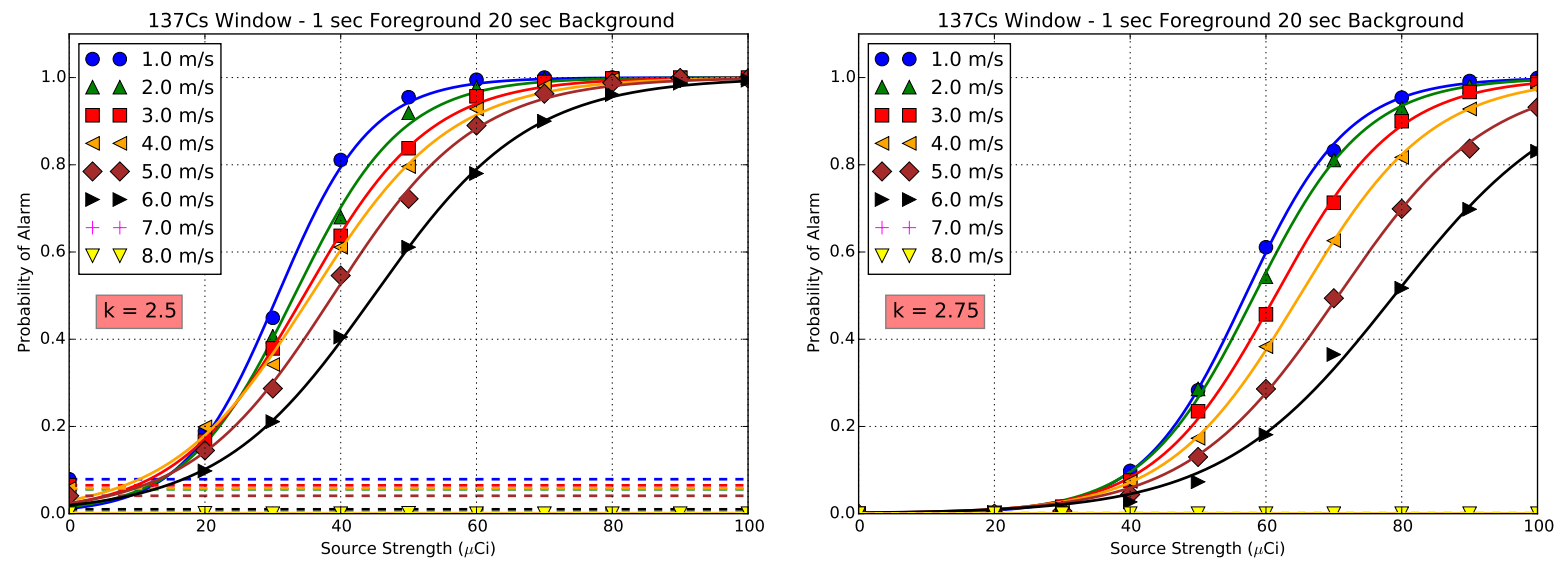

Fig. 18. Probability of alarm distributions (with cars) for two different $k$-sigma choices for detection scenarios with a 1 second FG and 20 second BG. False alarm rates are denoted by horizontal dashed lines. An increase from $k=2.5$ (left plot) to $k=2.75$ (right plot) reduces the FAR to the predefined level. At the same time the probability of alarm shifts to the right, indicating reduced sensitivity to source detection.

the $25 \mathrm{~kg}$ spherical HEU source no table is provided since detection was $100 \%$ for all parameter sets considered, however the background levels were varied for this source in order to track the change in the probability of detection curves as a function of background level.

Table 6. ${ }^{137} \mathrm{Cs}$ source strengths (in $\mu \mathrm{Ci}$ ) required to reach a $50 \%$ detection probability for the $k$-sigma algorithm. Data is determined from fits in Fig. 19. In the fits, $k=2.5$ was used for the ${ }^{137} \mathrm{Cs}$ spectral portion, while $k=4.0$ was used for the full spectrum. For $\mathrm{FG}=1$ second and $\mathrm{BG}=20$ seconds, speeds greater than 4 $\mathrm{m} / \mathrm{s}$ are excluded due to limited dwell time.

\begin{tabular}{ccc}
\hline \hline Speed & $\begin{array}{c}\text { Spectral Window } \\
620-720 \mathrm{keV}\end{array}$ & Full spectrum \\
$(\mathrm{m} / \mathrm{s})$ & $\mu \mathrm{Ci}$ & $\mu \mathrm{Ci}$ \\
\hline 1.0 & 38.4 & 90.7 \\
\hline 2.0 & 42.4 & $>100.0$ \\
\hline 3.0 & 48.3 & $>100.0$ \\
\hline 4.0 & 55.2 & $>100.0$ \\
\hline 5.0 & $\mathrm{NA}$ & $\mathrm{NA}$ \\
\hline
\end{tabular}

Total background levels were varied from $100 \%$ to $300 \%$ of nominal value. Figure 20 shows the probability for detection curves as a function of speed for the different background levels. The curves are for a choice of $\mathrm{FG}=1$ second and $\mathrm{BG}=20$ seconds windows and with a $k$-sigma $=3.4$ corresponding to a FAR $<0.001$. As expected the detection sensitivity is strongly affected by the level of background, with a very strong reduction in probability of alarm with increased speed. For instance, comparing probabilities of alarm for a default background case and a $200 \%$ default background level shows that at $6 \mathrm{~m} / \mathrm{s}(13.4 \mathrm{mph})$ the probability to alarm is reduced from nearly $100 \%$ to less than $40 \%$. Given the large variability in background levels observed in real-world detector data considered in conjunction with the difficulty in 

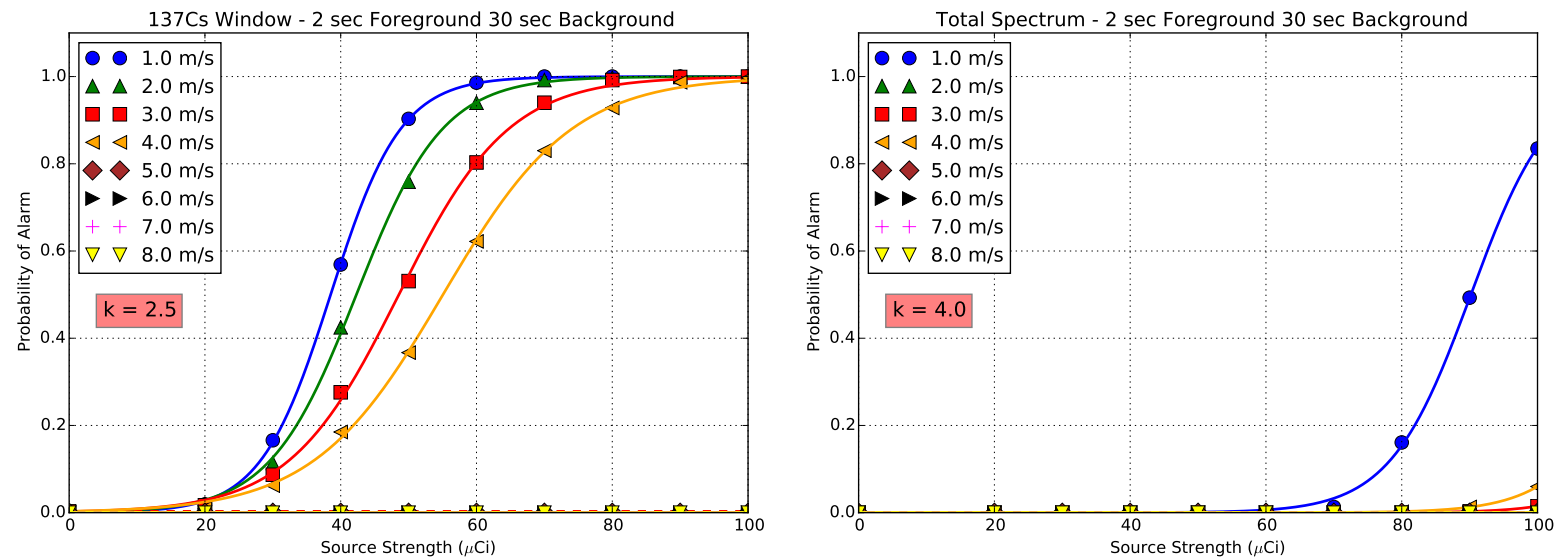

Fig. 19. Probability of alarm distributions for the $k$-sigma algorithm (with cars) for a ${ }^{137} \mathrm{Cs}$ spectral window 620-720 keV (left plot) and full spectrum (right plot).

accurately modeling backgrounds to match a specific geographical location to within a factor of two, the results of Fig. 20 suggest that use of near-real-time operational modeling to provide accurate in situ awareness is unlikely.

\subsubsection{WAVRAD results}

For the purposes of this paper, the WAVRAD algorithm was run essentially as a black box. Data were provided to the algorithm and results were output. The WAVRAD algorithm considers many FG and BG windows in its analysis. A final figure-of-merit (FOM) is provided that serves as a detection threshold, similar to that of a $k$ value for the $k$-sigma algorithm. This FOM can be adjusted up and down to set the false alarm rate to a desired level to examine probability of detection values.

Common FG and BG windows were chosen in order to compare detection probabilities between the WAVRAD and $k$-sigma algorithms. These results are listed in Tables 6 and 7 .

Figure 21 shows results for the WAVRAD algorithm run with a 2 second foreground window and a 30 second background window. The foreground and background windows were chosen based on the $k$-sigma performance for the ${ }^{137} \mathrm{Cs}$ spectral window. The probability of alarms is calculated by running the algorithm over the same 1000 sampled passes of the detector by the source.

The WAVRAD results for HEU detection in Fig. 22 show the expected impact of parked cars on detection probabilities. The solid blue line is for probability of detection without cars, while the dashed red line is for probability of detection with cars. The $25 \mathrm{~kg}$ HEU is detected at all speeds considered when there are no parked cars on the road. The addition of parked cars severely degrades detection probabilities, with decreasing performance with increased detector speeds. At the fastest speed considered, $8 \mathrm{~m} / \mathrm{s}$ (about $18 \mathrm{mph}$ ), the reduction in detection drops from $100 \%$ for the no car case, to less than $5 \%$ for the parked car case. 


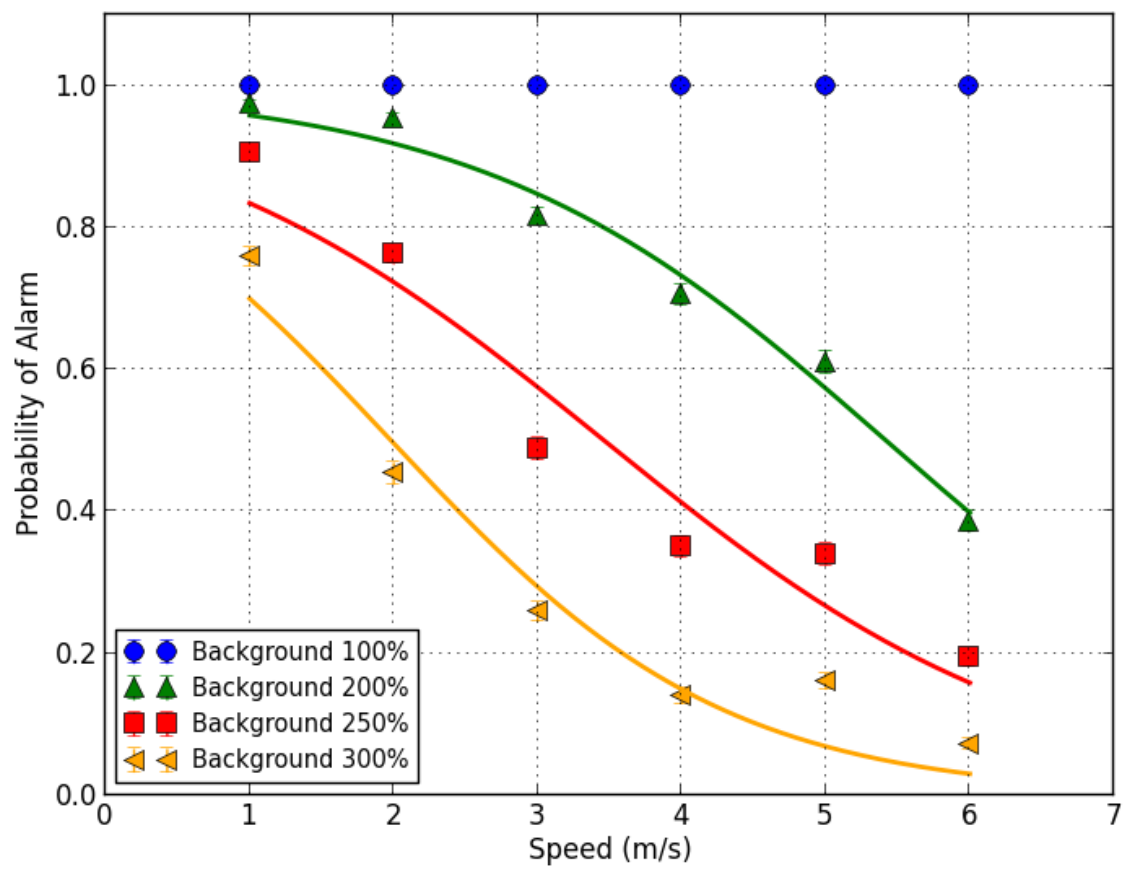

Fig. 20. Impact of background variation on probability of detection for $25 \mathrm{~kg}$ HEU (with parked cars). 

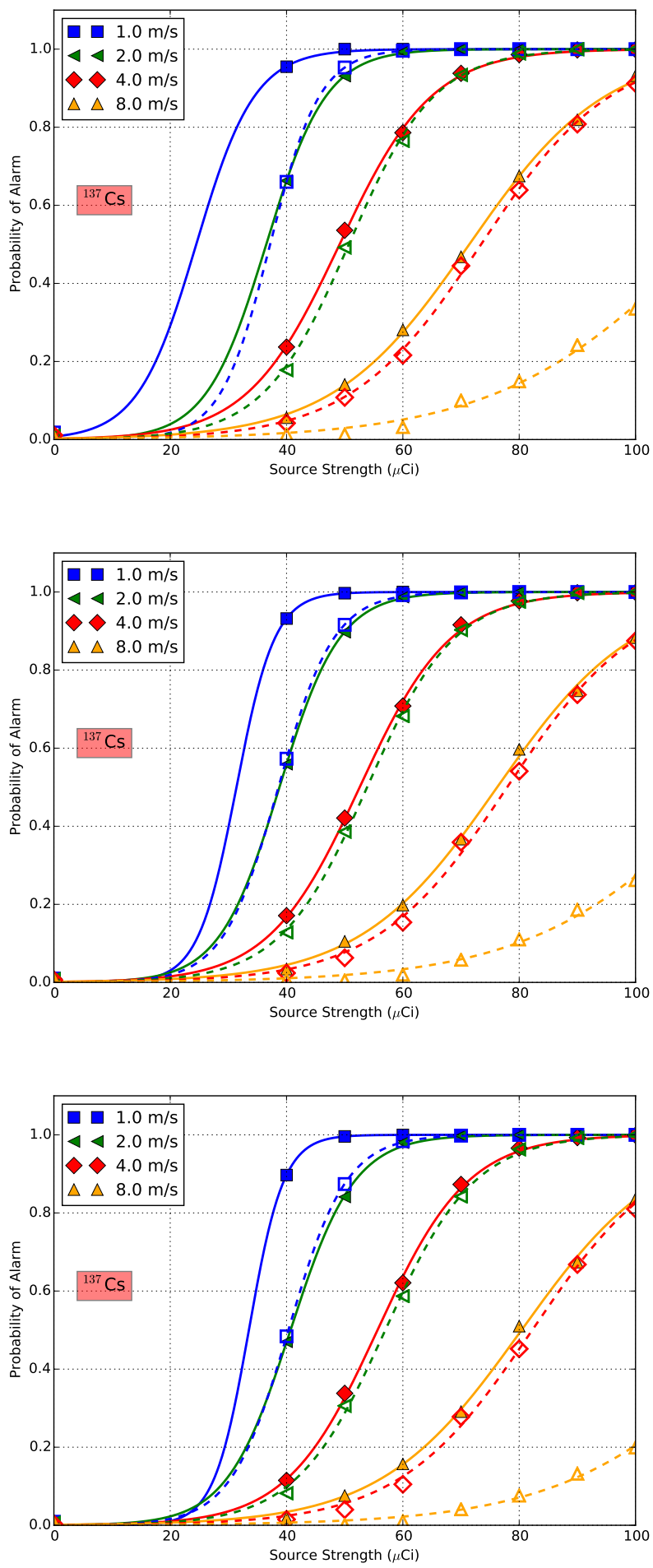

Fig. 21. WAVRAD results for ${ }^{137}$ Cs for FOM's of 1.1 (top), 1.15 (middle), and 1.2 (bottom). The probability of detection values listed in Table 7 use FOM=1.15. Solid lines are for detection without cars, while dashed lines are for calculations with cars. 


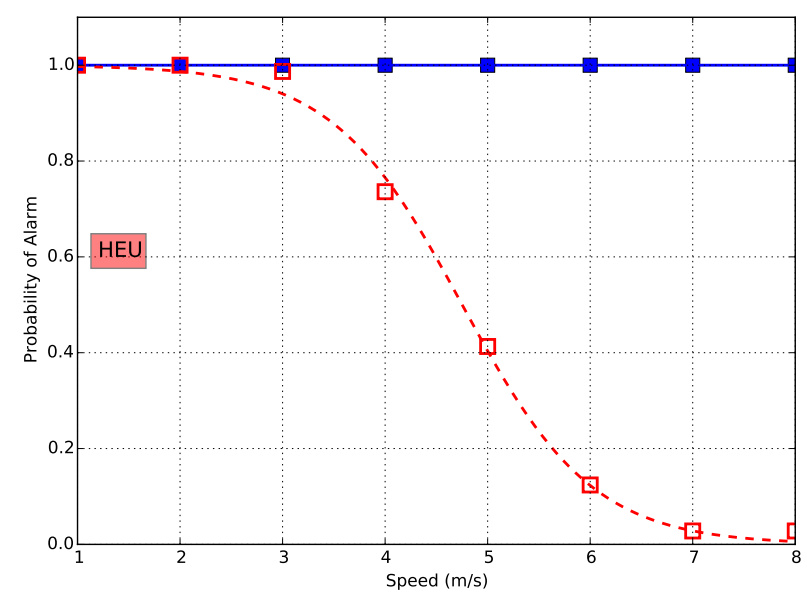

Fig. 22. Probability of alarm for HEU as a function of speed. The blue line is for without parked cars; the dashed line is for parked cars. The reduction in the detection of the HEU for increasing speeds dramatically illustrates the increased shielding effect of parked cars.

Table 7. ${ }^{137}$ Cs source strengths required to reach a 50\% detection probability using the WAVRAD algorithm, FOM=1.15.

\begin{tabular}{ccc}
\hline \hline & \multicolumn{2}{c}{ Source Strength at $\mathrm{P}_{D}=50 \%$} \\
Speed $(\mathrm{m} / \mathrm{s})$ & No Cars & With Cars \\
\hline 1.0 & 31.4 & 38.6 \\
2.0 & 38.7 & 53.9 \\
4.0 & 52.6 & 78.0 \\
8.0 & 76.2 & $>100.0$ \\
\hline \hline
\end{tabular}

\subsection{EXPECTED PERFORMANCE OF ALGORITHMS ON REAL DATA}

It is understood that, despite the relative care taken to model background radiation levels for a city street, the simulation data drastically underestimates the fluctuations found in real-world background data collects. Part of the motivation to include Fig. 1 was to illustrate the well known large fluctuations in the simple background count rate due to various man-made and natural structures. To illustrate the discrepancy in the variation of the modeled city street count rates versus that of a real-world city street data set, the algorithms used in the previous sections were run on real-world background data.

Eight hours of data taken from an RSL mobile measurement [12] are shown in Fig. 23. For the same $k$-sigma value used in Table $6(k=4.0)$, the RSL data registered 197 false alarms. This averages out to 0.00684 alarms $/ \mathrm{sec}$. Given that the modeled city street represents at most 240 seconds (at $1 \mathrm{~m} / \mathrm{s}$ ), this would suggest that the alarm rate is 0.00684 alarm $/ \mathrm{sec} \cdot 240 \mathrm{sec}=1.64$ alarms per 240 meters of roadway at $1 \mathrm{~m} / \mathrm{s}$. 


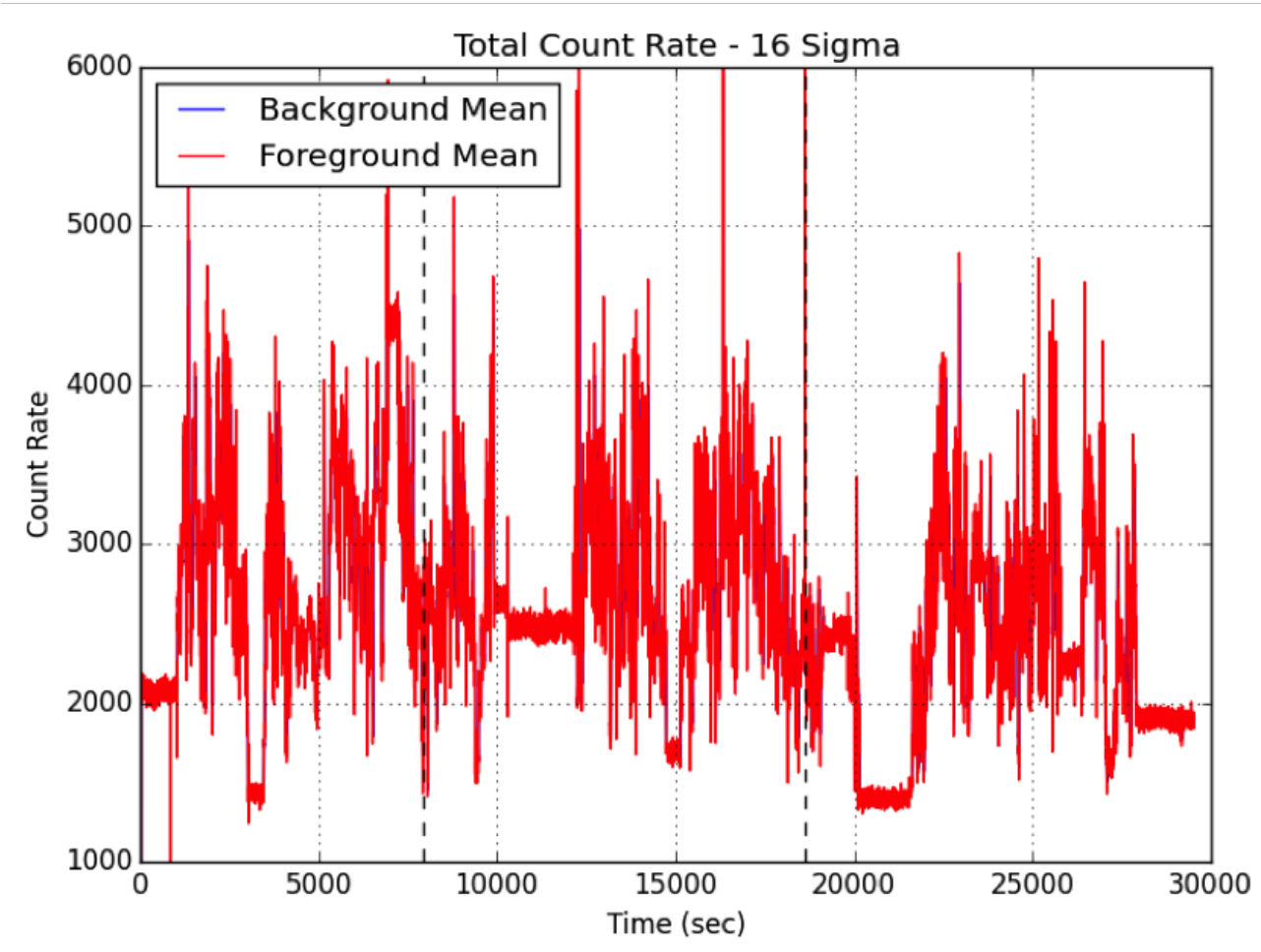

Fig. 23. Count rates from RSL mobile data [12] based on the sum of four 2 " $\times 4$ " $\times 16$ " NaI detectors. The dashed lines are for $k$-sigma alarms based on a $16-\sigma$ foreground deviation from the background window. Additional details are provided in the text. 


\section{CONCLUSION}

This report concludes by summarizing key findings and describing recommended software next steps.

\subsection{KEY FINDINGS}

1. The primary contributor to the background rates is the road surface, followed by surrounding buildings and curbs/sidewalks. For the detection scenarios considered, the impact of cosmic rays on operational count rates is insignificant.

2. Dynamic background components, represented in this work by parked cars, have a significant impact on detection.

3. Model size matters, especially when transporting diffuse background sources. For a large city block of a few hundred meters in length, significant computational resources were required. Still, the total amount of operational search time represented by this simulation effort is just a few minutes.

4. The modeling of backgrounds to exhibit realistic features requires an unrealistically immense amount of detail in the physical geometry. However, it is the very nature of the details in the real environment that impacts detection (and thereby false alarm and probability of detection rates).

5. Background levels have significant impact on the probability of detection, as illustrated in Fig. 20. Modeling these background levels to match a particular situation is an extremely difficult proposition and requires significant computational resources and/or time.

6. Detection depends on the algorithm and the details of the algorithm implementation (e.g., threshold, speeds, source size, energy bins, time bins).

7. Different sources are detected with different sensitivities. For instance, when using $k$-sigma, different optimal detection thresholds exist for different sources, complicating the analysis in operational search.

8. If, through an exhaustive effort and against all odds, one could possibly construct a sufficiently detailed model, the calculation time required does not yet permit near-real-time results, even using advanced discrete ordinate codes for the radiation transport.

\subsection{SOFTWARE DEVELOPMENT STRATEGY}

Modeling and simulation of background for radiation detection problems has been used for a long time in many areas, including system design, parametric studies, and exercise data creation. With ever faster computers, some have thought of using detailed modeling to predict and subtract background from detector count rate measurements in real time. Unfortunately, the discussion in the previous section has highlighted the fact that so-called "static" background sources can have a large variability in space and time. Even ignoring the computation time, the data needed as input for a first-principles radiation transport simulation-geometry, materials, and source distribution as a function of space and energy — could not be determined and fed to a transport code in a time remotely close to real time. For fixed detectors, the surrounding environment could be well characterized and modeled, but other factors such as the daily changes in pressure and humidity/rain or occlusion (static or random) from passing traffic make quick and accurate modeling difficult. 
Creating large databases of measured background for important areas can give some insight into the expected range of background levels for a particular detector but most likely could not be used to operationally model background well enough to correct new measurements on-the-fly. If everything that affects the background was exactly the same as when the database background was recorded, that record is still only good for the same detector with the same electronic settings that made the database measurements. A measurement of background includes the detector response, which varies among detector types and could vary slightly among several detectors of the same type. A background measurement does not describe the source distributions of the background radiation that contribute to the reading so previously measured background using one type of detector would not be very helpful in accurately predicting the background that a different type of detector would measure.

With the large range of variability of the background radiation over small distances and time periods, attempts to model and subtract background from measurements may cause more harm than good. What if the background is estimated to be higher than the real measurement? How would negative net counts impact the detection algorithms? Other strategies to reduce the impact of background-such as subtracting out spectral shapes of common background isotopes or looking for changes in normalized spectral shape are more straightforward and introduce far less uncertainty into the detection process.

Modeling and simulation still has a large role to play in characterizing detection systems-just not in real time. There are improvements that can be made but the emphasis should not be in codes and models that generate precise but inaccurate neutron and photon background source distributions for any spot on the globe. For example, improving the resolution of the kilometer-scale USGS average soil concentrations of $\mathrm{K}$, U, and Th would likely not contribute much to estimating the background at a specific location when the changes over a few meters can be a factor of two or three due to natural variations in geology or man-made structures. Recent developments in radiation transport codes such as MCNP [1] and SCALE/MAVRIC $[8,10]$ already provide flexible source modeling capabilities. However, having automated background sources within the codes may be helpful to users, but it must be recognized that the users will still need to increase/decrease the modeled background over the expected range of what the detection system would likely experience. So, rather than more and more ultra-precise background models, changes to the input of the programs that allow for easier entry of specific background components would be more useful.

Likewise, doing extensive mapping of background on every street in the country under different conditions and building an exhaustive database with the hopes of subtracting out a prior background count rate from a new measurement are likely not a worthwhile approach. It must be recalled that everything that moves with the detector (the measurement vehicle and perhaps the traffic in front and behind the vehicle) is part of the detector response function. If the new measurement is taken with a different detector changes or the measurement vehicle is different, the database background may no longer be relevant. Because of natural environmental changes (weather) and changes in the human-controlled environment (parked cars, traffic), the accuracy of the database background would always be suspect.

This report concludes with a few recommendations for future modeling actions and investment.

1. Standard sets of energy spectra and range of magnitudes should be created for each background contributor, including typical ranges of $\mathrm{K} / \mathrm{U} / \mathrm{Th}$.

2. For system model characterization, detection algorithms should be run with varying background levels and compositions

3. For the modeling a specific measurement and detection scenarios, background sources must be carefully characterized each time. 
4. Significant future improvement is expected from the ongoing work for improved algorithms. In addition to the inclusion of spectral information and spectral windows, spectral shape based methods should be explored. Methods to subtract out spectral shapes of K/U/T after fitting to peaks can be explored.

5. Given the inherent (and to some degree unsolvable) uncertainty in background levels for operational scenarios, exploration of more robust algorithms for detection is an area where more resources can be applied.

6. A combined backgrounds measurement and modeling campaign should be conducted with the goal of obtaining benchmark data against which model geometries and background levels can be precisely studied. As observed in this report, given the sensitivity of detection algorithm performance relative to background levels and the desire to use background modeling in operational scenarios, it is important to gain an understanding of the actual level of detail required in a model to be operationally useful. 


\section{References}

[1] X-5 Monte Carlo Team, "MCNP-A General Monte Carlo N-Particle Transport Code, Version 5. Volume I: Overview and Theory," LA-UR-03-1987, Los Alamos National Laboratory, Los Alamos, New Mexico (2003).

[2] J. C. Wagner and and A. Haghighat, "Automated Variance Reduction of Monte Carlo Shielding Calculations Using the Discrete Ordinates Adjoint Function," Nuclear Science and Engineering 128, 186 (1998).

[3] Haghighat and J. C. Wagner, "Monte Carlo Variance Reduction with Deterministic Importance Functions," Prog. in Nuc. Ener. 42(1), 25-53 (2003).

[4] J. C. Wagner, D. E. Peplow, and S. W. Mosher, "FW-CADIS Method for Global and Semi-Global Variance Reduction of Monte Carlo Radiation Transport Calculations," Nuclear Science and Engineering 176, No. 1, 37-57 (2014).

[5] S. W. Mosher, A. M. Bevill, S. R. Johnson, A. M. Ibrahim, C. R. Daily, T. M. Evans, J. C. Wagner, J. O. Johnson, R. E. Grove, "ADVANTG - An Automated Variance Reduction Parameter Generator," ORNL/TM-2013/416, Oak Ridge National Laboratory (2013).

[6] A. Nicholson, D. E. Hornback, B W. Patton, T. M. Miller, et. al., "Systematic Assessment of Neutron and Gamma-ray Backgrounds Relevant to Operational Modeling and Detection Technology Implementation," ORNL/LTR-2013/596 (2014).

[7] I. C. Gauld, G. Radulescu, G. Ilas, B. D. Murphy, M. L. Williams, and D. Wiarda, "Isotopic Depletion and Decay Methods and Analysis Capabilities in SCALE," Nuclear Technology 174(2), 169?195 (2011).

[8] SCALE: A Comprehensive Modeling and Simulation Suite for Nuclear Safety Analysis and Design, ORNL/TM-2005/39, Version 6.1, June 2011. Available from Radiation Safety Information Computational Center at Oak Ridge National Laboratory as CCC-785.

[9] H.H. Hakimabad, "Response Function of a $3 \times 3$ in. NaI Scintillation Detector in the range of 0.081 to 4.438 MeV”, Asian J. Exp. Sci., Vol. 21, No. 2, 2007, 233-237.

[10] D. E. Peplow, "Monte Carlo Shielding Analysis Capabilities with MAVRIC," Nuclear Technology 174(2), $289 ? 313$ (2011).

[11] private communication on WAVRAD, Thomas McCullough, Remote Sensing Laboratory, Andrews, November 2014.

[12] Private Communication, Matt Kiser, Remote Sensing Laboratory. 


\section{A COSMIC RAY CONTRIBUTIONS TO PHOTON BACKGROUND IN CITY BLOCK SIMULATIONS}

The focus of the city block simulations already described in this report is testing different detection algorithms to detect sources hidden along a street. The background radiation modeled is produced by the natural occurrence of potassium, uranium, and thorium (KUT) in man-made concrete streets and sidewalks, and concrete, brick, or granite buildings. Shielding is provided by the car the NaI detector is located in, cars parked along the side of the street, and the trashcan the sources are located in. In reality there is a second component to the background radiation, which is the background induced by galactic cosmic rays (GCR). This appendix details the effort to estimate the background due to GCR for these city block simulations.

The assumption at the outset of this analysis is the GCR induced photon background is small compared to the terrestrial photon background. This assumption is based on the amount of KUT in close proximity to the $\mathrm{NaI}$ detector in the model. If the scenario in this analysis was at sea or if a neutron detector was used instead of a photon detector, this assumption would not be true. At sea there is very little KUT in the surrounds, so the primary source of photons would be GCR. For neutrons, the GCR and Solar Particle Events (SPE) are nearly the only source of background.

The analysis of the background induced by cosmic rays was performed in two steps.

1. Start with a GCR source, consisting of protons and alphas $200 \mathrm{~km}$ above Earth and calculate the energy and angular dependent spectra of particles "entering the street" of the city block model.

2. Use the energy and angular dependent spectra calculated in the first step as sources to calculate the photon count rate in a $\mathrm{NaI}$ detector traveling in a car within the city block model.

The second step of this analysis must be repeated for each particle type deemed important to the NaI response.

\section{A.1 THE MODEL, GEOMETRY AND MATERIALS}

The city block geometry and materials described in Section 2.1 are the starting points for this analysis. For the first step of this analysis, materials had to be added to model additional soil below the street and sidewalk and to model the atmosphere above the tops of the building faces. An additional 5 meters of dry U.S. average soil [1] was included in the model to account for high energy cosmic rays and their progeny interacting with Earth and creating secondary radiation that is scattered back into the street. Since the first step of this analysis starts with GCR protons and alphas in low Earth orbit, altitude of $200 \mathrm{~km}$, the atmosphere was modeled in $1 \mathrm{~km}$ thick layers with different densities and compositions. This was necessary in order to simulate the shower of particles produced by GCR entering Earth's atmosphere. The original city block model included dry air, so a model from the Naval Research Laboratory [2] was used to produce dry air densities and compositions for each of these layers of the atmosphere. The layer nearest Earth was actually broken into 4 layers, $0.25 \mathrm{~km}$ each, to provide more fidelity near Earth's surface.

\section{A.2 THE GCR SOURCE}

NASA's 1-D transport code HZETRN-2010 [3] was used to generate the source spectra of protons and alphas $200 \mathrm{~km}$ above Earth. The parameters used to define the atmosphere and GCR are given in

Table A.1. The most important of these parameters, as far as the GCR source is concerned, are the location 


\begin{tabular}{|c|c|}
\hline Parameter & Value \\
\hline Location & New York City \\
\hline Temperature & $56^{0} \mathrm{~F}$ \\
\hline Air Pressure & $0.9936 \mathrm{~atm}$ \\
\hline Elevation & Sea Level \\
\hline Humidity & $0 \%$ Relative Humidity \\
\hline Solar Cycle & Solar Minimum (Oct. 1996) \\
\hline
\end{tabular}

Table A.1. Parameters of Base Computational Model

on Earth and date within the solar cycle. Remember that solar minimum, i.e. the minimum of solar activity like sun spots and coronal mass ejections, correlates to a maximum in GCR flux.

The actual proton and alpha spectra are plotted in Figure A.1. The total number of protons and alphas present in these spectra are $4.176 \times 10^{18}$ and $5.382 \times 10^{17}$ ( $88.58 \%$ and $11.42 \%$ of the total), respectively.

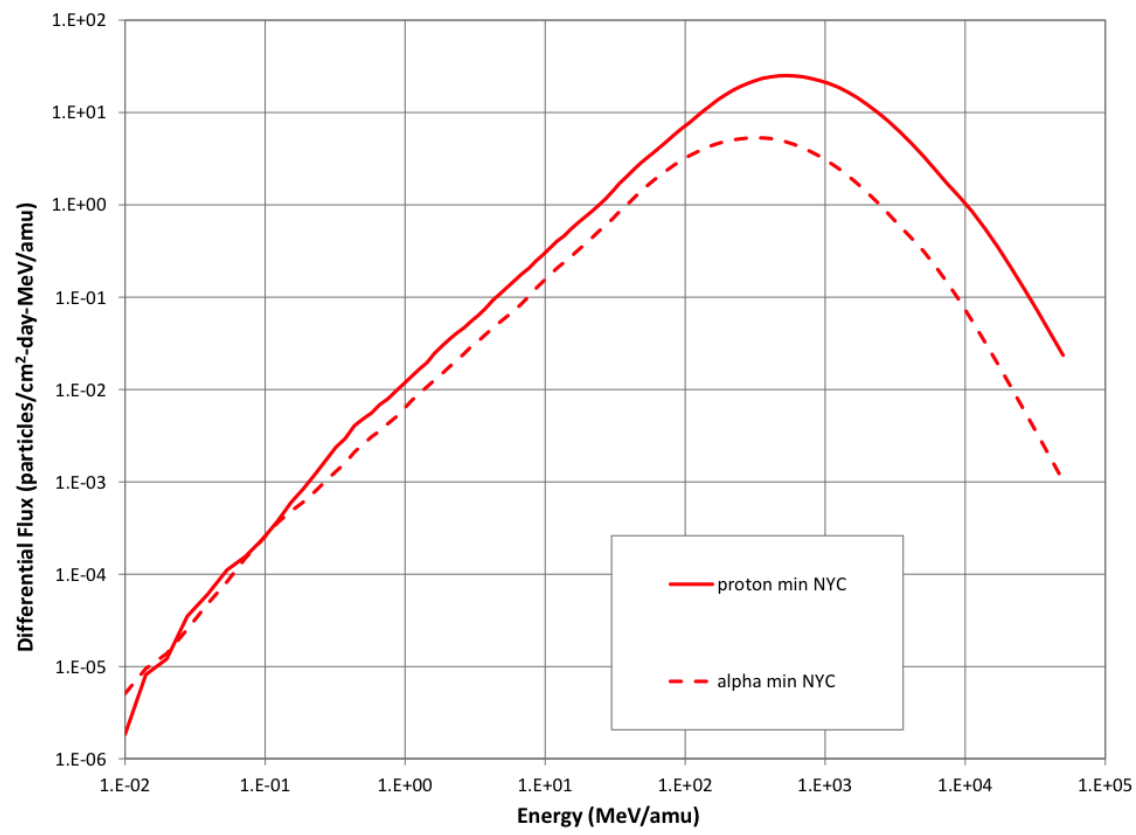

Fig. A.1. GCR proton and alpha spectra during the October 1996 solar minimum $200 \mathrm{~km}$ above New York City [3]

\section{A.3 STEP 1 RESULTS AND SOURCES FOR STEP 2}

The purpose of step 1 in this analysis is to calculate the energy and angular dependent spectra of particles "entering the street" of the city block model. A 3D view of the model without the additional ground or atmosphere layers is shown in Figure A.2. The view in Figure A.2 is looking down the street of the city block model with cars (in red) parked along the sides of the street. Before the step 1 simulations were started the geometry was modified to remove all the cars, those parked and the one with the NaI detector. Also shown in Figure A.2 is a rectangular parallelepiped drawn with black dotted lines that 
outlines the surfaces used for tallies in step 1 and therefore sources in step 2 of this analysis. Since the actual model used in step 1 of this analysis did not include any cars, the step 1 tallies and step 2 sources are symmetric on the $\mathrm{x}$-boundaries (parallel to the street, right and left) and y-boundaries (perpendicular to the street, front and back).

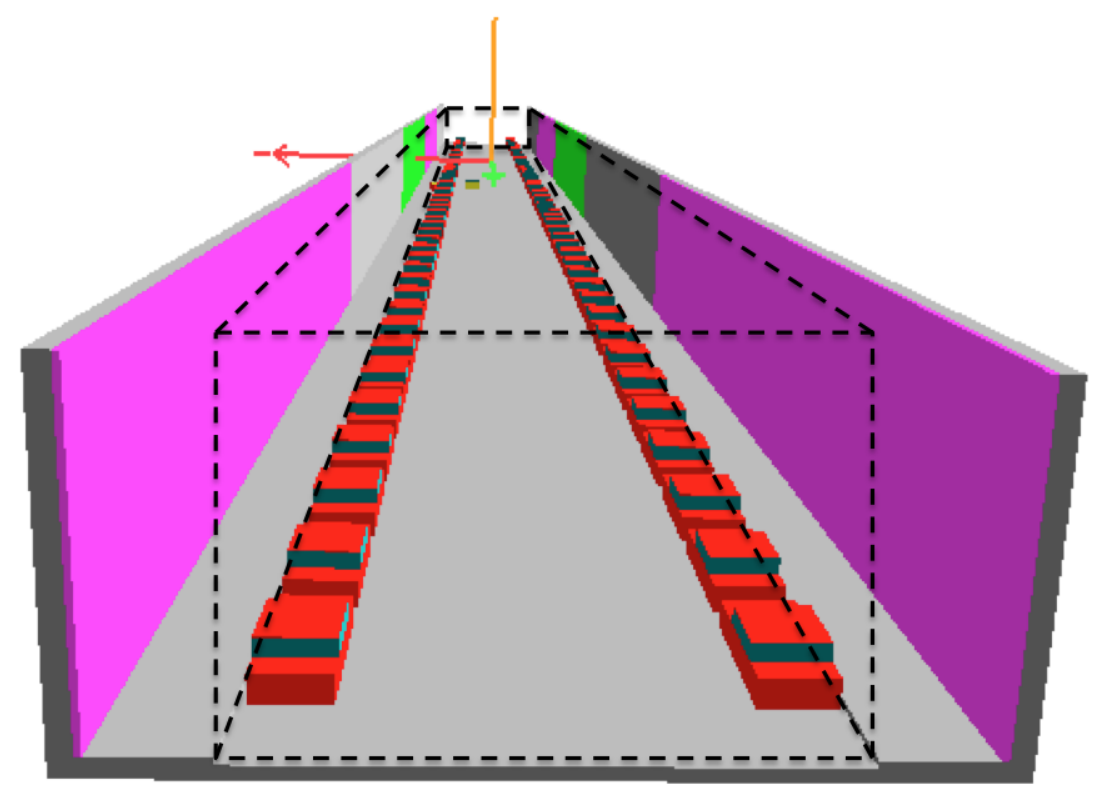

Fig. A.2. City block model with parked cars and black dotted lines outlining step 1 tally surfaces and step 2 source surfaces.

Figures A.3 through A.8 plot the spectra of neutrons, photons, electrons, protons, muons, and anti-muons that were tallied on these surfaces in step 1. The curves in these plots represent the total energy spectra on each surface. In other words, the computational results have been integrated over polar angles pointing into the rectangular parallelepiped. Five polar angular bins were used for the step 1 tallies, and the boundaries of the polar angular bins are 0 (normal to the tally surface), 18, 36, 54, 72, and 90 (parallel to the tally surface) degrees. All of the angular bins were azimuthally symmetric, i.e. there was only one azimuthal angular bin from 0 to 360 degrees. Excluding neutrons and a few photopeaks in the photon plots, the structure in these figures, particularly for the protons and muons, represent Monte Carlo statistical uncertainty in the tally results. Finally, the particle spectra tallied and plotted in Figures A.3 through A.8, and used as step 2 sources, were deemed important based on MCNP output (in print table 126). This table in the MCNP output shows how many particles entered each cell in the geometry. Neutrons, photons, electrons, protons, muons, and anti-muons had the largest populations of particles entering the street.

Integrating the curves in Figures A.3 through A.8 and combining all the particles entering each face produces the total number of each particle type entering the modeled street per second, which is the source strength for each particle type in step 2 of this analysis. These total source strengths are plotted in Figure A.9. 


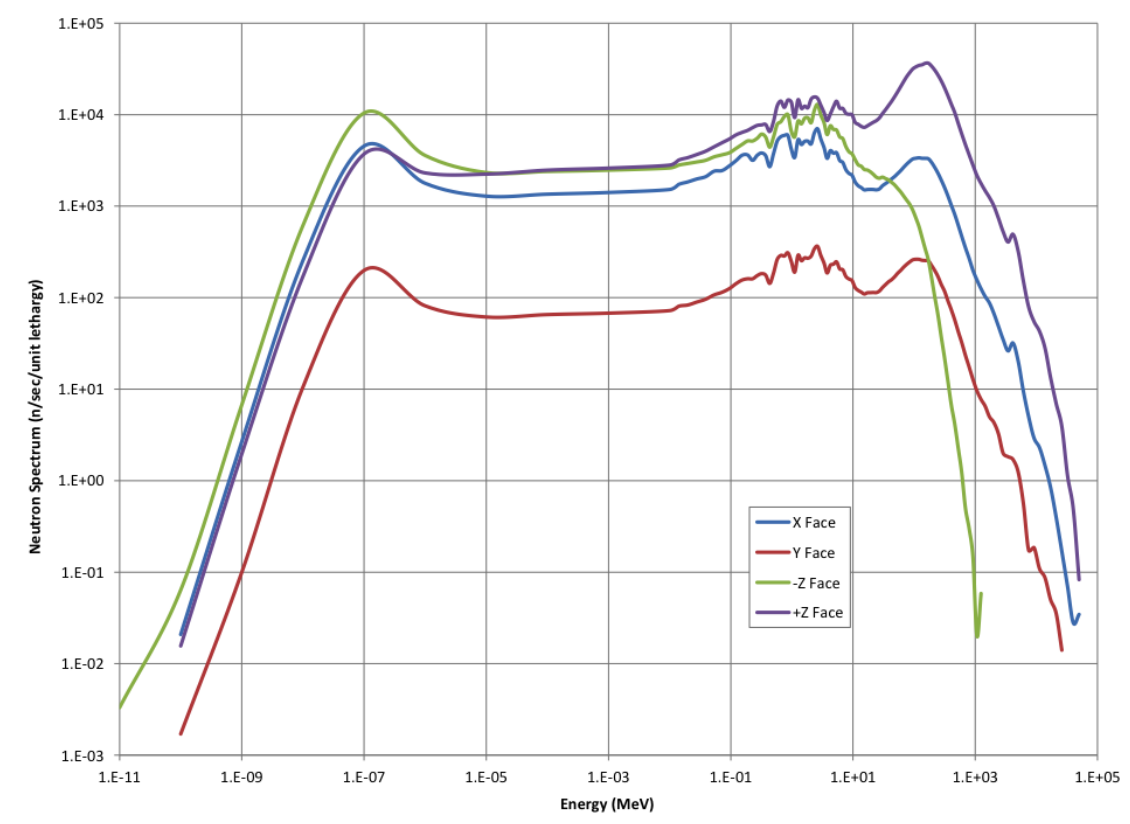

Fig. A.3. Ground level cosmic neutron sources for city block model.

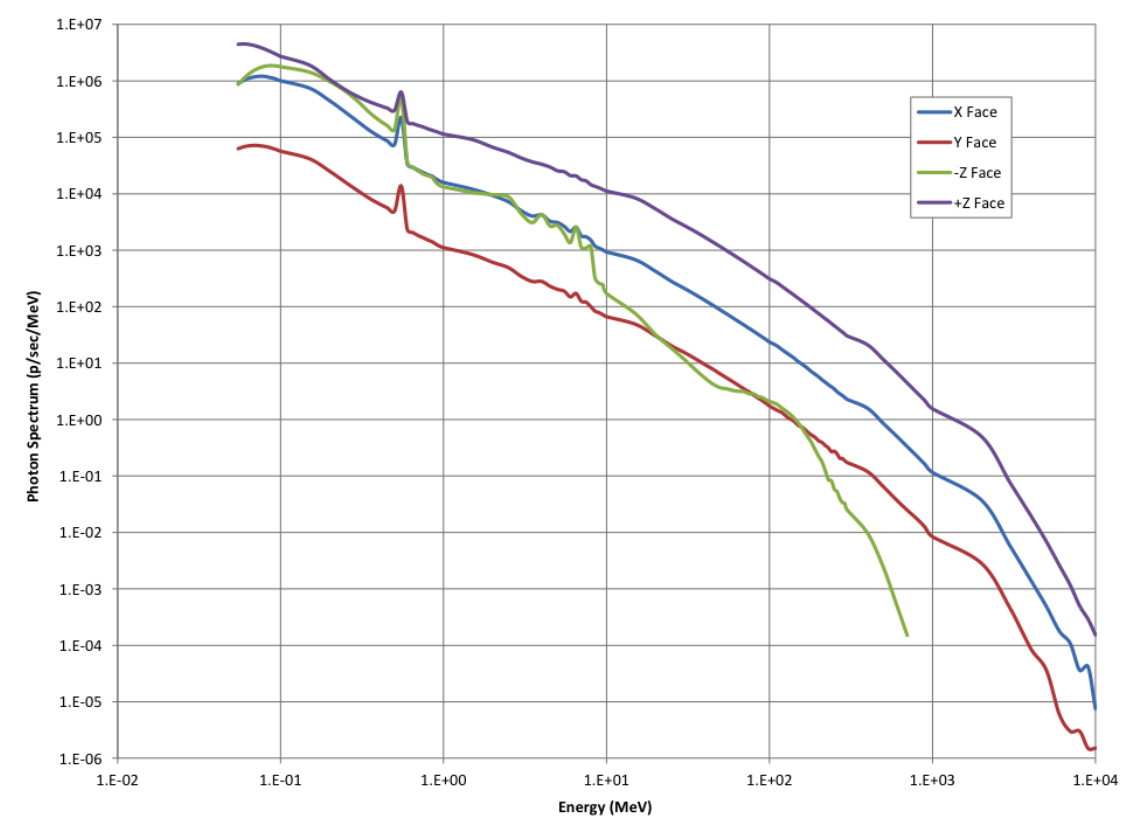

Fig. A.4. Ground level cosmic photon sources for city block model. 


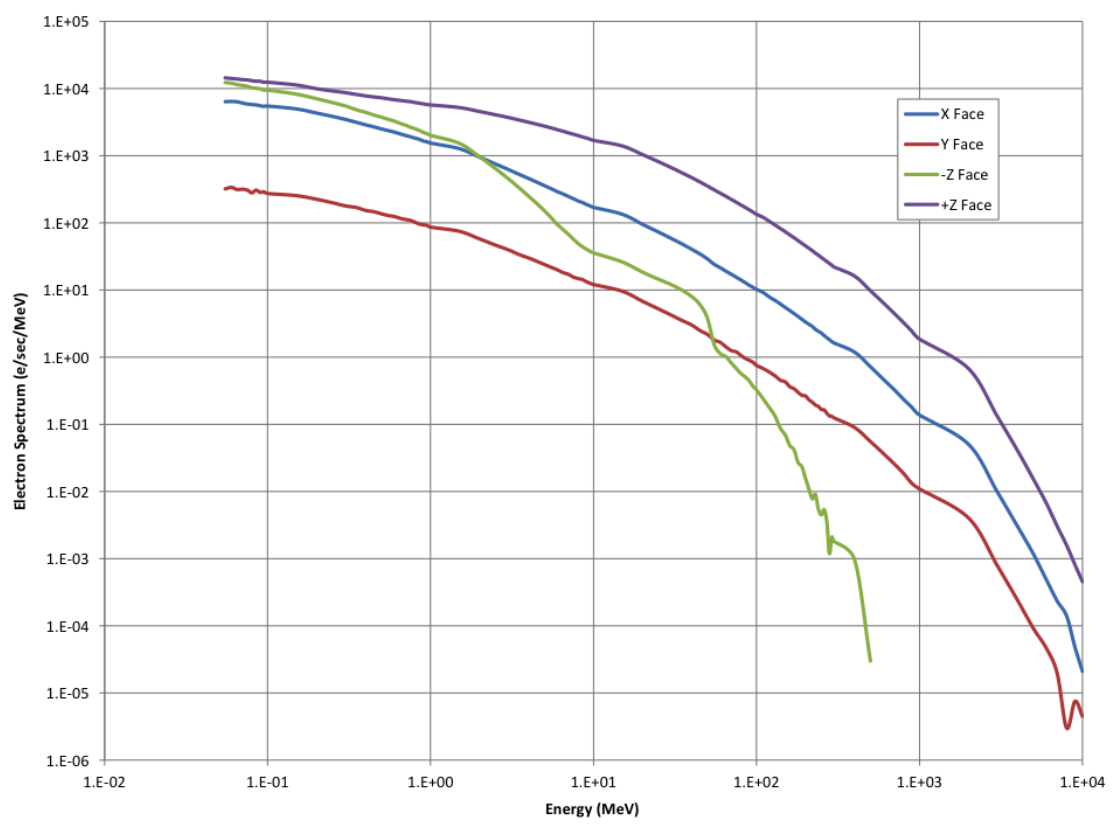

Fig. A.5. Ground level cosmic electron sources for city block model.

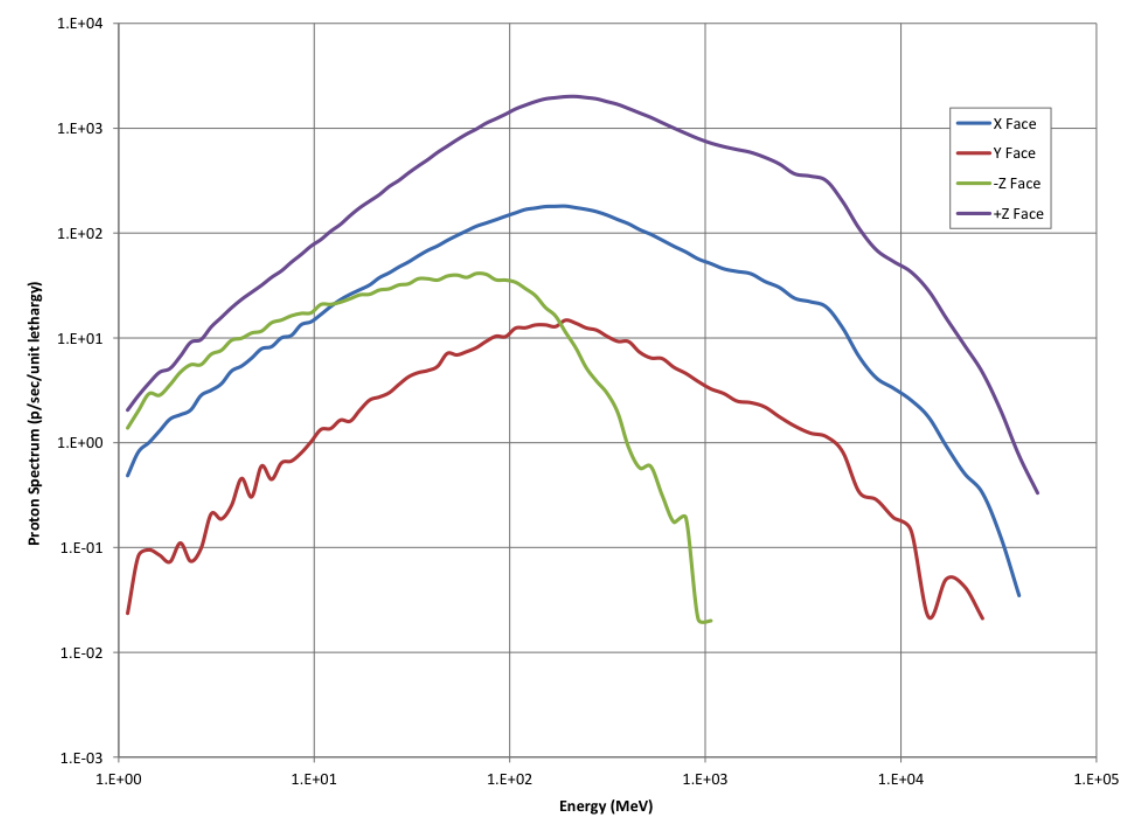

Fig. A.6. Ground level cosmic proton sources for city block model. 


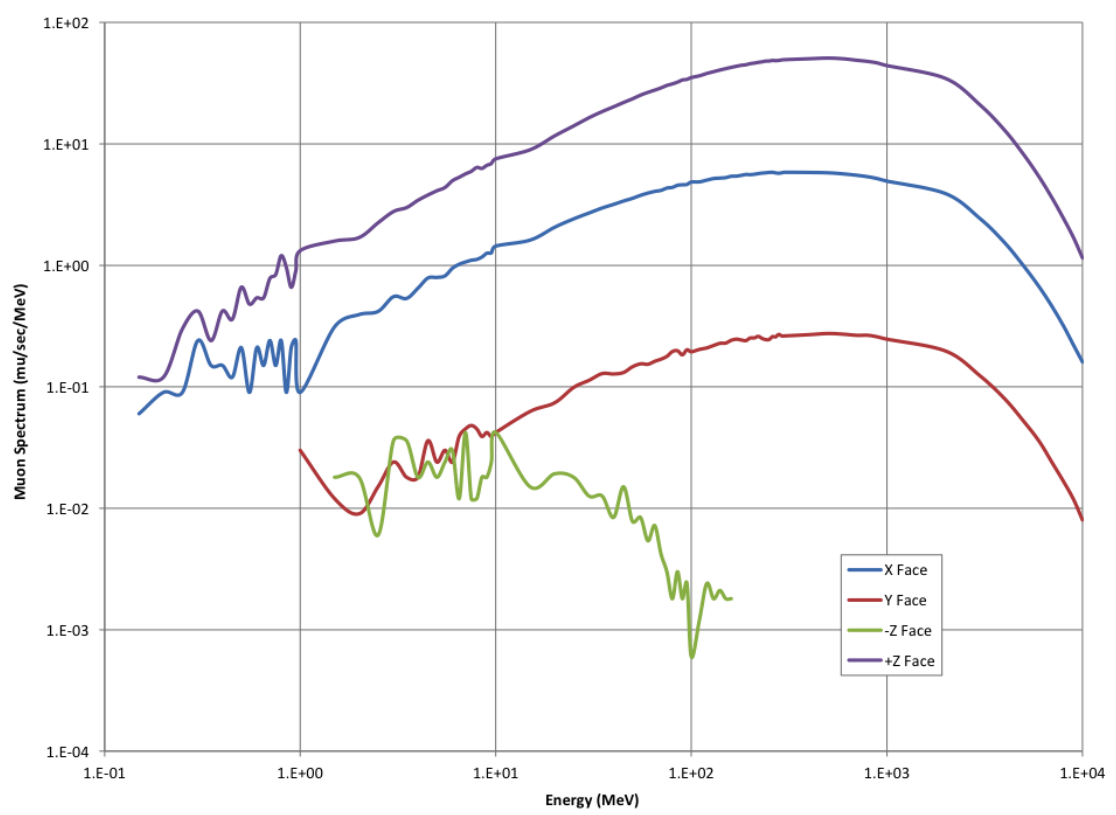

Fig. A.7. Ground level cosmic muon sources for city block model.

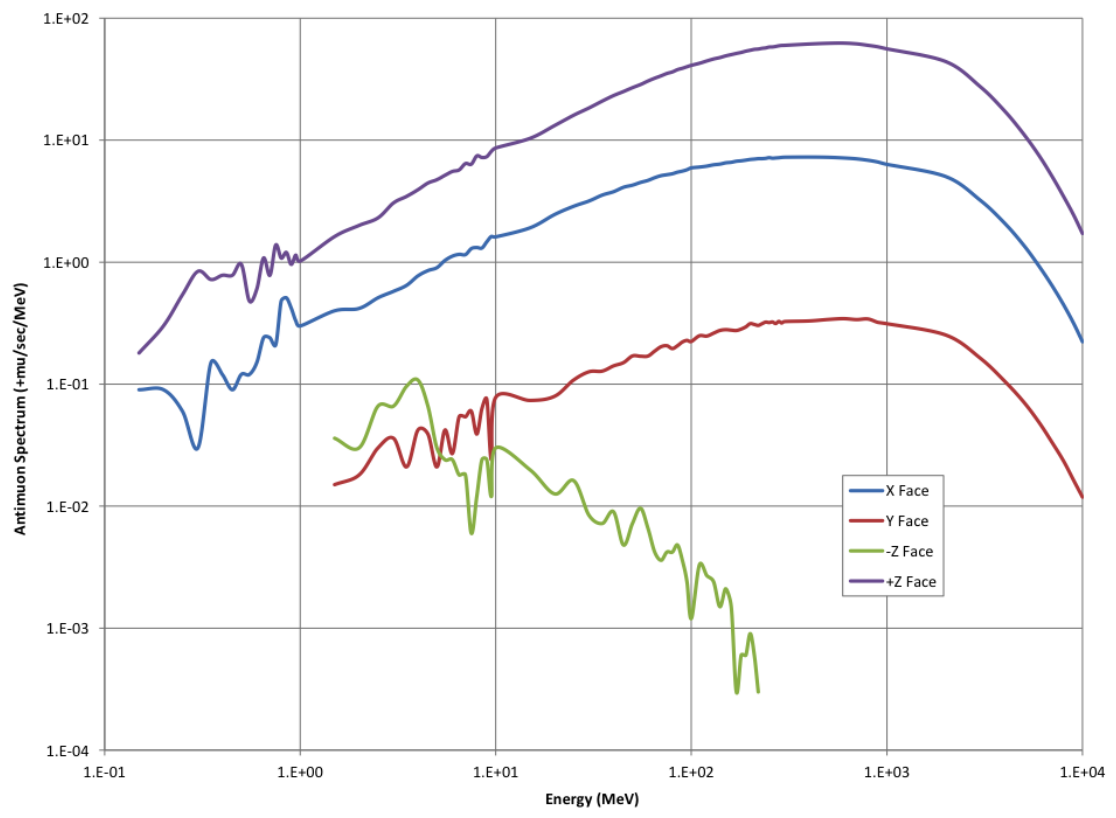

Fig. A.8. Ground level cosmic anti-muon sources for city block model. 


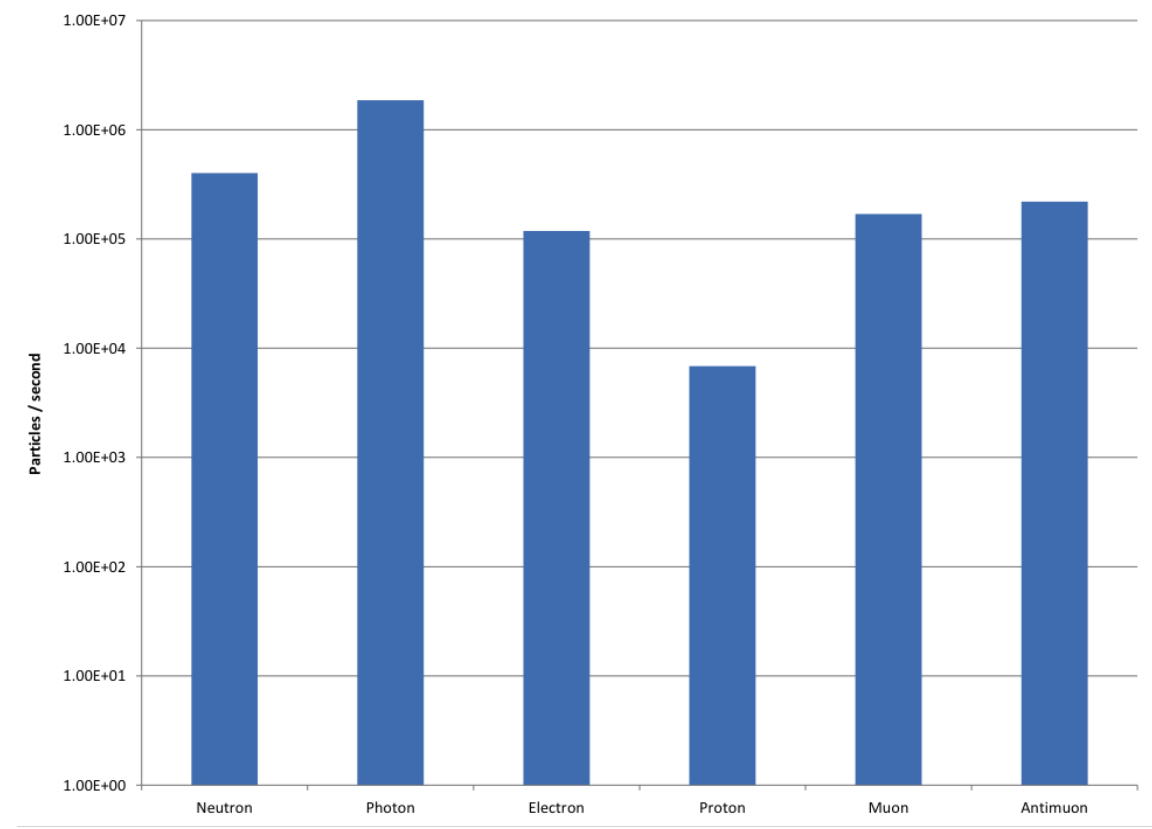

Fig. A.9. Total source strengths for step 2 by particle type.

\section{A.4 STEP 2 RESULTS}

With the sources generated in step 1, the purpose of step 2 is to calculate the photon count rate in a $2 " \times 4$ " $\times 16$ " NaI detector traveling in a car within the city block model. As in step 1, the geometry in Figure A. 2 was modified to create the step 2 model. In step 2 the parked cars along the side of the street and the car with the NaI detector is included in the model. Furthermore, everything outside of the black rectangular parallelepiped in Figure A. 2 has been removed from the step 2 model. These parts were removed because most of the particle interactions outside the rectangular parallelepiped have already been accounted for in the step 1 tally / step 2 source. However, since the cars were not included in the step 1 model and since the street, sidewalk, building faces, and atmosphere are not included in the step 2 model, there are some interaction combinations that are not accounted for in this methodology. Particles that interact with a car in the street, leave the rectangular parallelepiped in Figure A.2, and then return to the rectangular parallelepiped after interacting with the street, sidewalk, building faces, or atmosphere are missing. This missing portion of the signal is a second order effect or smaller.

Recall that the assumption at the outset of this analysis is the GCR induced photon background is small compared to the terrestrial photon background. Therefore, instead of calculating the background spectra at many points along the length of the street, only the background count rate in the NaI located at the center of the street, near the hidden source location, was calculated. The thought being that if the GCR induced photon background count rate is small compared to the terrestrial photon background count rate then the GCR induced photon background can be ignored. Another implicit assumption is that the GCR induced photon background is fairly constant along the street. The count rates simulated in step 2 are actually the average count rate along the street because the sources in step 2 (which are equivalent to the tallies in step 1) represent the average of particles entering the rectangular parallelepiped along the entire street of the city block model.

The photon count rate simulations in step 2 modeled the behavior of a NaI detector with 1024 channels 
that are each $3 \mathrm{keV}$ wide, so the possible detection window is from 0 to $3072 \mathrm{keV}$. However, the detection window is actually smaller because a $50 \mathrm{keV}$ cut off has been applied, so the simulated detection window is actually from 50 to $3072 \mathrm{keV}$. Gaussian energy broadening parameters for a small NaI detector have been applied via the MCNP F8 special tally treatment. The derivation of these parameters is discussed in [4].

Figure A.10 shows the total photon count rate in the NaI detector as a function of source particle type, i.e. particle type entering the street.

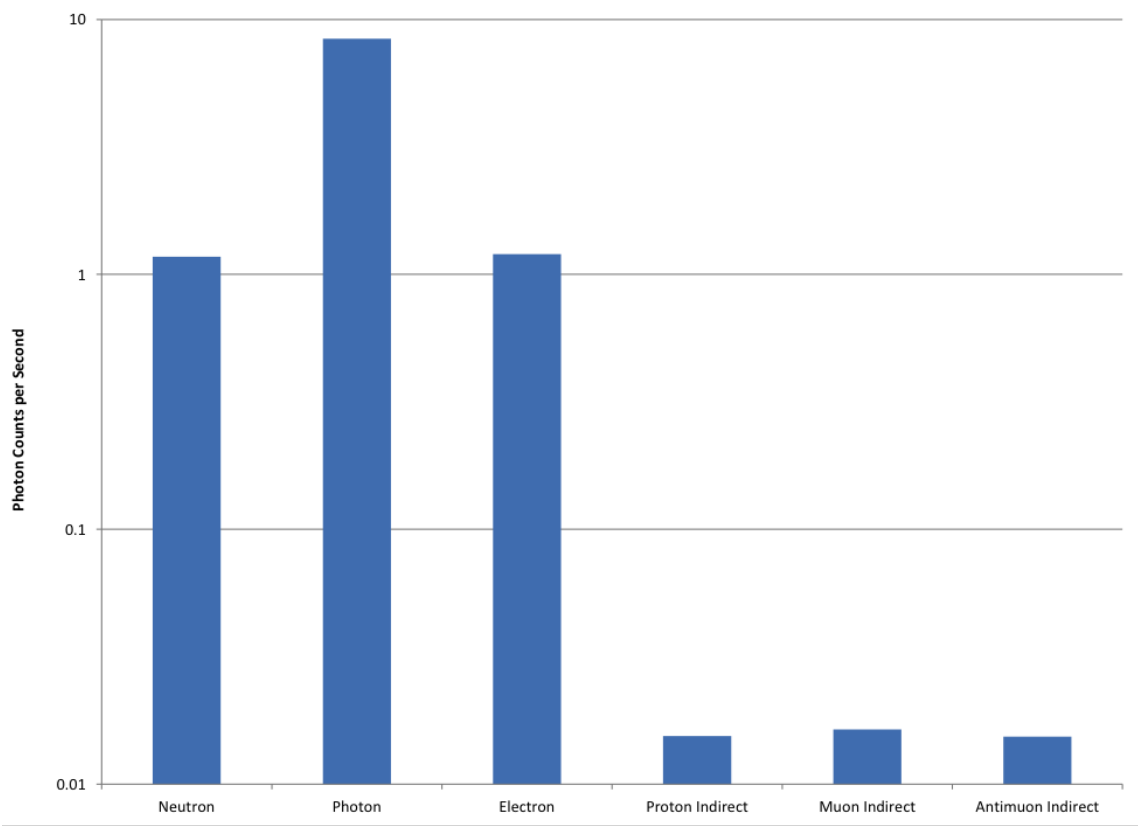

Fig. A.10. Total photon count rates, by source particle type, from 50 to $3072 \mathrm{keV}$. Count rates include energy deposition by photons and electrons only. Direct energy deposition by muons and protons are not included. Indirect contributions from muons and protons are included through decay daughters, and secondary photons and electrons produced by muon and proton collisions.

The step 1 tallies separate the particles entering the street by particle type and face of the rectangular parallelepiped in Figure A.2. Therefore, the information in Figure A.10 can be broken down further into the location of the source particles contributing to the count rates. This information is reported in Table A.2 on a percentage basis to 2 significant figures, so contributions less than $0.01 \%$ are reported as zero. The "Location" notation in Table A.2 refers to Figure A.2, i.e. left in Table A.2 means source particles entering the left face of the rectangular parallelepiped in Figure A.2. Note that the street in the city block model is a one-way street because all the cars in Figure A.2 are parked facing the same direction. The car carrying the $\mathrm{NaI}$ detector is in the left-hand lane of Figure A.2, so the detector is closer to the buildings on the left of Figure A.2. Finally, the street is about $280 \mathrm{~m}$ long and the detector for this simulation is in the middle of this length of street. Therefore, the sources on the front and back of the geometry are very far away from the detector. 


\begin{tabular}{|c|c|c|c|c|c|c|c|}
\hline \hline Location & Neutron & Photon & Electron & Proton & Muon & anti-muon & Total \\
\hline Right & $7.50 \%$ & $7.29 \%$ & $2.91 \%$ & $2.55 \%$ & $0.51 \%$ & $0.49 \%$ & $6.80 \%$ \\
\hline Front & $0.0 \%$ & $0.0 \%$ & $0.0 \%$ & $0.0 \%$ & $0.0 \%$ & $0.0 \%$ & $0.0 \%$ \\
\hline Bottom & $43.21 \%$ & $37.72 \%$ & $1.47 \%$ & $0.47 \%$ & $0.0 \%$ & $0.0 \%$ & $34.13 \%$ \\
\hline Left & $14.41 \%$ & $12.91 \%$ & $6.56 \%$ & $5.93 \%$ & $5.96 \%$ & $4.97 \%$ & $12.33 \%$ \\
\hline Back & $0.0 \%$ & $0.0 \%$ & $0.0 \%$ & $0.0 \%$ & $0.0 \%$ & $0.0 \%$ & $0.0 \%$ \\
\hline Top & $34.88 \%$ & $42.08 \%$ & $89.06 \%$ & $91.05 \%$ & $93.53 \%$ & $94.53 \%$ & $46.73 \%$ \\
\hline Total & $100.0 \%$ & $100.0 \%$ & $100.0 \%$ & $100.0 \%$ & $100.0 \%$ & $100.0 \%$ & $100.0 \%$ \\
\hline \hline
\end{tabular}

Table A.2. Directional properties of GCR induced photon background

\section{A.5 CONCLUSIONS}

The grand total of photon counts plotted in Figure A.10 is about 10.8 counts/sec, which has a Monte Carlo uncertainty of about 1\%. Earlier comparisons of simulations to Goldhagen's measurements of GCR induced neutron background had differences of $+/-40 \%$ [5]. Therefore, this is a reasonable guess at the bias between these city block simulations and actual measurements (this is a major assumption). Data presented in reference [6] show the variations in the calculated GCR induced photon flux on the surface of Earth due to parameters like the solar cycle, location, weather, and surface composition are $10 \%$ or less. Note this specifically excludes elevation, which was a very large effect (factor 10 for a $3000 \mathrm{~m}$ change in elevation). Adding all these uncertainties in quadrature, i.e. assume everything is uncorrelated, gives a total uncertainty of about $41 \%$. Therefore, a conservative estimate of the GCR induced photon background along the street of the city block model is approximately 20 counts/sec, on the $95 \%$ confidence interval. The calculated terrestrial photon background count rates along the street of the city block model vary between 1500 and 2000 counts/sec, so the estimated GCR induced photon background is less than a few percent of the modeled terrestrial photon background. While these specific count rates are only applicable to this model, it is believed that the GCR induced photon background at sea level will typically be less than $5 \%$ of the terrestrial photon background and likely may be ignored during operational modeling. Possible exceptions include situations with very low KUT content surrounding the detector (like at sea), at high elevations, and during a very large solar particle event (which are rare and often damage electronics).

\section{References}

[1] R. J. McConn et al., "Compendium of Material Composition Data for Radiation Transport Modeling," PNNL-15870 Rev. 1, Pacific Northwest National Laboratory (March 4, 2011).

[2] J. M. Picone et al., "NRL-MSISE-00 Empirical Model of the Atmosphere: Statistical Comparisons and Scientific Issues,” J. Geophys. Res., Vol. 107, Iss. A12 (2002).

[3] R. C. Singleterry et al., "OLTARIS: On-Line Tool for the Assessment of Radiation in Space," NASA/TP-2010-216722, Langley Research Center, Hampton, Virginia (2010).

[4] H.H. Hakimabad, "Response Function of a $3 \times 3$ in. NaI Scintillation Detector int eh range of 0.081 to 4.438 MeV”, Asian J. Exp. Sci., Vol. 21, No. 2, 2007, 233-237.

[5] T. M. Miller, W. C. de Wet, and B. W. Patton, "Computational Assessment of Naturally Occurring Background Radiation Produced by Extraterrestrial Sources," 18th Topical Meeting of the Radiation 
Protection and Shielding Division of the American Nuclear Society, Knoxville, Tennessee, USA, September 2014.

[6] A. Nicholson, D. E. Hornback, B W. Patton, T. M. Miller, et. al., "Systematic Assessment of Neutron and Gamma-ray Backgrounds Relevant to Operational Modeling and Detection Technology Implementation,” ORNL/LTR-2013/596 (2014). 


\section{B COMPUTATIONAL STUDIES WITH A SIMPLIFIED MODEL OF GAY STREET}

To better understand the contributions of different background sources to the total background count rate, several studies were done using a very simplified representation of the 400 block of Gay Street in Knoxville, TN. This block is a typical urban street with a variety of building types and sizes. Each building face, street and sidewalk was modeled as a simple rectangular block with different concentrations of ${ }^{40} \mathrm{~K}$, ${ }^{238} \mathrm{U}$ and ${ }^{232} \mathrm{Th}$.

\section{B.1 MODELING}

\section{B.1.1 Geometry}

Figure B.1 shows the geometry of the model and Table B.1 lists the modeled buildings. This block is $565 \mathrm{ft}(17221.2 \mathrm{~cm})$ long and has brick or concrete buildings up to six stories tall. Buildings were modeled as nested rectangular boxes, uniformly 6 in $(15.25 \mathrm{~cm})$ thick, without doors or windows and extending $10 \mathrm{ft}$ $(304.8 \mathrm{~cm})$ below street level. The model version of Gay Street is $40 \mathrm{ft}(1219.2 \mathrm{~cm})$ wide with $12 \mathrm{ft}(365.76$ $\mathrm{cm})$ sidewalks on each side. Building faces along the street are all $12 \mathrm{ft}(365.76 \mathrm{~cm})$ from the edge of the street. The top of the sidewalks define the $\mathrm{z}=0$ plane and are 6 in $(15.25 \mathrm{~cm})$ thick. The base of the model is concrete extending $15 \mathrm{ft}(475.2 \mathrm{~cm})$ below the top of the sidewalk. The surfaces of the streets are 6 in $(15.24 \mathrm{~cm})$ below the tops of the sidewalks and contain inlaid bricks near intersections. The layout and distances were based on imagery from Google Earth.

Table B.1. The buildings along Gay Street (addresses go north to south)

\begin{tabular}{cccc}
\hline \hline West Side & & & East Side \\
\hline & Wall Avenue & 402 & Mast General Store \\
401 & Shorty & 408 & House \\
403 & Lerner Lofts & 412 & Black Roof \\
411 & 411 Building & 418 & Phoenix \\
413 & Baker's & 422 & Art Market \\
415 & Skybox & 424 & Woodruff's \\
417 & Kress Building & 428 & Sapphire \\
431 & KUB & 430 & Slomski Taylor \\
465 & Miller's Building & & Union Avenue \\
& Union Avenue & 502 & 502 (The Market) \\
& & 504 & Alley Cover \\
\hline \hline
\end{tabular}

\section{B.1.2 Sources}

Three natural sources considered were 1) ${ }^{40} \mathrm{~K}, 2$ ) a uranium mixture (called ${ }^{238} \mathrm{U}$ in this report) that contains the ${ }^{238} U$ decay chain and the ${ }^{235} U$ decay chain, both in secular equilibrium with their daughters, with the ratio of the uranium isotopes that corresponds to their natural abundances, and 3) the ${ }^{232} \mathrm{Th}$ decay chain in secular equilibrium with its daughters. The three sources were scaled to $1 \mathrm{~Bq}$ of the lead isotope. In the case of the uranium, the activity of the ${ }^{238} \mathrm{U}$ was scaled to $1 \mathrm{~Bq}$ because that is typically what is measured and reported for different materials. The SCALE/ORIGEN code [1] was used to compute the gamma emission spectrum and the total number of gammas per decay for the three source terms. The 


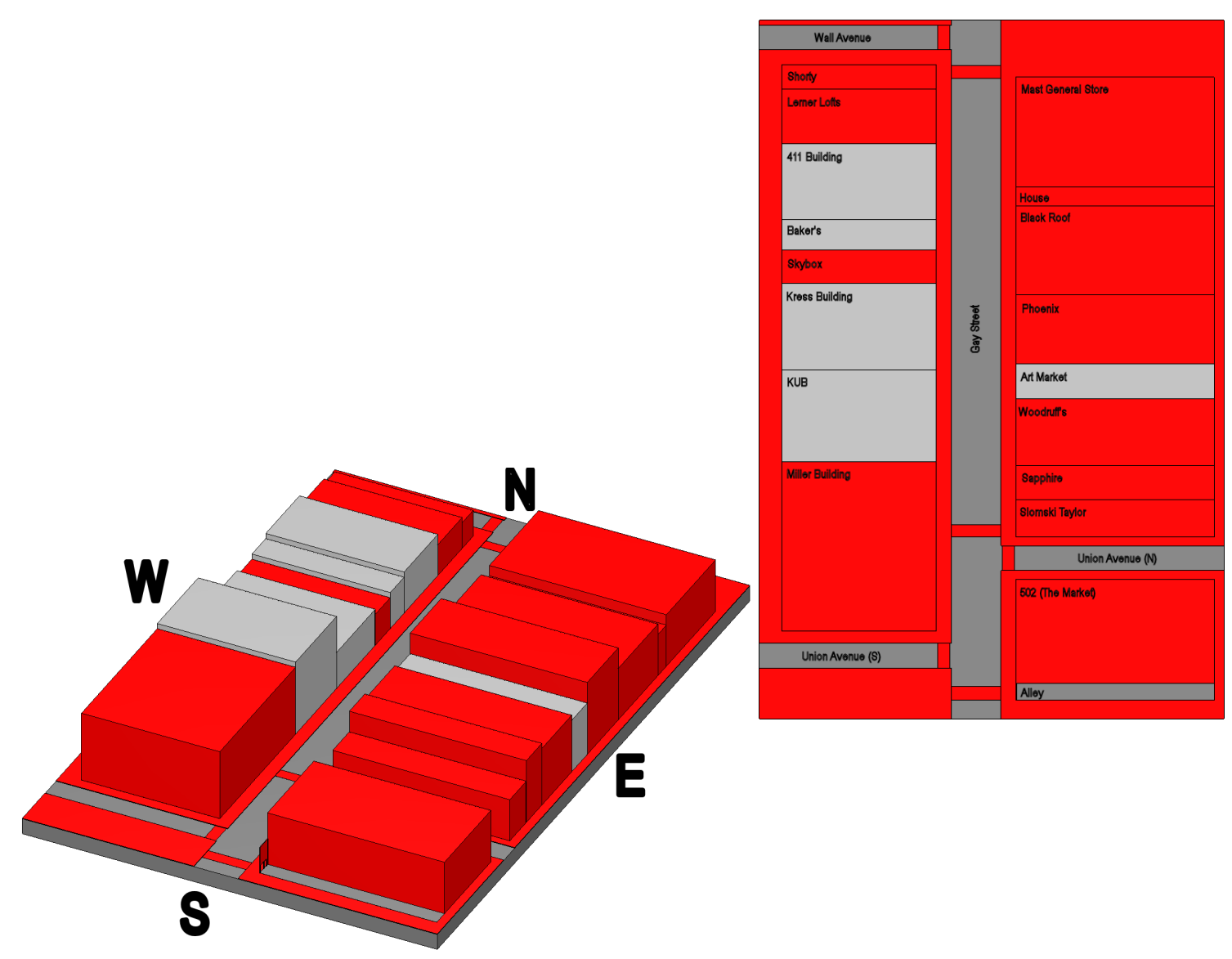

Fig. B.1. Model geometry of the 400 block of Gay Street. Materials are brick (red), granite (light gray) and concrete (dark gray). 

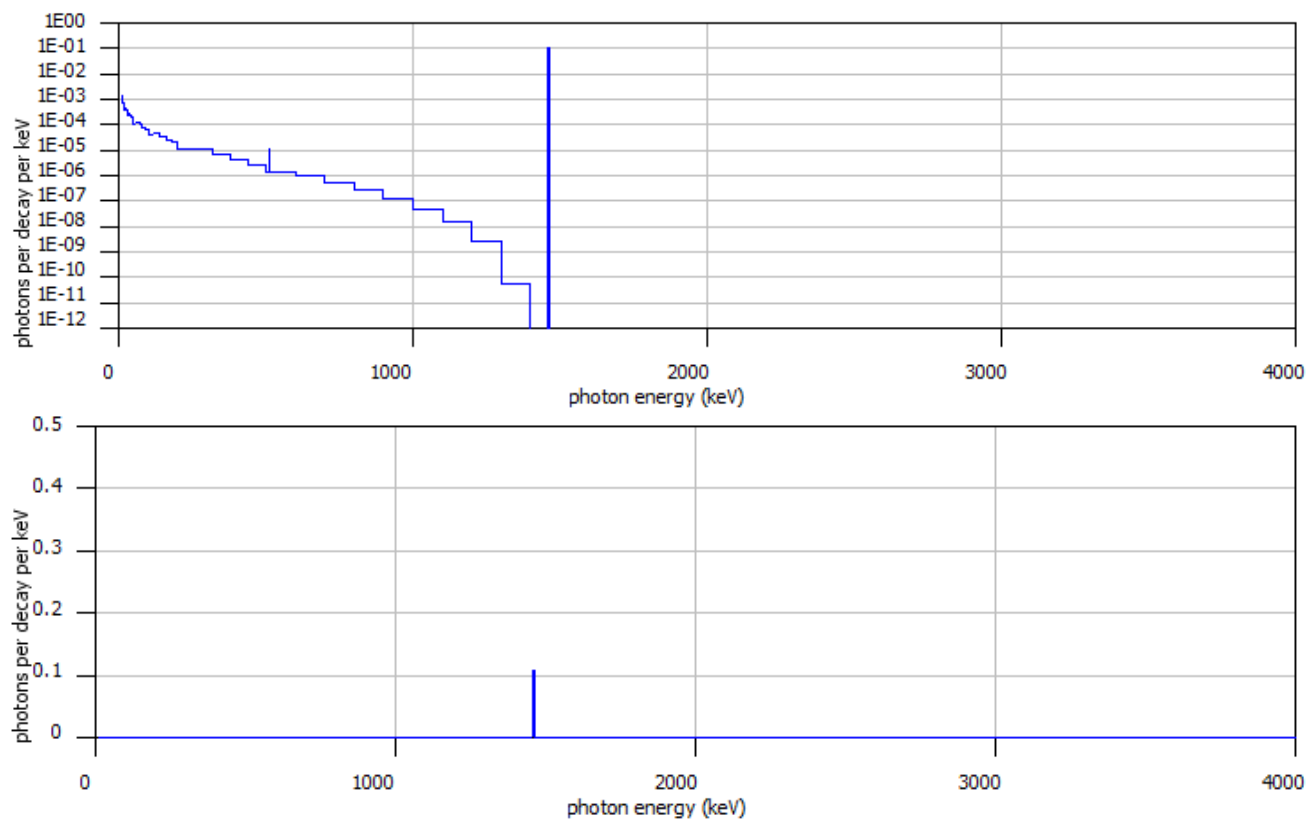

Fig. B.2. Gamma emission probabilities for the ${ }^{40} \mathrm{~K}$ source - 1Bq (0. 1344 gammas/sec). Both logarithmic scale (top) and linear scale (bottom) are shown.
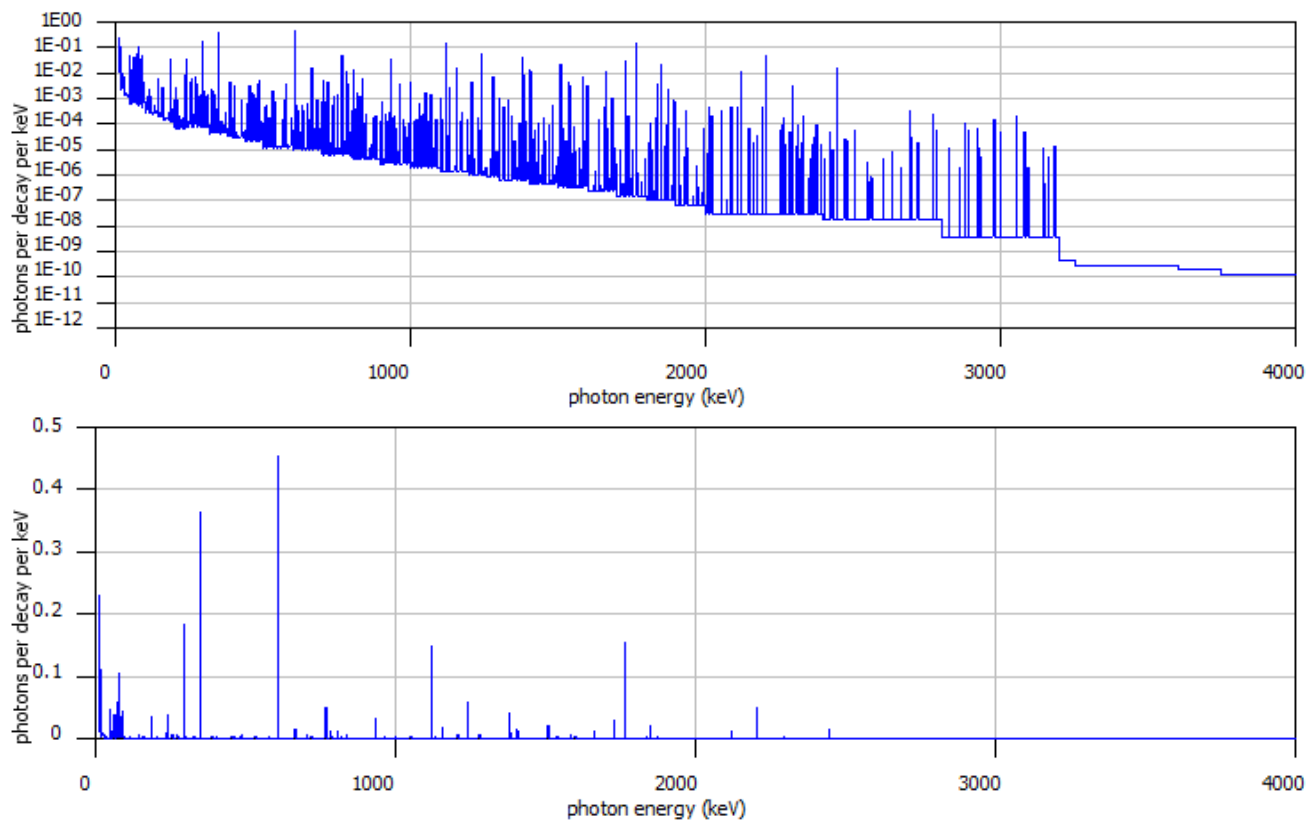

Fig. B.3. Gamma emission probabilities for the U source - 1Bq (3.4725 gammas/sec). Both logarithmic scale (top) and linear scale (bottom) are shown. 
Table B.2. Data used to determine the starting masses for constructing the gamma emission spectra with SCALE/ORIGEN

\begin{tabular}{cccccc}
\hline \hline & ${ }^{40} \mathrm{~K}$ & ${ }^{232} \mathrm{Th}$ & ${ }^{235} \mathrm{U}$ & ${ }^{238} \mathrm{U}$ & data source \\
\hline $\begin{array}{c}\text { atomic mass } \\
\text { abundance }\end{array}$ & 40 & 232 & 235 & 238 & ORIGEN data \\
half-life $(\mathrm{sec})$ & $3.938400 \mathrm{E}+16$ & $4.433800 \mathrm{E}+17$ & $2.221000 \mathrm{E}+16$ & $1.410000 \mathrm{E}+17$ & $\begin{array}{c}\text { ORIGEN data } \\
\text { Chart of Nuclides }\end{array}$ \\
decay const $(/ \mathrm{sec})$ & $1.759972 \mathrm{E}-17$ & $1.563325 \mathrm{E}-18$ & $3.120879 \mathrm{E}-17$ & $4.915937 \mathrm{E}-18$ & $\ln (2) / t_{\frac{1}{2}}$ \\
mass $(\mathrm{g})$ & $3.774013 \mathrm{E}-06$ & $2.464267 \mathrm{E}-04$ & & $8.039327 \mathrm{E}-05$ & for 1 Bq today \\
mass $(\mathrm{g})$ & & & $5.757121 \mathrm{E}-07$ & & based on U-238 \\
decay (seconds) & $1.410000 \mathrm{E}+17$ & $1.410000 \mathrm{E}+17$ & $1.410000 \mathrm{E}+17$ & $1.410000 \mathrm{E}+17$ & age of earth \\
starting mass $(\mathrm{g})$ & $4.513684 \mathrm{E}-05$ & $3.071982 \mathrm{E}-04$ & $4.691285 \mathrm{E}-05$ & $1.607865 \mathrm{E}-04$ & \\
\hline \hline
\end{tabular}

Table B.3. Activity densities (Bq/kg) used in the model

\begin{tabular}{cccc}
\hline \hline & ${ }^{40} \mathrm{~K}$ & ${ }^{238} \mathrm{U}$ & ${ }^{232} \mathrm{Th}$ \\
\hline brick & $550,650,750$ & $40,50,60$ & $40,50,60$ \\
concrete & $250,300,350$ & $30,40,50$ & $30,40,50$ \\
granite & $1000,1200,1400$ & $50,60,70$ & $40,50,60$ \\
\hline \hline
\end{tabular}

masses and other parameters used to construct the ORIGEN input are listed in Table B.2. The computed gamma emission spectra for the three sources are shown in Figs. B.2-B.4.

The amounts of ${ }^{40} \mathrm{~K},{ }^{238} \mathrm{U}$ and ${ }^{232} \mathrm{Th}$ were assigned to the buildings (only the fronts facing Gay Street), the sidewalks along Gay Street, the streets and the brick inlays. All sources were 6 in $(15.24 \mathrm{~cm})$ thick. Mean values for the nuclide concentrations were chosen based on the values in the literature [2] [3]. Buildings were randomly assigned one of three values for a particular material/isotope combination. A summary of values used in the model is given in Table B.3. The values assigned to the buildings, streets and sidewalks in the model are listed in Table B.4. For the building faces, the overall source strengths were reduced by a factor of two to partially account for the windows and doors.

\section{B.2 DETECTOR RESPONSE FUNCTION}

An approach commonly used in complex source/detector simulations is to use a detector response function (DRF) [4]. A detailed simulation of a detector is done to determine its response (probability of depositing energy or simple interaction rate) as a function of different incoming particle energies. Using a DRF allows the calculation of the small-scale detector interactions to be separated from the large-scale transport calculations. The large-scale transport calculation can compute the energy-dependent flux in many locations where a detector might be placed and then simply convolve those fluxes with the DRF to compute the detector response if the detector had been modeled in those locations.

One item that is not accounted for in the DRF approach compared to a simulation that includes the detector in the large-scale transport calculation is the small chance that a particle could interact with the detector, leave, scatter on surrounding materials and then re-enter the detector. In most cases, this difference is small compared to other modeling uncertainties (true composition of materials, true density of materials, etc). To minimize this effect, it is important to include the entire detector system in the detailed 

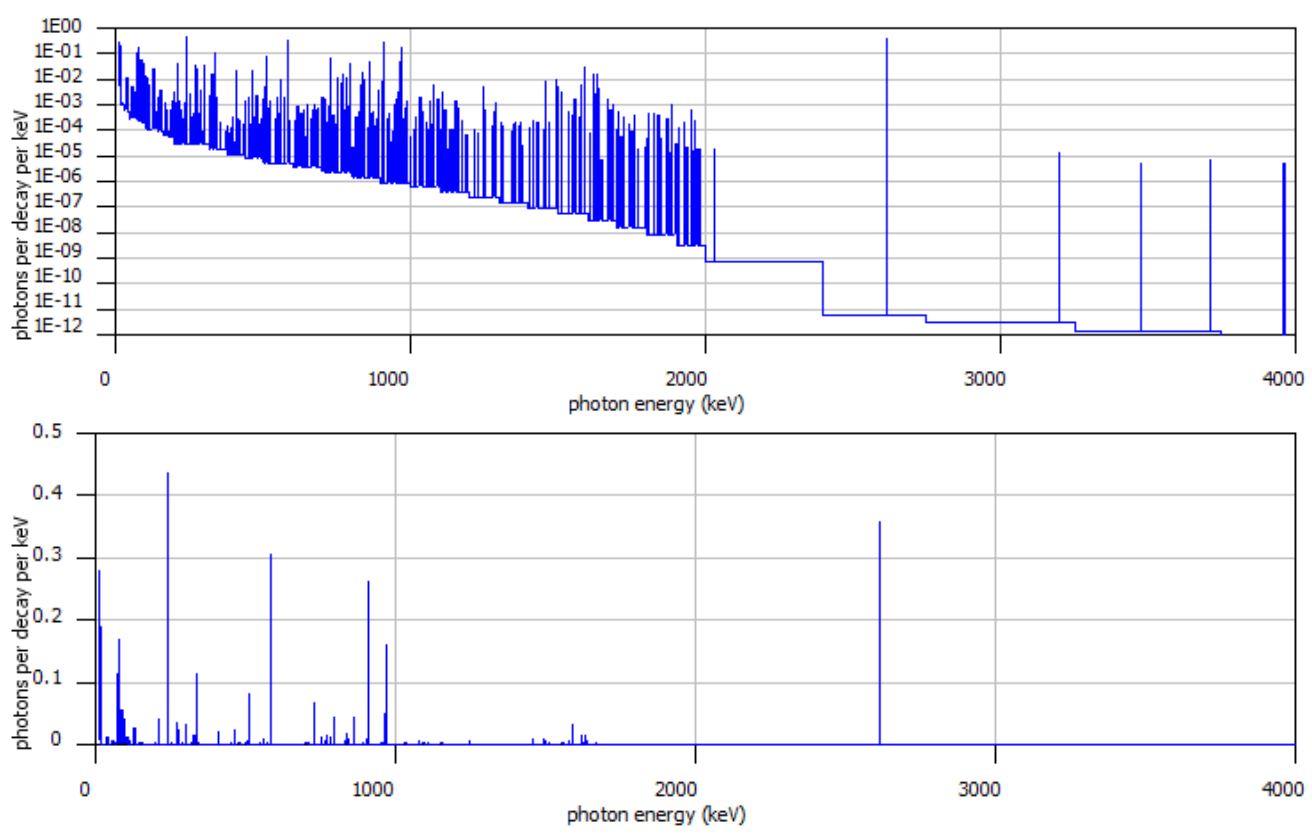

Fig. B.4. Gamma emission probabilities for the ${ }^{232}$ Th source - 1Bq (3.9974 gammas/sec). Both logarithmic scale (top) and linear scale (bottom) are shown.

calculation of the DRF (i.e. everything that moves with the detector). In an urban search scenario, this would include the person wearing the backpack or the vehicle containing the detector.

For most detectors, the direction of the incoming particle is an important parameter in the response. This is more important when using an energy-deposited distribution than when looking only at detector count rate. To minimize the difference between a complete transport simulation and the DRF approach, the DRF should be calculated with an incident particle distribution similar to that which would be seen in the complete transport simulation. For example, for a detector hung below a helicopter that is used to examine the background sources from the ground, the DRF should be found by using particles starting over a single large plane below the detector. For a detector measuring the background inside a concrete building, the DRF should be found for particles coming in from all directions.

For the simplified Gay Street model, a DRF was calculated for a $2 \times 4 \times 16$ inch $(5.08 \times 10.16 \times 40.64 \mathrm{~cm})$ $\mathrm{NaI}$ detector with a $1 \mathrm{~mm}$ thick aluminum cover using MCNP [5]. For a given incident energy, photons were simulated starting on a sphere of radius $\mathrm{R}=25 \mathrm{~cm}$ surrounding the detector model, heading into the sphere. The source strength was set to $\mathrm{R}^{2}$ photons/sec so that the average flux within the sphere without the detector would have been $1 / \mathrm{cm}^{2} / \mathrm{sec}$. A pulse-height tally was used to record the energy deposited in the $\mathrm{NaI}$ material. Depositions of less than $50 \mathrm{keV}$ were not included, to better reflect a real detector with a low-energy cutoff. The final detector response function, which converts a flux $\left(/ \mathrm{cm}^{2} / \mathrm{sec}\right)$ into a count rate (counts/sec), is shown in Fig. B.5 and Table B.6.

\section{B.3 SOURCE IMPORTANCE STUDY}

To gauge the relative importance of the various sources in the model (37 bodies $\times 3$ isotopic chains) to the count rate that would be measured in the middle of Gay Street, an adjoint calculation was done using SCALE/MAVRIC [6] and Denovo [7]. The adjoint source used the response function as the energy 


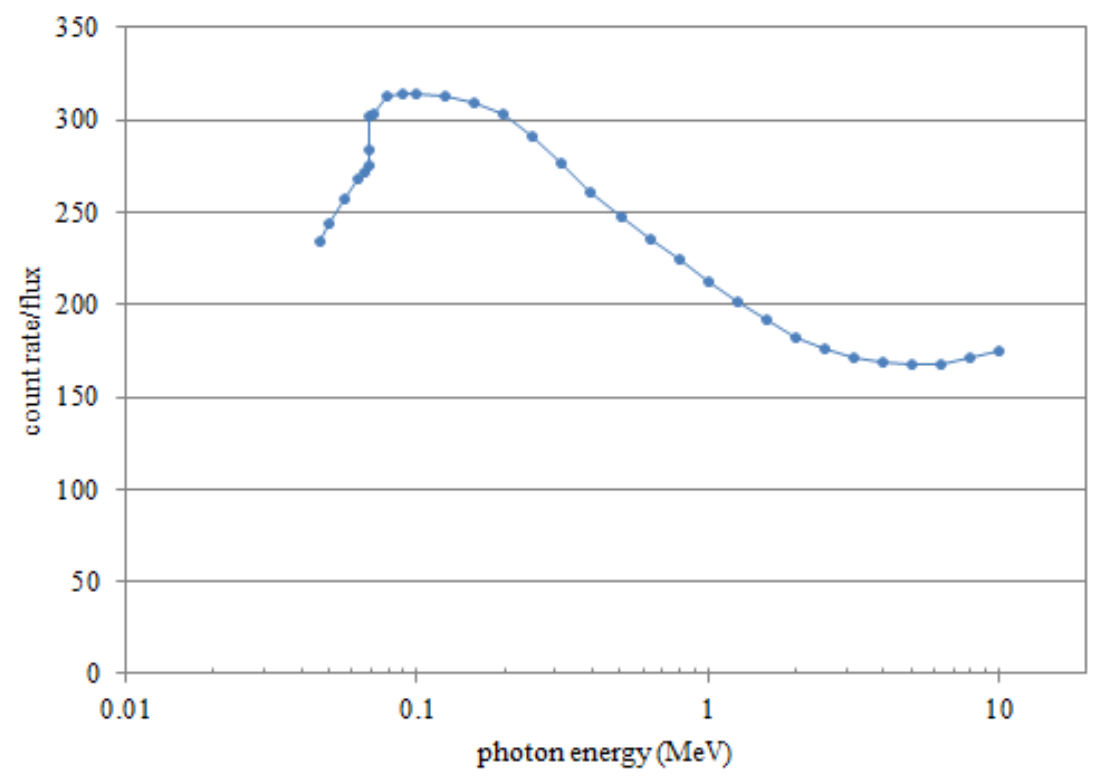

Fig. B.5. Computed detector response function ([count $\left./ \mathrm{sec}] /\left[1 / \mathrm{cm}^{2} / \mathrm{sec}\right]\right)$ for a $2 \times 4 \times 16$ inch NaI detector with a $1 \mathrm{~mm}$ thick aluminum cover

distribution at a location corresponding to the center of the north-bound lane near the midpoint of the 400 block of Gay Street.

The adjoint fluxes were computed over the entire model and combined with the three source emission distributions. Figure B.6 shows a canyon view of the model where the sources are the street, the sidewalks and the building faces along the street. The importances of the sources will be shown for the floor and walls of this canyon (where the background sources would actually be). To better display the results, the importances will be shown for a flattened view of this canyon, which is also shown in Fig. B.6.

Figures B.7-B.9 show the relative importance for the three different source types along the Gay Street canyon. The plots cover four orders of magnitude, with a factor of $\sqrt[3]{10} \approx 2.15$ for each color contour. The importance for the potassium is for $100 \mathrm{~Bq}$ because its activity densities are typically much higher than uranium and thorium and so that the three figures can all use the same scale. All three plots show the same thing-that the street directly under the detector is about 10-20 times more important than the nearest point of the closest buildings. Qualitatively it is known that the importance of a source location should fall off with the square of the distance from the detector but these figures quantitatively show the importance of a type of source that includes its gamma energy emission distribution and the detector response function. Note that these importance values do not depend on the source densities assigned in the model - these plots show the importance of a source $\left(100 \mathrm{~Bq}\right.$ of ${ }^{40} \mathrm{~K}, 1 \mathrm{~Bq}$ of ${ }^{238} \mathrm{U}$ or $\left.1 \mathrm{~Bq}^{232} \mathrm{Th}\right)$ to contributing to the detector count rate.

The adjoint fluxes can also be combined with the different source volumes and the source amounts to determine the contribution of the sources as assigned in the model. These relative contributions were computed for the 37 bodies and 3 isotopic chains and are listed in Table 6, sorted from highest contributor to lowest contributor. The rankings of the sources is somewhat expected since the street under the detector and two closest buildings on each side of the road lead the contributions to the total count rate. The amounts listed in the table show that the street itself is responsible for almost $44 \%$ of the count rate; the 

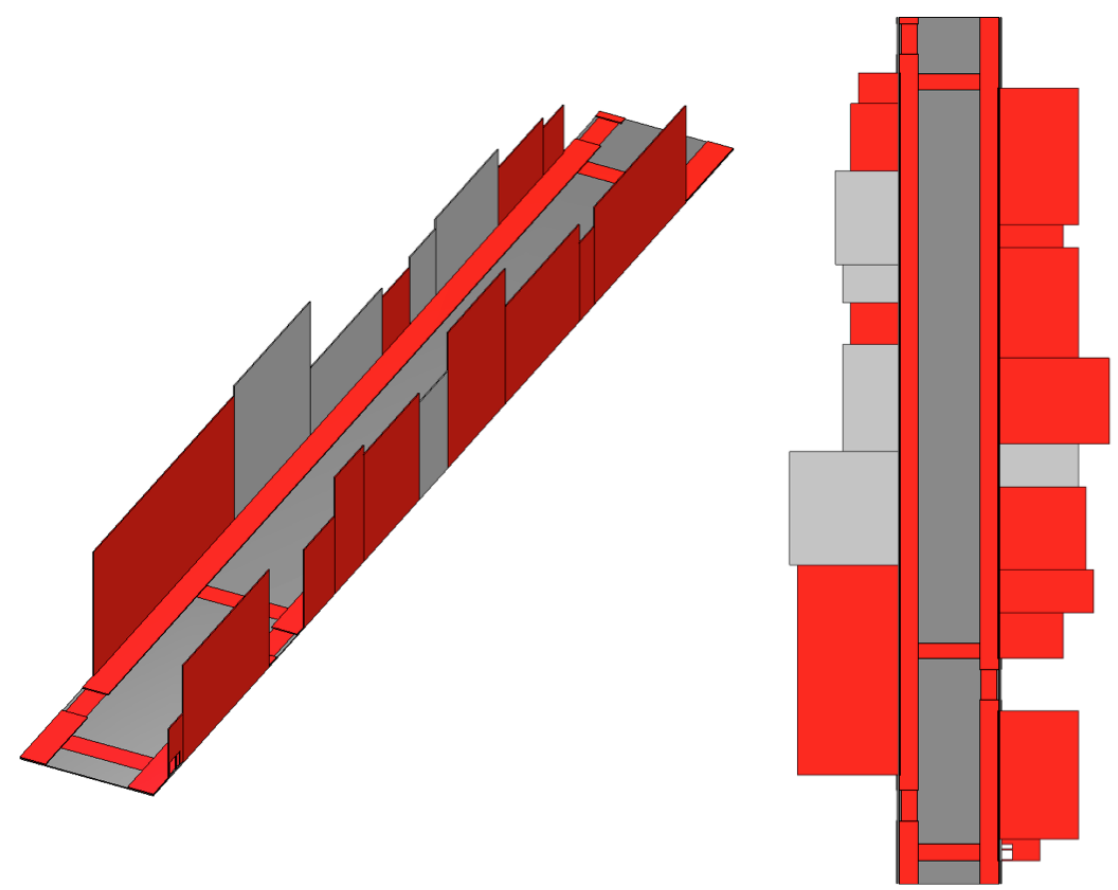

Fig. B.6. (Left) Urban canyon consisting of the main street, sidewalks and building faces. (Right) The canyon laid out flat - a basis for interpreting latter plots.

street, the two closest buildings and the two sidewalks combined contribute more than $80 \%$; adding the next four closest buildings accounts for $95 \%$ of the total count rate. The relative contributions for this detector location from ${ }^{40} \mathrm{~K} /{ }^{238} \mathrm{U} /{ }^{232} \mathrm{Th}$ were computed to be $22 / 41 / 37$. Monte Carlo calculations for this detector location show the contributions to be $24 / 39 / 37$.

The source importance plots (for unit sources) and the table listing the relative contributions from the source strengths assigned in this simple model both show that the background count rate is only a function of the sources that a very close to the detector and because of that, could vary quite quickly as the detector location changes.

\section{B.4 DETECTOR COUNT RATE ALONG GAY STREET STUDY}

MAVRIC and the FW-CADIS hybrid method [8] were used to compute the detector count rate all along Gay Street. Denovo calculations for computing the importance map used a non-uniform mesh grid of $84 \times 234 \times 122=2398032$ cells, a QR quadrature with 4 polars per octant and 4 azimuths per octant, and a P3 Legendre expansion of the scattering. The forward Denovo required 5.3 hours and the adjoint Denovo (using the detector response function as the adjoint source, $150 \mathrm{~cm}$ above the street) required 5 hours. Monte Carlo calculations for K, U, and Th sources were computed separately and each required 12 hours to get less than $1.5 \%$ relative uncertainties in the street for a mesh tally with uniform grid of $30.48 \mathrm{~cm} \times 30.48$ $\mathrm{cm} \times 60.96 \mathrm{~cm}$.

Figure B.10 shows the count rates above $150 \mathrm{~cm}$ Gay Street for the three components of background. The scale is adjusted to highlight the range of values near the center of the street where measurements can be taken in a vehicle. Higher count rates close to building faces that are clipped in the ${ }^{232} \mathrm{Th}$ plot extend up 


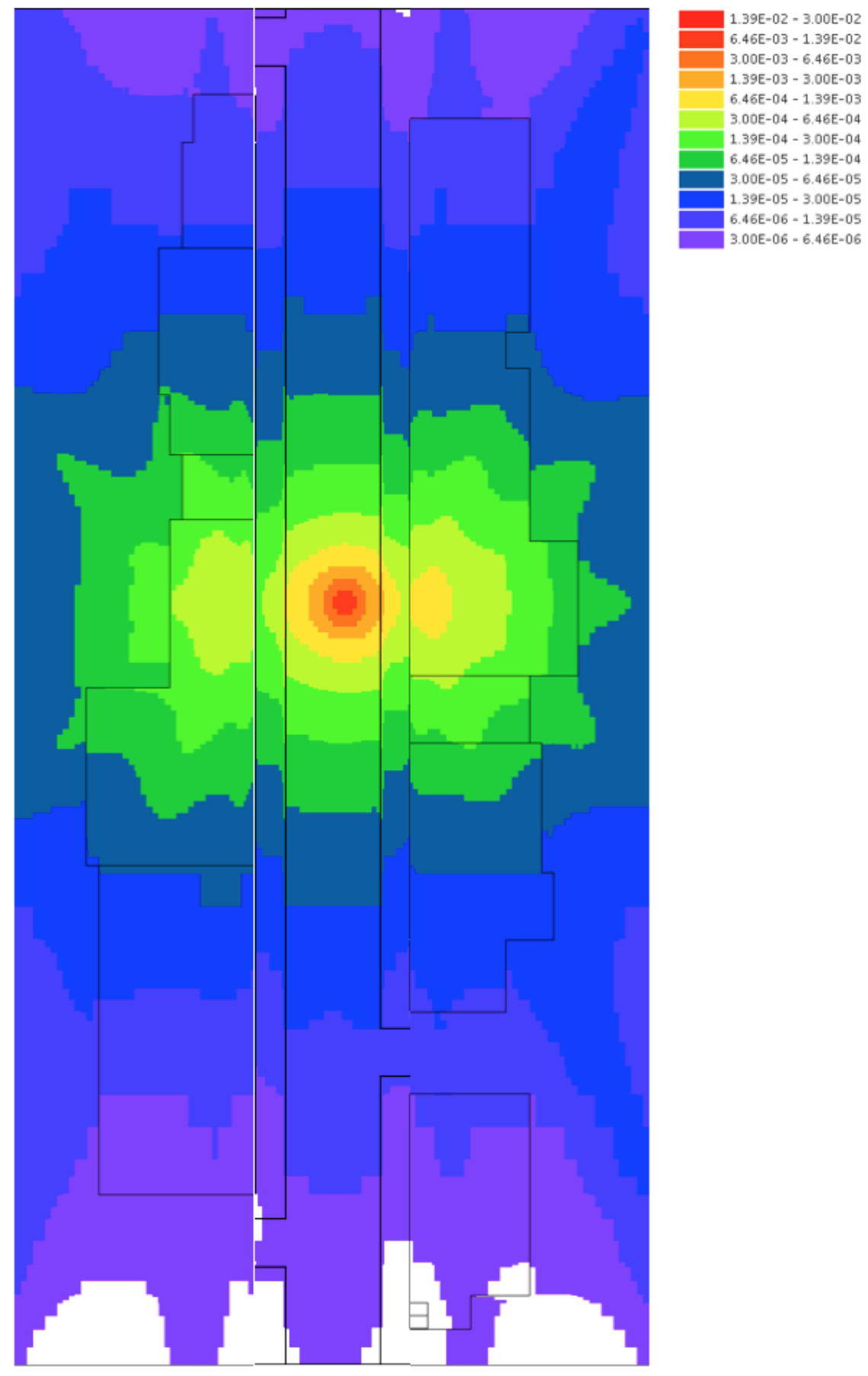

Fig. B.7. Importance for a $100 \mathrm{~Bq}$ K-40 source using a canyon view. 


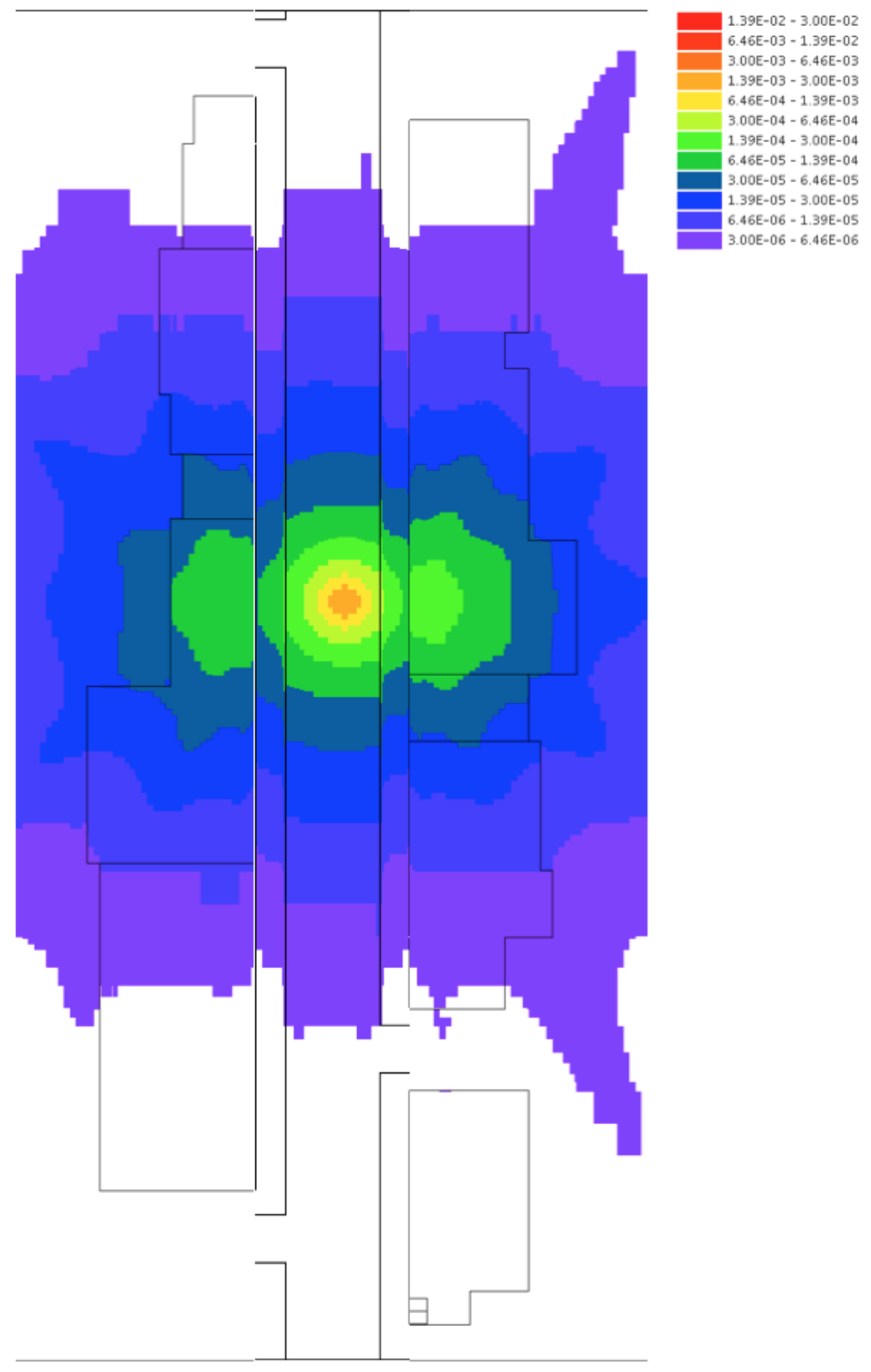

Fig. B.8. Importance for a $100 \mathrm{~Bq}$ U238 source using a canyon view. 


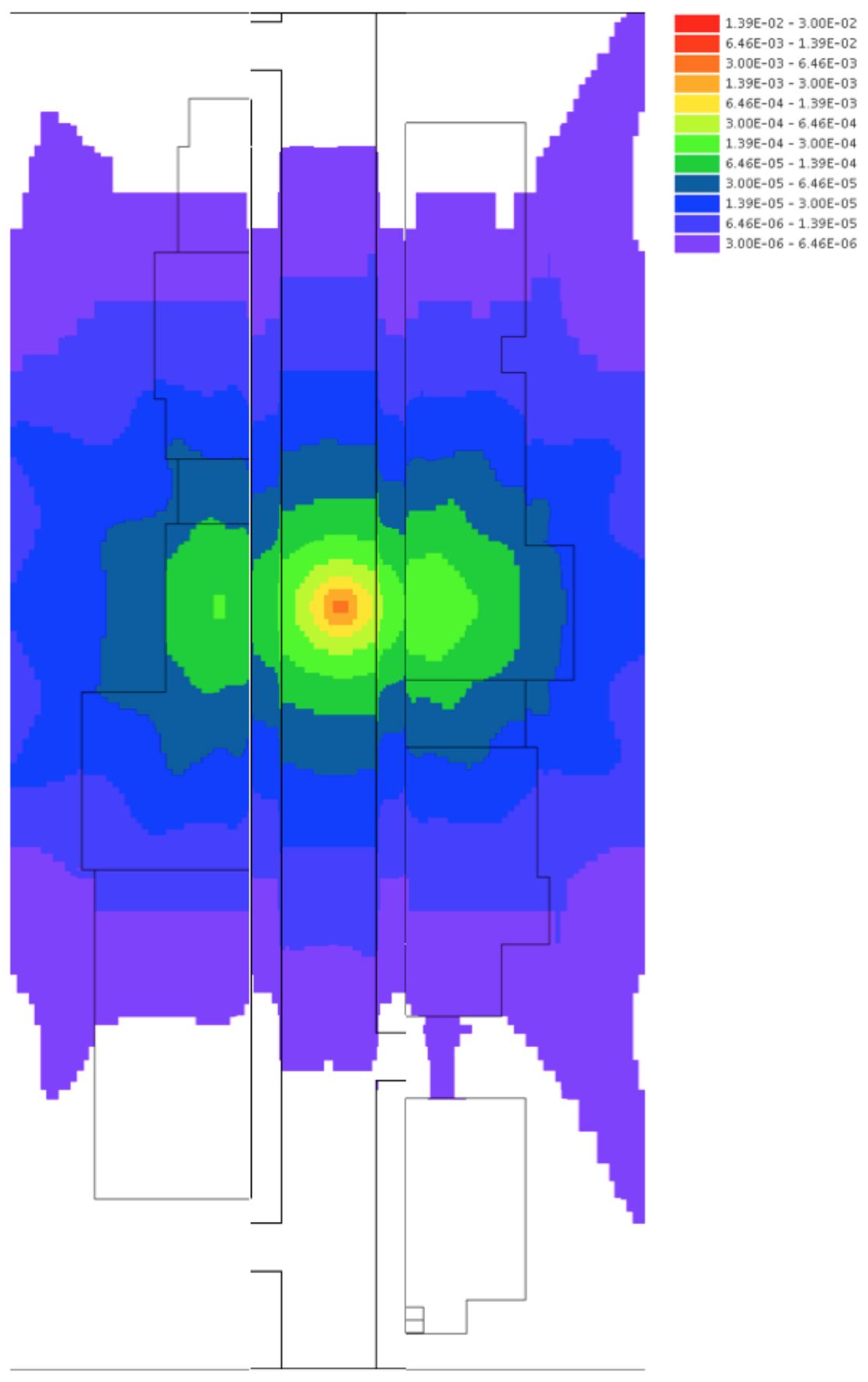

Fig. B.9. Importance for a $100 \mathrm{~Bq}^{232} \mathrm{Th}$ source using a canyon view. 

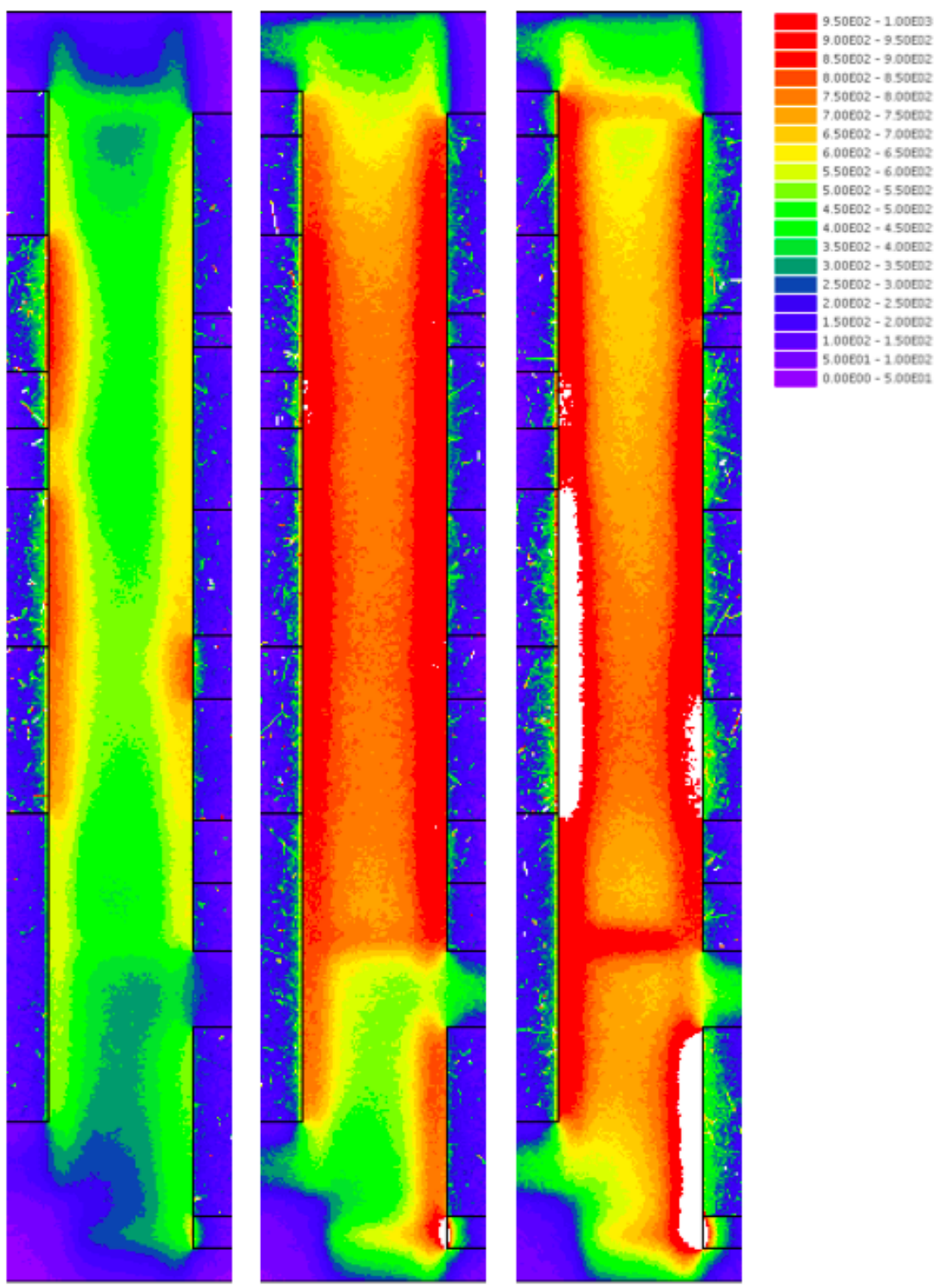

Fig. B.10. Count rates (counts/sec) in Gay Street from ${ }^{40} \mathbf{K}$ (left), ${ }^{238} \mathbf{U}$ (center), ${ }^{232} \mathbf{T h}$ (right). Relative uncertainties were $2 \%$ in the street 

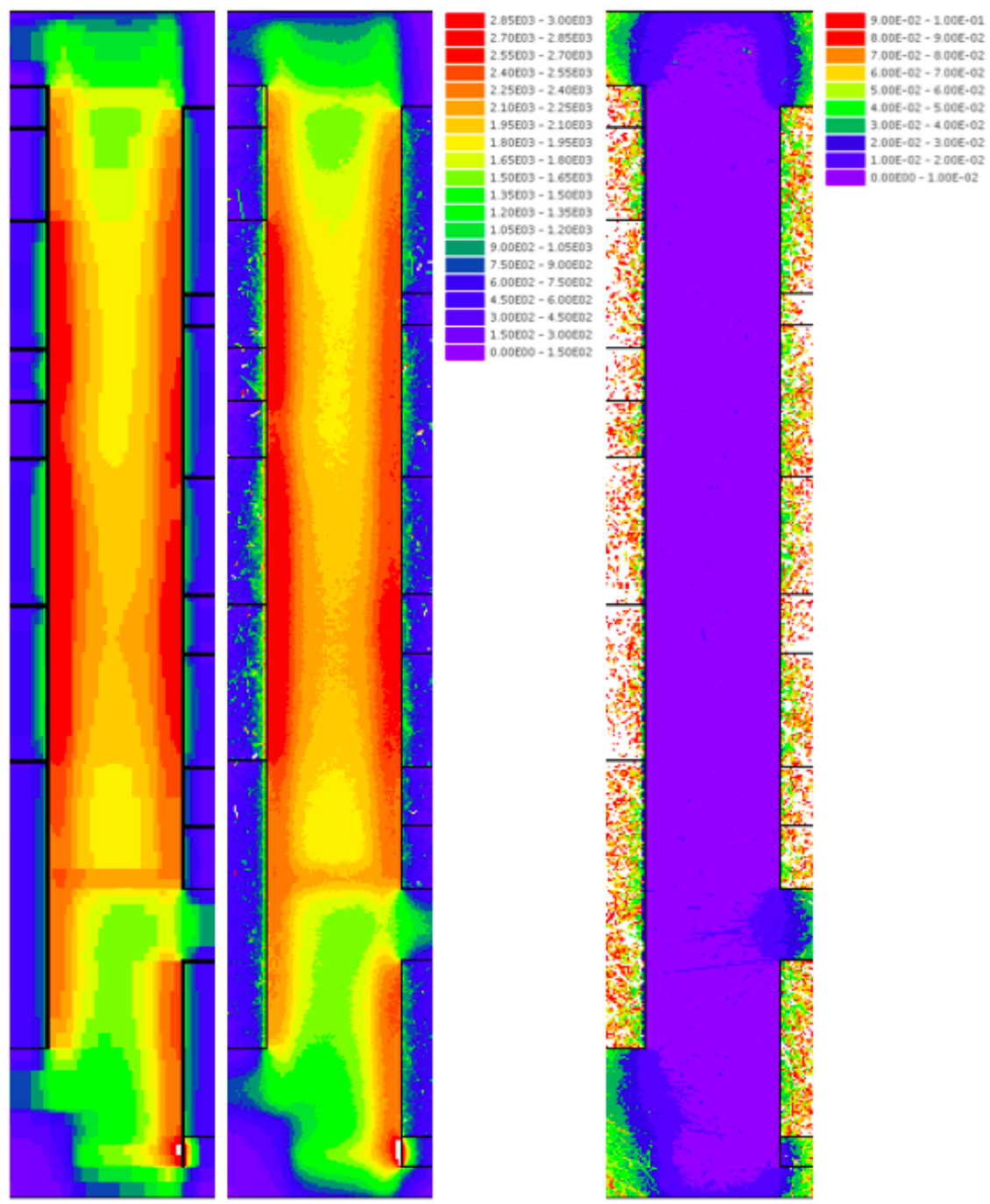

Fig. B.11. Total detector count rates (counts/sec) from Denovo (left) and MAVRIC (middle). Relative uncertainties for MAVRIC were typically less than $1 \%$ (right). 
to 1500 counts per second. Figure B.11 shows the total count rate along the street from both the forward Denovo calculation and the sum of the three Monte Carlo calculations. The results from the two different calculation methods are quite similar. The uncertainty in the Monte Carlo total is also displayed, showing that the relative uncertainties are below $1 \%$ for most of the street. Relative uncertainties in the buildings are much larger because the importance map was developed to optimize the count rates above the street at the expense of everything else.

Figure B.12 shows the components and the total count rate as a function of distance along Gay Street for the north-bound traffic. Figure B.13 shows the relative uncertainties for those count rates. For the activity density values chosen for this simplified model, the ${ }^{232} \mathrm{Th}$ accounts for $20-25 \%$ of the total count rate, the ${ }^{238} \mathrm{U}$ accounts of $30-40 \%$, and the ${ }^{232} \mathrm{Th}$ accounts for $35-45 \%$. The count rates vary along the length of Gay Street due to the different activity loadings in the buildings and the brick inlays in the street. This study shows that in order to accurately model the background count rate, the sources need to be well known.

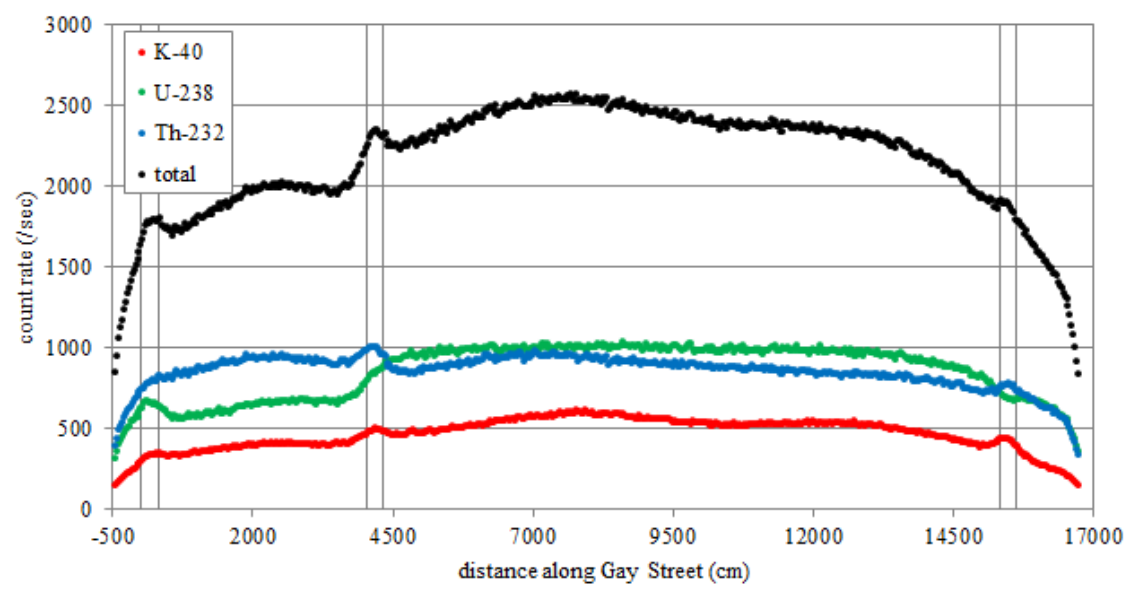

Fig. B.12. Count rates (counts/sec) along the center of the north-bound lane of Gay Street. The three sharp peaks correspond to the locations of the brick inlays near intersections.

The hybrid methods used for this study were developed at ORNL. These methods increase the efficiency of the Monte Carlo calculations by an order of magnitude, allowing them to be done in reasonable times with lower statistical uncertainties. 


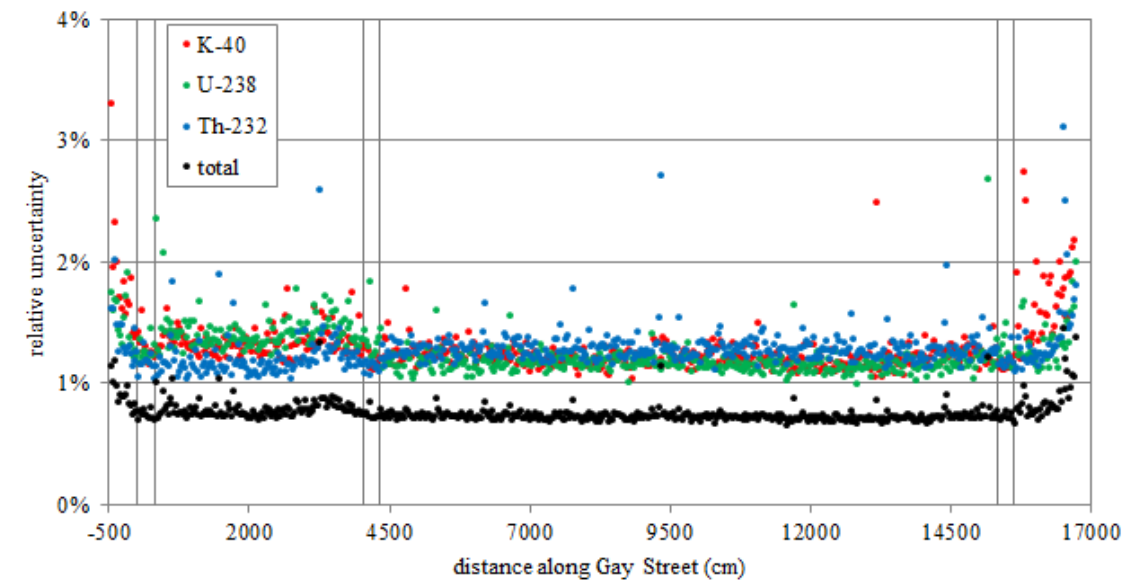

Fig. B.13. Count rate relative uncertainties along the center of the north-bound lane of Gay Street. 
Table B.4. Source strengths in the model

\begin{tabular}{|c|c|c|c|c|c|c|}
\hline emitter & material & $\begin{array}{l}\text { density } \\
(\mathrm{g} / \mathrm{cm} 3)\end{array}$ & $\begin{array}{l}\text { volume } \\
(\mathrm{cm} 3)\end{array}$ & $\begin{array}{c}\text { concentrations }(\mathrm{Bq} / \mathrm{kg}) \\
\mathrm{K}-40\end{array}$ & $\mathrm{U}-238$ & Th-232 \\
\hline Shorty & brick & 1.8 & $7.079 \mathrm{E}+06$ & 650 & 50 & 60 \\
\hline Lerner Lofts & brick & 1.8 & $1.869 \mathrm{E}+07$ & 650 & 50 & 40 \\
\hline 411 Building & granite & 2.7 & $3.455 \mathrm{E}+07$ & 1400 & 60 & 40 \\
\hline Baker's & granite & 2.7 & $1.239 \mathrm{E}+07$ & 1200 & 70 & 50 \\
\hline Skybox & brick & 1.8 & $1.147 \mathrm{E}+07$ & 750 & 60 & 40 \\
\hline Kress Bldg & granite & 2.7 & $3.469 \mathrm{E}+07$ & 1200 & 60 & 60 \\
\hline KUB & granite & 2.7 & $7.334 \mathrm{E}+07$ & 1000 & 50 & 60 \\
\hline Miller's Buillding & brick & 1.8 & $1.261 \mathrm{E}+08$ & 550 & 50 & 40 \\
\hline Mast General Store & brick & 1.8 & $6.300 \mathrm{E}+07$ & 750 & 50 & 60 \\
\hline House & brick & 1.8 & $8.495 \mathrm{E}+06$ & 750 & 60 & 40 \\
\hline Black Roof & brick & 1.8 & $5.097 \mathrm{E}+07$ & 750 & 50 & 60 \\
\hline Phoenix & brick & 1.8 & $5.550 \mathrm{E}+07$ & 750 & 50 & 50 \\
\hline Art Market & granite & 2.7 & $1.982 \mathrm{E}+07$ & 1200 & 50 & 50 \\
\hline Woodruff's & brick & 1.8 & $4.205 \mathrm{E}+07$ & 750 & 40 & 60 \\
\hline Sapphire & brick & 1.8 & $2.379 \mathrm{E}+07$ & 550 & 50 & 40 \\
\hline Slomski Taylor & brick & 1.8 & $1.699 \mathrm{E}+07$ & 750 & 40 & 40 \\
\hline 502 (The Market) & brick & 1.8 & $5.947 \mathrm{E}+07$ & 750 & 40 & 60 \\
\hline Alley Cover & brick & 1.8 & $4.955 \mathrm{E}+06$ & 550 & 50 & 60 \\
\hline Gay street & concrete & 2.3 & $8.495 \mathrm{E}+06$ & 250 & 40 & 40 \\
\hline Gay brick inlay $S$ & brick & 1.8 & $6.230 \mathrm{E}+06$ & 550 & 60 & 50 \\
\hline Gay street & concrete & 2.3 & $6.853 \mathrm{E}+07$ & 300 & 30 & 40 \\
\hline Gay brick inlay & brick & 1.8 & $5.663 \mathrm{E}+06$ & 550 & 50 & 60 \\
\hline Gay street & concrete & 2.3 & $2.044 \mathrm{E}+08$ & 300 & 50 & 30 \\
\hline Gay brick inlay $\mathrm{N}$ & brick & 1.8 & $5.663 \mathrm{E}+06$ & 750 & 40 & 50 \\
\hline Gay street & concrete & 2.3 & $2.095 \mathrm{E}+07$ & 300 & 50 & 40 \\
\hline Union Avenue $S$ & concrete & 2.3 & $4.078 \mathrm{E}+07$ & 250 & 40 & 40 \\
\hline Union $\mathrm{S}$ brick inlay & brick & 1.8 & $2.832 \mathrm{E}+06$ & 650 & 50 & 40 \\
\hline Union Avenue $\mathrm{S}$ & concrete & 2.3 & $2.832 \mathrm{E}+05$ & 350 & 50 & 40 \\
\hline Union Avenue N & concrete & 2.3 & $2.832 \mathrm{E}+05$ & 300 & 30 & 50 \\
\hline Union $\mathrm{N}$ brick inlay & brick & 1.8 & $2.832 \mathrm{E}+06$ & 550 & 40 & 50 \\
\hline Union Avenue N & concrete & 2.3 & $4.786 \mathrm{E}+07$ & 350 & 40 & 40 \\
\hline Wall Avenue & concrete & 2.3 & $4.078 \mathrm{E}+07$ & 300 & 50 & 40 \\
\hline Wall brick inlay & brick & 1.8 & $2.832 \mathrm{E}+06$ & 750 & 60 & 60 \\
\hline Wall Avenue & concrete & 2.3 & $2.832 \mathrm{E}+05$ & 350 & 50 & 40 \\
\hline west sidewalk & brick & 1.8 & $8.155 \mathrm{E}+07$ & 750 & 50 & 50 \\
\hline east sidewalk $\mathrm{N}$ & brick & 1.8 & $7.221 \mathrm{E}+07$ & 650 & 60 & 40 \\
\hline east sidewalk $S$ & brick & 1.8 & $2.039 \mathrm{E}+07$ & 550 & 60 & 60 \\
\hline
\end{tabular}


Table B.5. Computed detector response function $\left([\operatorname{count} / \mathrm{sec}] /\left[1 / \mathrm{cm}^{2} / \mathrm{sec}\right]\right)$ for a $2 \times 4 \times 16$ inch NaI detector with a $1 \mathrm{~mm}$ thick aluminum cover

\begin{tabular}{c|cc|c}
\hline \hline $\begin{array}{c}\text { Energy } \\
(\mathrm{MeV})\end{array}$ & $\begin{array}{c}\text { Count Rate } \\
\text { per unit flux }\end{array}$ & $\begin{array}{c}\text { Energy } \\
(\mathrm{MeV})\end{array}$ & $\begin{array}{c}\text { Count Rate } \\
\text { per unit flux }\end{array}$ \\
\hline 0.0501 & 238.91 & 0.6310 & 234.32 \\
0.0562 & 258.96 & 0.7943 & 223.66 \\
0.0631 & 271.56 & 1.0000 & 213.37 \\
0.0708 & 280.93 & 1.2589 & 202.76 \\
0.0794 & 305.73 & 1.5849 & 192.90 \\
0.0891 & 312.54 & 1.9953 & 184.66 \\
0.1000 & 313.13 & 2.5119 & 178.07 \\
0.1259 & 310.91 & 3.1623 & 172.73 \\
0.1585 & 307.27 & 3.9811 & 169.70 \\
0.1995 & 300.61 & 5.0119 & 168.52 \\
0.2512 & 288.99 & 6.3096 & 169.04 \\
0.3162 & 273.87 & 7.9433 & 171.88 \\
0.3981 & 258.70 & 10.0000 & 175.97 \\
0.5012 & 245.54 & \multicolumn{3}{|}{} \\
\hline \hline & \multicolumn{3}{|l}{} \\
& \multicolumn{3}{|l}{}
\end{tabular}


Table B.6. Relative contribution to the count rate, sorted from highest contributor to lowest

\begin{tabular}{|c|c|c|c|c|c|}
\hline location & $\mathrm{K}-40$ & U-238 & Th-232 & total & cumulative \\
\hline Gay street & $6.48 \mathrm{E}-02$ & $2.18 \mathrm{E}-01$ & $1.56 \mathrm{E}-01$ & 4.39E-01 & 0.4390 \\
\hline Phoenix & $3.19 \mathrm{E}-02$ & 4.27E-02 & $5.08 \mathrm{E}-02$ & $1.25 \mathrm{E}-01$ & 0.5643 \\
\hline Kress Building & $3.98 \mathrm{E}-02$ & 3.72E-02 & 4.43E-02 & $1.21 \mathrm{E}-01$ & 0.6856 \\
\hline east sidewalk & $1.83 \mathrm{E}-02$ & $3.59 \mathrm{E}-02$ & $2.85 \mathrm{E}-02$ & 8.27E-02 & 0.7683 \\
\hline west sidewalk & $1.39 \mathrm{E}-02$ & $1.94 \mathrm{E}-02$ & $2.31 \mathrm{E}-02$ & $5.64 \mathrm{E}-02$ & 0.8247 \\
\hline KUB & $1.47 \mathrm{E}-02$ & $1.33 \mathrm{E}-02$ & $1.91 \mathrm{E}-02$ & 4.71E-02 & 0.8718 \\
\hline Black Roof & $9.23 \mathrm{E}-03$ & 1.19E-02 & $1.70 \mathrm{E}-02$ & $3.82 \mathrm{E}-02$ & 0.9100 \\
\hline Art Market & $1.01 \mathrm{E}-02$ & 7.79E-03 & 9.29E-03 & 2.72E-02 & 0.9371 \\
\hline Woodruff's & $3.27 \mathrm{E}-03$ & $3.32 \mathrm{E}-03$ & $5.93 \mathrm{E}-03$ & $1.25 \mathrm{E}-02$ & 0.9497 \\
\hline Skybox & $2.90 \mathrm{E}-03$ & 4.48E-03 & $3.56 \mathrm{E}-03$ & $1.09 \mathrm{E}-02$ & 0.9606 \\
\hline Baker's & $2.98 \mathrm{E}-03$ & $3.12 \mathrm{E}-03$ & $2.66 \mathrm{E}-03$ & $8.76 \mathrm{E}-03$ & 0.9694 \\
\hline 411 Building & $3.43 \mathrm{E}-03$ & 2.63E-03 & 2.09E-03 & $8.15 \mathrm{E}-03$ & 0.9775 \\
\hline Miller's Building & $1.49 \mathrm{E}-03$ & $2.48 \mathrm{E}-03$ & $2.37 \mathrm{E}-03$ & $6.34 \mathrm{E}-03$ & 0.9839 \\
\hline Mast General Store & $1.15 \mathrm{E}-03$ & $1.44 \mathrm{E}-03$ & $2.06 \mathrm{E}-03$ & $4.64 \mathrm{E}-03$ & 0.9885 \\
\hline Sapphire & $5.10 \mathrm{E}-04$ & 8.66E-04 & $8.25 \mathrm{E}-04$ & $2.20 \mathrm{E}-03$ & 0.9907 \\
\hline Gay street & $3.05 \mathrm{E}-04$ & $5.67 \mathrm{E}-04$ & $9.00 \mathrm{E}-04$ & $1.77 \mathrm{E}-03$ & 0.9925 \\
\hline House & 3.73E-04 & 5.63E-04 & 4.47E-04 & $1.38 \mathrm{E}-03$ & 0.9939 \\
\hline 502 (The Market) & $3.26 \mathrm{E}-04$ & $3.11 \mathrm{E}-04$ & $5.55 \mathrm{E}-04$ & $1.19 \mathrm{E}-03$ & 0.9950 \\
\hline Slomski Taylor & $3.08 \mathrm{E}-04$ & $3.05 \mathrm{E}-04$ & $3.63 \mathrm{E}-04$ & $9.75 \mathrm{E}-04$ & 0.9960 \\
\hline Lerner Lofts & $2.36 \mathrm{E}-04$ & 3.33E-04 & $3.18 \mathrm{E}-04$ & 8.87E-04 & 0.9969 \\
\hline east sidewalk & $1.35 \mathrm{E}-04$ & $2.87 \mathrm{E}-04$ & $3.41 \mathrm{E}-04$ & $7.63 \mathrm{E}-04$ & 0.9977 \\
\hline Gay street & $7.84 \mathrm{E}-05$ & $2.46 \mathrm{E}-04$ & 2.34E-04 & $5.58 \mathrm{E}-04$ & 0.9982 \\
\hline Gay brick inlay & $9.75 \mathrm{E}-05$ & $1.76 \mathrm{E}-04$ & $2.50 \mathrm{E}-04$ & $5.24 \mathrm{E}-04$ & 0.9987 \\
\hline Gay brick inlay $(\mathrm{N})$ & $6.94 \mathrm{E}-05$ & $7.34 \mathrm{E}-05$ & $1.09 \mathrm{E}-04$ & $2.52 \mathrm{E}-04$ & 0.9990 \\
\hline Shorty & $5.21 \mathrm{E}-05$ & 7.31E-05 & $1.05 \mathrm{E}-04$ & $2.30 \mathrm{E}-04$ & 0.9992 \\
\hline Union Avenue N & $2.62 \mathrm{E}-05$ & $5.03 \mathrm{E}-05$ & $6.00 \mathrm{E}-05$ & $1.37 \mathrm{E}-04$ & 0.9994 \\
\hline Alley Cover & $2.26 \mathrm{E}-05$ & $3.75 \mathrm{E}-05$ & $5.38 \mathrm{E}-05$ & $1.14 \mathrm{E}-04$ & 0.9995 \\
\hline Wall Avenue & $1.49 \mathrm{E}-05$ & 4.43E-05 & $4.21 \mathrm{E}-05$ & $1.01 \mathrm{E}-04$ & 0.9996 \\
\hline Gay brick inlay (S) & $1.99 \mathrm{E}-05$ & 4.04E-05 & $4.01 \mathrm{E}-05$ & $1.00 \mathrm{E}-04$ & 0.9997 \\
\hline Union $\mathrm{N}$ brick inlay & $2.14 \mathrm{E}-05$ & 2.89E-05 & 4.29E-05 & $9.32 \mathrm{E}-05$ & 0.9998 \\
\hline Gay street & $1.09 \mathrm{E}-05$ & $3.12 \mathrm{E}-05$ & $3.72 \mathrm{E}-05$ & $7.93 \mathrm{E}-05$ & 0.9999 \\
\hline Wall brick inlay & $1.23 \mathrm{E}-05$ & $1.87 \mathrm{E}-05$ & 2.22E-05 & 5.32E-05 & 0.9999 \\
\hline Union Avenue S & $6.09 \mathrm{E}-06$ & $1.63 \mathrm{E}-05$ & $1.95 \mathrm{E}-05$ & 4.19E-05 & 1.0000 \\
\hline Union $\mathrm{S}$ brick inlay & $7.86 \mathrm{E}-06$ & $1.12 \mathrm{E}-05$ & $1.06 \mathrm{E}-05$ & $2.97 \mathrm{E}-05$ & 1.0000 \\
\hline Union Avenue N & $1.59 \mathrm{E}-06$ & 2.89E-06 & $5.74 \mathrm{E}-06$ & $1.02 \mathrm{E}-05$ & 1.0000 \\
\hline Wall Avenue & $9.48 \mathrm{E}-07$ & $2.53 \mathrm{E}-06$ & $2.41 \mathrm{E}-06$ & 5.89E-06 & 1.0000 \\
\hline Union Avenue S & $6.57 \mathrm{E}-07$ & $1.70 \mathrm{E}-06$ & $1.62 \mathrm{E}-06$ & $3.98 \mathrm{E}-06$ & 1.0000 \\
\hline Total & 0.2206 & 0.4078 & 0.3716 & 1.0000 & \\
\hline
\end{tabular}




\section{References}

[1] Gauld, Ian C., Georgeta Radulescu, Germina Ilas, Brian D. Murphy, Mark L. Williams, and Dorothea Wiarda. 2011. "Isotopic Depletion and Decay Methods and Analysis Capabilities in SCALE," Nuclear Technology 174, No. 2, 169-195.

[2] Hamilton, Ian Scott. 2009. "Chapter 3. Industry-Specific Description of the NORM Source Term." Naturally occurring radioactive materials (NORM) and technologically enhanced NORM (TENORM). Health Physics Society Professional Development School (2009) Ed. by P. Andrew Karam and Brian J. Vetter.

[3] Kouzes, Richard T. 2009. "Chapter 12. NORM Impact on Interdiction at Borders.” Naturally occurring radioactive materials (NORM) and technologically enhanced NORM (TENORM). Health Physics Society Professional Development School (2009) Ed. by P. Andrew Karam and Brian J. Vetter.

[4] Gardner, Robin P. and Avneet Sood. 2004. "A Monte Carlo simulation approach for generating NaI detector response function (DRFs) that accounts for non-linearity and variable flat continua." Nuclear Instruments and Methods in Physics Research B 213, 87-99.

[5] X-5 Monte Carlo Team. 2003. MCNP-A General Monte Carlo N-Particle Transport Code, Version 5. Volume I: Overview and Theory. LA-UR-03-1987. Los Alamos National Laboratory, Los Alamos, NM.

[6] Peplow, Douglas E. 2011. "Monte Carlo Shielding Analysis Capabilities with MAVRIC." Nuclear Technology 174, No. 2, 289-313.

[7] Evans, T. M., A. S. Stafford, R. N. Slaybaugh, and K. T. Clarno. 2010. "Denovo: A New Three-Dimensional Parallel Discrete Ordinates Code in SCALE.” Nuclear Technology 171, No. 2, 171-200.

[8] Wagner, John C., Douglas E. Peplow, and Scott W. Mosher. 2014. "FW-CADIS Method for Global and Semi-Global Variance Reduction of Monte Carlo Radiation Transport Calculations." Nuclear Science and Engineering 176, No. 1, 37-57. 